Aus der Abteilung Kardiologie und Intensivmedizin

(Chefarzt: Prof. Dr. med. K.H. Scholz)

des St. Bernward Krankenhauses in Hildesheim

\title{
Verbesserung der Herzinfarktversorgung durch standardisierte Datenerfassung und systematische Ergebnis-Rückkopplung
}

Eine prospektive Untersuchung aus dem

Herzinfarktnetz Hildesheim-Leinebergland

\author{
INAUGURAL -DISSERTATION
}

zur Erlangung des Doktorgrades

der Medizinischen Fakultät

der Georg-August-Universität zu Göttingen

vorgelegt von

Dorothe Ahlersmann

aus

Georgsmarienhütte

Göttingen 2010 
D e k a n:

I. Berichterstatter:

II. Berichterstatter:

Tag der mündlichen Prüfung:
Prof. Dr. med. C. Frömmel

Prof. Dr. med. K.H. Scholz

Priv.-Doz. Dr. med. Eich

17.11.2010 


\section{INHALTSVERZEICHNIS}

\section{EINLEITUNG}

1.1. ST-Streckenhebungsinfarkt

1.1.1. Epidemiologie

1.1.2. Definition, Pathogenese und Diagnostik 2

1.1.3. Leitlinienkonforme Therapie 4

1.1.4. Zeitfaktor und strukturierte Versorgungskonzepte 6

1.1.5. Aktuelle Umsetzung der Leitlinien $\quad 8$

1.2. Herzinfarktnetz Hildesheim-Leinebergland 10

\begin{tabular}{lr} 
2. FRAGESTELLUNG & 15 \\
\hline
\end{tabular}

3. PATIENTEN UND METHODEN

3.1. Patientenkollektiv 16

3.1.1.Nicht in die Auswertung eingeschlossene Patienten $\quad 17$

$\begin{array}{ll}\text { 3.1.2. Patientengruppen } & 17\end{array}$

3.2. Datenerfassung 18

3.3. Zeiterfassung 20

3.4. Basischarakteristika 23

3.5. Projektablauf 25

3.6. Chronologische Projektdurchführung 27

3.7. Qualitätsmanagement-Interventionen 27

3.8. Kernqualitätsindikatoren 28

3.9. Verstorbene Patienten 28

3.10. Statistische Auswertung 29

4. ERGEBNISSE

\begin{tabular}{ll}
\hline 4.1. Patienten & 30
\end{tabular}

4.1.1. Nicht in die Auswertung eingeschlossene Patienten 30

4.1.2. Eingeschlossene Patienten 31

4.1.3. Basischarakteristika $\quad 32$

4.1.4. Herkunft der Patienten 34

4.2. Zeitintervalle 40

4.2.1. Gesamtgruppe 40

4.2.2. Patienten mit Primärtransport 45

4.2.3. Patienten mit Sekundärtransport $\quad 48$

$\begin{array}{ll}\text { 4.2.4. Patienten mit Selbsteinweisung } & 49\end{array}$

4.3. Funk-EKG und Umgehung der Notaufnahme (,Bypassing“) 50

4.4. Verstorbene Patienten 53

5. DISKUSSION $\quad \mathbf{5 4}$

5.1. Prozessoptimierung durch standardisiertes Qualitätsmanagement 54

5.2. Bedeutung von Funk-EKG und Umgehung der Notaufnahme (Bypassing) 59

5.3. Limitationen $\quad 63$

5.4. Ausblick 64 
$\begin{array}{ll}\text { 7.1. Abbildungsverzeichnis } & 69\end{array}$

$\begin{array}{ll}\text { 7.2. Tabellenverzeichnis } & 70\end{array}$

$\begin{array}{ll}\text { 7.3. Abkürzungsverzeichnis } & 71\end{array}$

7.4. Dokumentationsbögen $\quad 73$

7.4.1. Web-basierter Dokumentationsbogen 10/07 73

7.4.2. Weiterentwickelter Web-basierter Dokumentationsbogen 06/09 75

8. LITERATURVERZEICHNIS $\quad 77$

9. VERÖFFENTLICHUNGEN 


\section{Einleitung}

\subsection{ST-Streckenhebungsinfarkt (STEMI)}

\subsubsection{Epidemiologie}

Die kardiovaskulären Erkrankungen stehen nach wie vor an erster Stelle der Todesursachenstatistik in der Bundesrepublik Deutschland und den westlichen Industriestaaten. Nach aktuellen Erhebungen des Statistischen Bundesamtes wird bei nahezu jedem zweiten Verstorbenen der Tod durch eine Erkrankung des Herzkreislaufsystems ausgelöst. Der akute Myokardinfarkt führt dabei die Einzeltodesursachenstatistik an (Statistisches Bundesamt 2006). Pro Jahr erleiden in Deutschland ca. 300.000 Menschen einen akuten Myokardinfarkt. Die Gesamtsterblichkeit beträgt ca. 25\%, wovon ca. zwei Drittel auf die prähospitale Phase entfallen, während die Mortalität im Krankenhaus in den letzten Jahren deutlich gesenkt werden konnte (de Vreede et al. 1991, Fox et al. 2000, The GRACE Investigators 2001, Tunstall-Pedoe et al. 1999, Zeymer 2005).

Von den nationalen und internationalen kardiologischen Fachgesellschaften (DGK, ESC, ACC/AHA) wurden Leitlinien zur Behandlung des STEMI verfasst, in denen die diagnostischen und therapeutischen Möglichkeiten evaluiert werden. Durch entsprechende standardisierte Empfehlungen soll die Behandlung effizienter gestaltet und konsekutiv die Mortalität gesenkt werden (Antman et al. 2004, Hamm et al. 2004, Van de Werf et al. 2003). 


\subsubsection{Definition, Pathogenese und Diagnostik}

Definitionsgemäß wird unter einem Myokardinfarkt der Tod von Kardiomyozyten auf dem Boden einer prolongierten Ischämie verstanden. Dabei finden klinische, elektrokardiographische, biochemische und pathologische Charakteristika Anwendung.

Kürzlich wurde die Definition des akuten Myokardinfarkts unter besonderer Berücksichtigung von biochemischen in Verbindung mit klinischen Kriterien erweitert. Die klinische Behandlungsstrategie richtet sich jedoch nach elektrokardiographischen Aspekten. So wird unter dem Begriff des akuten Koronarsyndroms zwischen ST-Streckenhebungsinfarkt (STEMI), Nicht-ST-Streckenhebungsinfarkt (NSTEMI) und instabiler Angina pectoris unterschieden, zwischen denen ein fließender Übergang besteht (The Joint European Society of Cardiology 2000).

$\mathrm{Zu}$ einem STEMI kommt es, wenn die Koronardurchblutung abrupt durch einen thrombotischen Verschluss einer Herzkranzarterie unterbrochen wird. In der überwiegenden Zahl der Fälle tritt ein Infarkt auf, sobald eine atherosklerotische Plaque einreißt, platzt oder ulzeriert und sich ein wandständiger Thrombus an der Stelle der Ruptur bildet, der zu einer Koronararterienokklusion führt. Bereits 1977 wurde anhand von tierexperimentellen Untersuchungen an Hunden nachgewiesen, dass die Infarktgrößenentwicklung eindeutig von der Dauer der Okklusion des verschlossenen Herzkranzgefäßes abhängig ist. Der durch einen kompletten Koronararterienverschluss verursachte myokardiale Zelluntergang beginnt nach ca. 15-30 Minuten, schreitet zeitabhängig vom Endokard bis Epikard voran (wave-front phenomenon) und ist in aller Regel nach 6 Stunden komplett abgeschlossen (Reimer et al. 1977).

Die Diagnose wird in erster Linie anhand von klinischem Befund und typischen EKGKriterien gestellt. Leitsymptom ist der retrosternal betonte Brustschmerz, der häufig mit Ausstrahlung in Nacken, Hals, Kiefer, Arme oder Oberbauch verbunden ist. Daneben wird die Symptomatik in vielen Fällen von vegetativen Zeichen wie Dyspnoe, Schweißausbruch, Übelkeit und Todesangst begleitet. Insbesondere bei älteren Patienten, Frauen und Diabetikern werden gehäuft atypische Beschwerden beobachtet. Charakteristisch für den STEMI ist eine länger anhaltende (>20 min) und nitrorefraktäre Schmerzsymptomatik. 
Das Schlüsselinstrument zur Abgrenzung des STEMI von anderen Formen des akuten Koronarsyndroms und gleichzeitig die wichtigste Grundlage aller weiteren Entscheidungsprozesse stellt das Zwölf-Kanal-EKG dar, welches nach den Leitlinien vom Notarzt prästationär bzw. spätestens innerhalb von 10 min nach Ankunft im Krankenhaus zu schreiben und sofort von einem qualifizierten Arzt zu beurteilen ist (Hamm et al. 2004).

Die Indikationsstellung zur Reperfusionstherapie basiert auf den folgenden EKG-Kriterien:

$>$ ST-Streckenhebung von $\geq 0,1 \mathrm{mV}$ in mindestens zwei zusammenhängenden Extremitätenableitungen oder

$>0,2 \mathrm{mV}$ in mindestens zwei zusammenhängenden Brustwandableitungen oder

Linksschenkelblock mit infarkttypischer Symptomatik.

Daneben ist ergänzend bei Verdacht auf einen inferioren Infarkt die Ableitung V4 rechts zur Abschätzung einer rechtsventrikulären Infarktbeteiligung $\mathrm{zu}$ dokumentieren. Durch die zusätzlichen Brustwandableitungen V7-V9 kann die Diagnose eines posterioren Infarkts verifiziert werden. Insbesondere sind auch ST-Streckensenkungen in den gegensinnigen Ableitungen zu berücksichtigen. Das Ausmaß der ST-Streckenhebungen und die Anzahl der betroffenen Ableitungen korreliert mit der Größe der vom Untergang bedrohten Muskelmasse (Hamm et al. 2004, Van de Werf et al. 2003).

Bei Patienten mit elektrokardiographisch nachgewiesenem STEMI mit typischer Klinik ist die Bestimmung der spezifischen biochemischen Marker (Myoglobin, Troponin T, Troponin I, CK-MB) wegen eines möglichen fehlenden Anstiegs innerhalb der ersten 2 Stunden nach Infarktbeginn und wegen der zeitlichen Dringlichkeit der Reperfusionsmaßnahmen ohne Bedeutung. Im Verlauf des Infarktes können die Maximalwerte von CK, CK-MB und Troponin zur Abschätzung der Infarktgröße hilfreich sein, auch lässt sich im Verlauf ein Rezidiv oder Zweitinfarkt erkennen.

Andere diagnostische Maßnahmen sind in der Routinediagnostik des Myokardinfarkts nicht erforderlich. Bei Diskrepanz zwischen elektrokardiographischem und klinischem Befund kann die zweidimensionale Echokardiographie zur Entscheidungsfindung hinzugezogen werden (Hamm et al. 2004). 


\subsubsection{Leitlinienkonforme Therapie}

Vorrangiges Ziel in der Therapie des STEMI ist die Reperfusion des verschlossenen Herzkranzgefäßes. Hierfür stehen unter stationären Bedingungen prinzipiell zwei Therapiekonzepte zur Verfügung: Primäre perkutane Koronarintervention (PCI) oder Fibrinolyse, oder die Kombination aus beiden.

Innerhalb der ersten 3 Stunden nach Symptombeginn ist die Thrombolyse eine mögliche Alternative zur primären PCI. Sie sollte insbesondere dann zum Einsatz kommen, wenn aufgrund ungünstiger Transportbedingungen und mangelnder Transportkapazitäten die zu erwartenden Zeitverluste bis zum Beginn einer PCI 90 min überschreiten. Bei Patienten, deren Symptombeginn bereits länger als 3 Stunden zurückliegt, ist vorrangig die PCI indiziert (Bonnefoy et al. 2002, Zahn et al. 2001). Darüber hinaus ist die primäre PCI die einzige Option zur Revaskularisation, wenn Kontraindikationen zur Lyse bestehen (Zahn et al. 1999) sowie bei Patienten im kardiogenen Schock auch jenseits der 12-Stunden-Grenze (Hochman et al. 1999, Urban et al. 1999).

In den letzten Jahren konnte durch zahlreiche randomisierte Studien der Vorteil der primären PCI als routinemäßige Reperfusionsstrategie bezüglich der Reduktion der Infarktsterblichkeit sowohl im Früh- als auch im Langzeitverlauf im Vergleich zur Fibrinolyse belegt werden (Andersen et al. 2003, Bonnefoy et al. 2002, Gibbons et al. 1993, Grines et al. 1993, Keeley et al. 2003, Steg et al. 2003, Widimsky et al. 2003, Zijlstra et al. 1993) (Evidenzgrad I-A). Aus der Metaanalyse der verfügbaren Daten geht hervor, dass durch die PCI im Vergleich zur Fibrinolyse eine Reduktion der Infarktsterblichkeit um 25\% erzielt wird (Grines et al. 1993, Keeley et al. 2003). Zusätzlich ist bei PCI das Schlaganfallrisiko durch Vermeidung von Hirnblutungen gegenüber der Lysetherapie um absolut $1 \%$ und das Reinfarktrisiko um mehr als die Hälfte reduziert (Keeley et al. 2003). Darüber hinaus wurde gezeigt, dass diese positiven Effekte der primären PCI im Langzeitverlauf erhalten bleiben (Nunn et al. 1999, Zijlstra et al. 1999). Dabei war der Vorteil der PCI im Vergleich zur Fibrinolyse unabhängig davon, ob die Patienten im aufnehmenden Krankenhaus versorgt werden konnten oder zur Durchführung einer PCI in eine Interventionsklinik transferiert werden mussten (Dalby et al. 2003, Keeley et al. 2003, Widimsky et al 2003). 
Die akute Bypasschirurgie besitzt aufgrund der beträchtlichen Zeitverzögerung bis zum Beginn der Operation und der hohen Komplikationsrate von Akuteingriffen im Infarkt als routinemäßige Alternative keinen Stellenwert. Ein akutes operatives Vorgehen kann im Fall einer erfolglosen PCI mit persistierendem Gefäßverschluss und anhaltender hämodynamischer Instabilität, einer für eine kathetertechnische Intervention ungeeigneten Koronarmorphologie bzw. bei schweren Infarktkomplikationen wie Septumruptur oder Papillarmuskelabriß in Betracht gezogen werden (Evidenzgrad I-C) (Hamm et al. 2004).

Die schnelle und sichere Diagnosestellung mittels Zwölf-Kanal-EKG mit sofort nachfolgender Ankündigung in einem Interventionszentrum mit 24h Bereitschaft und die unmittelbare Durchführung einer primären PCI mit adjuvanter medikamentöser Therapie stellt den idealen Behandlungsablauf für einen Patienten mit STEMI dar. Ein solches Vorgehen ist wahrscheinlich von entscheidender Bedeutung für die Gesamtprognose. 


\subsubsection{Zeitfaktor und strukturierte Versorgungskonzepte}

Gemäß dem Pathomechanismus der Infarktentstehung und -ausbreitung begrenzt eine frühzeitig eingeleitete Reperfusionstherapie die Infarktgröße und kann zu einer Reduktion der Sterblichkeit führen. In mehreren Untersuchungen konnte gezeigt werden, dass kürzere doorto-balloon-Zeiten (Ankunft Interventionsklinik bis 1. Balloninflation im Infarktgefäß) mit einer geringeren Sterblichkeit einhergehen (Berger et al. 1999, Cannon et al. 2000). Daten aus dem groß angelegten amerikanischen NRMI-Register (National Registry of Myocardial Infarction) belegen, dass sich bei door-to-balloon-Zeiten zwischen 90 und 150 Minuten die Krankenhaussterblichkeit pro 15-min-Zeitverlust um 6,3 Todesfälle je 1000 behandelte Patienten erhöht (Mc Namara et al. 2006a). Die zeitliche Abhängigkeit unterstreicht die Bedeutung einer raschen Therapieeinleitung bei der Versorgung von Patienten mit STEMI. Diesem Zeitfaktor wird in den nationalen und internationalen Leitlinien Rechnung getragen. Gemäß den Vorgaben der Deutschen Gesellschaft für Kardiologie gilt, dass die door-toballoon-Zeit bei einer primären PCI ohne Ankündigung 60 min (mit Ankündigung 30 min) nicht überschreiten sollte. Die Zielvorgabe für die contact-to-balloon-Zeit (Intervall Erstkontakt mit dem Rettungssystem bis zur 1. Balloninflation) liegt bei weniger als $120 \mathrm{~min}$ (Hamm et al. 2004). Im internationalen Vergleich variieren die empfohlenen Zeitvorgaben. So sollte entsprechend der Europäischen Gesellschaft für Kardiologie die contact-to-balloon-Zeit nicht mehr als 90 min betragen (Van de Werf et al. 2003), wohingegen die amerikanischen kardiologischen Fachgesellschaften eine maximale door-to-balloon-Zeit von $90 \mathrm{~min}$ empfehlen (Antman et al. 2004). In einem Update aus 2008 wurden diese Empfehlungen dahingehend modifiziert, dass die geforderte door-to-balloon-Zeit von < 90 min für die Gruppe der Selbstvorsteller gilt. Bei Patienten mit Sekundär- und Primärtransport wird jetzt eine Zeitdauer von < 90 min für Ankunft des medizinischen Systems am Einsatzort bis Balloninflation gefordert (contact-to-balloon). Erstmals wird der Begriff der totalen Ischämiezeit eingeführt, der eine empfohlene Zeitdauer von < 120 min von Symptombeginn bis Reperfusion beschreibt (Antman et al. 2008).

Zur Gewährleistung einer leitlinienkonformen Infarkttherapie ist es notwendig, Strukturen zu schaffen, die unter Berücksichtigung lokaler Gegebenheiten die Basis für einheitliche Prozessabläufe bieten. 
Um alle Patienten mit STEMI einer notfallmäßigen Akut-PCI zuführen zu können, wird von den Leitlinien ein Herzkatheterlabor mit 24-Stunden-Bereitschaft des Katheterpersonals sowie erfahrene Interventionskardiologen mit mindestens 40 Infarkt-Interventionen/Jahr gefordert.

Das Katheterteam sollte nach rascher und möglichst prähospitaler Diagnosestellung unmittelbar einsatzbereit sein. Darüber hinaus wird eine gute intrahospitale Logistik mit kurzen Zeitintervallen zwischen stationärer Aufnahme des Patienten bis zur Katheterintervention gefordert.

Insgesamt handelt es sich bei der Rettungs-/Diagnose-, Alarmierungs- und Therapiekette um einen komplexen und mehrdimensionalen Prozessablauf mit unterschiedlichen Schnittstellen. An der Akutversorgung eines Patienten mit STEMI sind von der Alarmierung bis zur Katheterintervention in der Regel zehn bis fünfzehn Personen aus unterschiedlichen Berufsgruppen, Abteilungen und ggfs. auch Krankenhäusern in nur selten identischer Konstellation beteiligt, die unter großem Zeitdruck in Regeldienst oder Rufbereitschaft kooperieren müssen. Erschwerend kommt hinzu, dass in Deutschland nur bei weniger als 20\% aller derjenigen Krankenhäuser, die Herzinfarktpatienten versorgen, die Möglichkeit zur primären PCI gegeben ist (Hamm et al. 2004). Die kardiologischen Fachgesellschaften propagieren daher die Bildung von Herzinfarktnetzen. Anhand von strukturierten Versorgungskonzepten soll eine optimale Zusammenarbeit zwischen Rettungsdienst, nichtinvasiv tätigen Krankenhäusern und Kliniken mit Herzkatheterlabor gewährleistet werden. Hierzu ist ein klares und genau definiertes Ablaufprotokoll unabdingbar, das einen festen Algorithmus mit unkompliziertem Meldesystem unter Einbeziehung eines direkt verantwortlichen Ansprechpartners mit absoluter Sach- und Entscheidungskompetenz in der Interventionsklinik vorsieht. Eine weitere Voraussetzung zur raschen Diagnosestellung ist die frühzeitige und prähospitale Anfertigung eines 12-Kanal-EKG. Erst hierdurch ist die Forderung der Leitlinien, die nächstgelegene Nicht-Interventionsklinik zu umgehen, und die Interventionsklinik nach Vorabkontakt durch den primär versorgenden Notarzt direkt anzufahren (Bypassing der Nicht-Interventionsklinik), überhaupt realisierbar (Hamm et al. 2004). Ebenso wird durch die prähospitale Diagnosestellung mittels eines 12-Kanal-EKG eine systematische Umgehung der Notaufnahme der Interventionsklinik mit Direktübergabe des Patienten durch den Notarzt an den Interventionskardiologen (Bypassing der Notaufnahme) ermöglicht. Wird zusätzlich die Option der Telemetrie genutzt, wird eine sichere Basis für die Alarmierung und eine Vermeidung von Fehleinsätzen des Katheterteams gewährleistet. 


\subsubsection{Aktuelle Umsetzung der Leitlinien}

Anhand von umfangreichen Registerdaten aus prospektiven multizentrischen Studien wurde die Umsetzung einer leitliniengerechten Therapie im klinischen Alltag untersucht (Rustige et al. 1997, Wagner et al. 1999, Wienbergen et al. 2003, Zahn et al. 2000). Eine frühzeitige Reperfusionstherapie wurde in Deutschland im Jahr 2000-2002 mit steigender Tendenz bei zuletzt etwa $60 \%$ der Patienten mit STEMI durchgeführt, wobei der Anteil der Patienten mit primärer PCI über die Jahre zugenommen hat. Dabei wurde in Krankenhäusern mit Herzkatheterlabor häufiger eine primäre PCI und in Krankenhäusern ohne Herzkatheterlabor eher eine Thrombolyse durchgeführt (Zeymer et al. 2005). Eine unbegründet vorenthaltene Reperfusionstherapie ist mit einer hohen Mortalität assoziiert. So betrug die Krankenhaussterblichkeit bei diesen Patienten etwa 20\%, die 1-Jahres-Sterblichkeit $40 \%$. Nach den Daten der Herzinfarktregister betrug die door-to-balloon-Zeit (Klinikankunft bis 1. Balloninflation) im Mittel 75 min (Wienbergen et als. 2005). Das Zeitintervall zwischen Symptombeginn und Krankenhausaufnahme (Prähospitalzeit) verlängerte sich in den vergangenen Jahren trotz intensiver Bemühungen auf im Mittel 190 min. Insgesamt hat der Anteil der Patienten mit prognostisch wirksamer leitliniengerechter STEMI-Behandlung (mit Fokussierung auf die adjuvante medikamentöse Akuttherapie) im Vergleich $\mathrm{zu}$ früheren Registerdaten mit vergleichbarem Patientenkollektiv zugenommen (Wienbergen et al. 2005). Eine Beobachtungsstudie aus dem PCI-Register der ALKK (Arbeitsgemeinschaft Leitender Kardiologischer Krankenhausärzte) ermittelte in einem Kollektiv von 4815 Patienten aus 80 Zentren zwischen 1994 und 2002 eine mittlere „door-to-angiography“-Zeit (Ankunft Klinik bis Punktion) von 83 min (Median $50 \mathrm{~min}$ ), wobei bei 2/3 der Patienten die Punktion innerhalb von 60 min erfolgte. Zur Vergleichbarkeit mit dem ansonsten üblichen Terminus door-to-balloon-Zeit wird von den Autoren eine Differenz von 10-15 min (Punktion bis Balloninflation) unterstellt (Zahn et al. 2006), die der „door-to-angiography“-Zeit noch hinzugerechnet werden müsste.

In den Vereinigten Staaten von Amerika sehen sich die Kliniken mittlerweile einem noch größeren Druck ausgesetzt, die Reperfusionszeiten zu verkürzen, nachdem die door-toballoon-Zeiten in dem Bestreben um eine Verbesserung der Versorgungsqualität in der STEMI-Behandlung als einer der Kernqualitätsindikatoren der Centers for Medicare und Medicaid Services (CMS) regelmäßig veröffentlicht werden (Specification Manual for National Hopsital Quality Measures, Version 2b). 
Daten aus dem amerikanischen National Registry of Myocardial Infarction (NRMI) zeigen, dass zwischen 1999 und 2002 weniger als die Hälfte der Patienten mit STEMI innerhalb der von den amerikanischen Leitlinien geforderten door-to-balloon-Zeit von 90 Minuten behandelt wurden (McNamara et al. 2006 b). Kürzlich wurden von Bradley et al. in einer an 365 US-Krankenhäusern durchgeführten Querschnittstudie mittels Fragebogen 6 aus 28 Strategien identifiziert, die mit einer Reduktion der Reperfusionszeiten assoziiert waren. Die identifizierten Strategien waren bei multivariater Analyse jeweils mit einem mittleren Zeitgewinn zwischen 8 und 15 min verbunden. Dennoch wurden diese Strategien nur von einer Minderheit der untersuchten Kliniken umgesetzt, so dass der Mittelwert der Mediane der door-to-balloon-Zeiten in diesem Kollektiv US-amerikanischer Spitzen-Krankenhäusern 100,4 $\pm 23,5$ min betrug (Bradley et al. 2006 a).

Insgesamt bleibt festzuhalten, dass trotz intensiver Bemühungen eine Umsetzung der Leitlinien mit Einhalten der geforderten maximalen Behandlungszeiten sowohl national als auch international nur unzureichend gelingt. Um die Prozessabläufe nachhaltig zu optimieren, erscheint eine systematische Qualitätskontrolle mit dem Ziel einer kontinuierlichen Qualitätsverbesserung unabdingbar. 


\subsection{Herzinfarktnetz Hildesheim-Leinebergland}

Um die primäre PCI als routinemäßige Reperfusionsstrategie innerhalb der geforderten Zeitvorgaben flächendeckend anbieten zu können, fordert die Deutsche Gesellschaft für Kardiologie in ihren aktuellen Leitlinien die Kooperation von nicht-invasiv tätigen Krankenhäusern mit Interventionskliniken im Rahmen von Herzinfarktnetzen mit Etablierung von systematischen Behandlungsstrategien (Hamm et al. 2004). Innerhalb der vergangenen Jahre wurden zahlreiche Herzinfarktnetze teilweise im Rahmen von vorbestehenden Herzinfarktregistern in städtischen Bereichen als auch insbesondere in ländlichen Gebieten gegründet (z.B. Herzinfarktverbund Essen (Bruder et al. 2007), Drip\&Ship-Netzwerk Rostock (Schneider et al. 2007)).

Die aus den Metaanalysen abgeleiteten Vorteile der primären PCI gelten letztendlich nur für erfahrene PCI-Zentren. In mehreren Studien konnte belegt werden, dass die Krankenhausmortalität bei Patienten mit STEMI, die sich einer primären PCI unterzogen, niedriger ist, wenn sie von erfahrenen Untersuchern an einem „high-volume-Zentrum“ durchgeführt wurde (Canto et al 2000, Vakili et al. 2001). Die deutschen Leitlinien empfehlen daher eine Mindestanzahl von 40 Infarkt-Interventionen pro Jahr an Interventionskliniken (Hamm et al 2004). Anhand der Vorgaben der American Heart Association gilt ein Untersucher mit jährlich mehr als 74 Katheterinterventionen als erfahren. Ein Herzkatheterlabor sollte mindestens 200 Katheterinterventionen/Jahr mit anteilsmäßig mindestens 36 primären PCI bei STEMI durchführen. Gleichfalls wurde anfangs eine Kardiochirurgische Abteilung vor Ort gefordert (Antman et al. 2004). Allerdings konnten weitere Studien belegen, dass das klinische outcome der Patienten mit oder ohne Kardiochirurgie vor Ort nicht voneinander abweicht (Ting et al. 2006, Wharton et al. 2004).

Die Abteilung für Invasive Kardiologie am St. Bernward Krankenhaus Hildesheim wurde mit Inbetriebnahme des Herzkatheterlabors im November 2001 neu gegründet und hat sich von Anfang an auf die Behandlung des Akuten Koronarsyndroms und Notfalleingriffe bei akutem Myokardinfarkt spezialisiert. Von vornherein stand eine zweite Herzkatheterlaboreinheit zur parallelen Behandlung von Notfällen zur Verfügung. Gleichfalls wurde seit dem 01.01.2002 ein 24-Stunden-Rufbereitschaftsdienst sowohl für einen erfahrenen Interventionskardiologen als auch für das Assistenzpersonal geschaffen, diese Personen sind innerhalb von 20 Minuten im Herzkatheterlabor einsatzfähig. 
Im Jahr 2005 wurden am St. Bernward Krankenhaus 396 Katheterinterventionen (PCI) vorgenommen, darunter befanden sich 215 PCI bei Patienten mit akutem Myokardinfarkt (STEMI und NSTEMI). Bei erforderlichen kardiochirurgischen Eingriffen werden die Patienten in die 90 km entfernte Abteilung für Thorax-/Herz- und Gefäßchirurgie der GeorgAugust-Universität Göttingen verlegt.

Seit Gründung der Abteilung für Invasive Kardiologie am St. Bernward Krankenhaus in Hildesheim bestand bei der Behandlung des akuten Myokardinfarkts von Beginn an eine enge Zusammenarbeit mit den benachbarten regionalen Nicht-Interventionskliniken in Alfeld und Gronau, aus der sich in den Folgejahren ein bewährter und gut funktionierender Herzinfarktverbund entwickelte, der letztendlich in einem strukturierten Herzinfarktnetz mit festgelegten Organisationsabläufen und Bestimmung von Therapiepfaden mündete. Seit Sommer 2005 sind alle 4 Notarzteinsatzfahrzeuge (NEF) der Region Hildesheim, welche jeweils bei den einzelnen Kliniken stationiert sind, mit telemetriefähigen 12-Kanal-EKGGeräten ausgestattet.

Der Landkreis Hildesheim umfasst eine Fläche von etwa $1.200 \mathrm{~km}^{2}$ mit einer NordSüdausdehnung von $45 \mathrm{~km}$ und eine West-Ostausdehnung von $49 \mathrm{~km}$. Die Einwohnerzahl beträgt 290.643 (Stand 31.12.2005). Das St. Bernward Krankenhaus (524 Betten) ist im Kern der Stadt Hildesheim angesiedelt. Das 25,7 km entfernte kooperierende Regionskrankenhaus in Alfeld (204 Betten) ist im südlichen Landkreis in bergiger Landschaft gelegen, währenddessen sich das Krankenhaus in Gronau (137 Betten) im westlichen Landkreis mit einer Entfernung von 20,8 km zur Interventionsklinik befindet. Innerhalb der Region beschränkt sich dadurch die maximale Transportdauer zur Interventionsklinik auf etwa 50 Minuten. In der Stadt Hildesheim existiert noch ein weiteres interventionell tätiges Krankenhaus in privater Trägerschaft, welches nicht an dem Herzinfarktnetz HildesheimLeinebergland partizipiert (Abbildung 1).

Das Einzugsgebiet des regionalen Rettungsdienstes umfasst auch mitunter Regionen, die sich außerhalb aber angrenzend an den Landkreis Hildesheim befinden. In Einzelfällen werden Patienten aus diesen Regionen aufgrund des kürzeren Anfahrtsweges in das St. Bernward Krankenhaus Hildesheim transportiert. Die zum Einsatz kommenden Notarzteinsatzfahrzeuge aus dem zuständigen Landkreis sind weder am Herzinfarktnetz Hildesheim-Leinebergland beteiligt noch mit einem telemetriefähigen 12-Kanal-EKG-Gerät ausgestattet. 


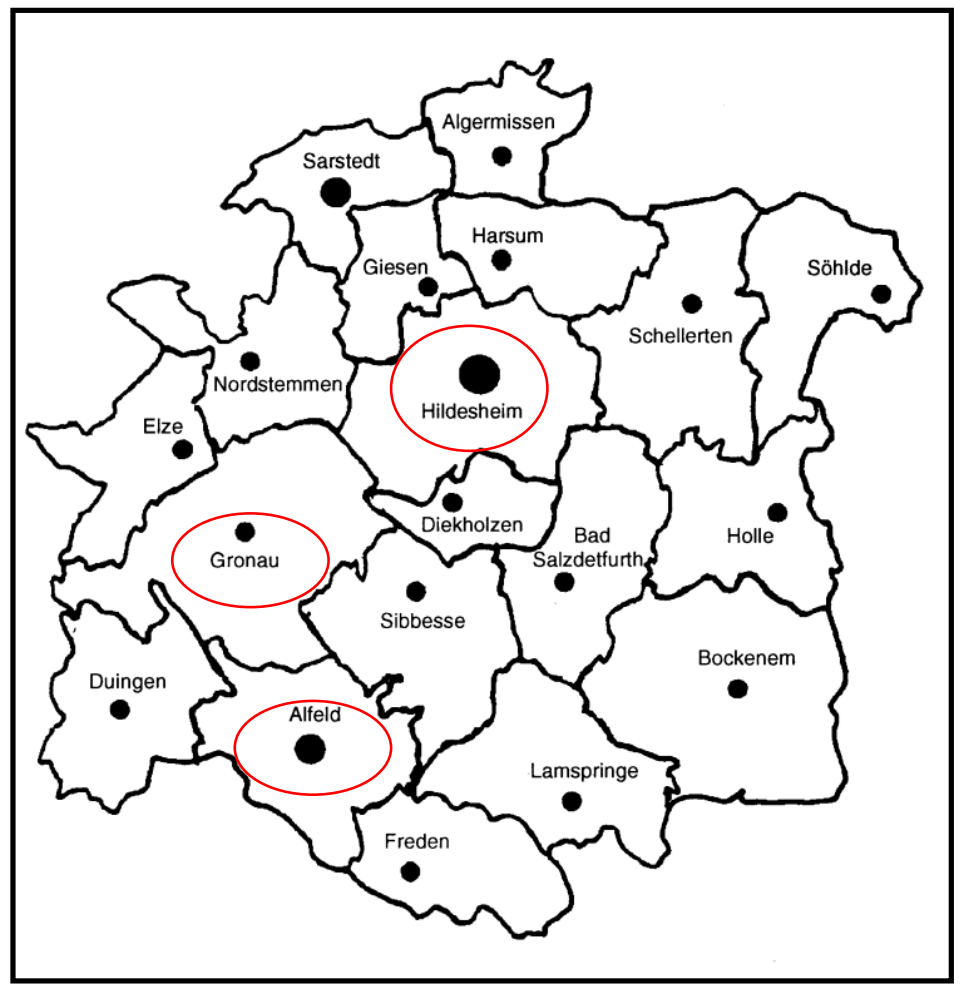

Abbildung 1: Landkreis Hildesheim mit geographischer Lage der am Infarktnetz Hildesheim-Leinebergland beteiligten Kliniken

Die drei entscheidenden Zielpunkte innerhalb des Herzinfarktnetzes HildesheimLeinebergland sind die rasche Identifizierung von Patienten mit STEMI (Diagnosestellung), die unmittelbare Alarmierung des Katheterteams sowie der schnelle und direkte Transport des Patienten in das Herzkatheterlabor. Bei Vorliegen eines typischen klinischen Beschwerdebildes erfolgt anhand eines festgelegten Algorithmus routinemäßig bereits am Einsatzort durch das Team der Rettungsassistenten und des Notarztes die Ableitung eines 12Kanal-EKG (Abbildung 2). Über eine im Gerät vorprogrammierte Faxnummer wird das EKG per Knopfdruck direkt auf die Internistisch-Kardiologische Intensivstation des St. Bernward Krankenhauses übermittelt (Abbildung 3). Parallel dazu wird ein telefonischer Kontakt zum diensthabenden Arzt der Intensivstation hergestellt, der bezüglich des weiteren Vorgehens als zentraler Ansprechpartner und Entscheidungsträger fungiert. Bei Bestätigung der Diagnose eines STEMI erfolgt der unmittelbare Transport in die Interventionsklinik, wobei die möglicherweise näher am Einsatzort gelegene Nicht-Interventionsklinik explizit umgangen wird (Bypassing der Nicht-Interventionsklinik). 


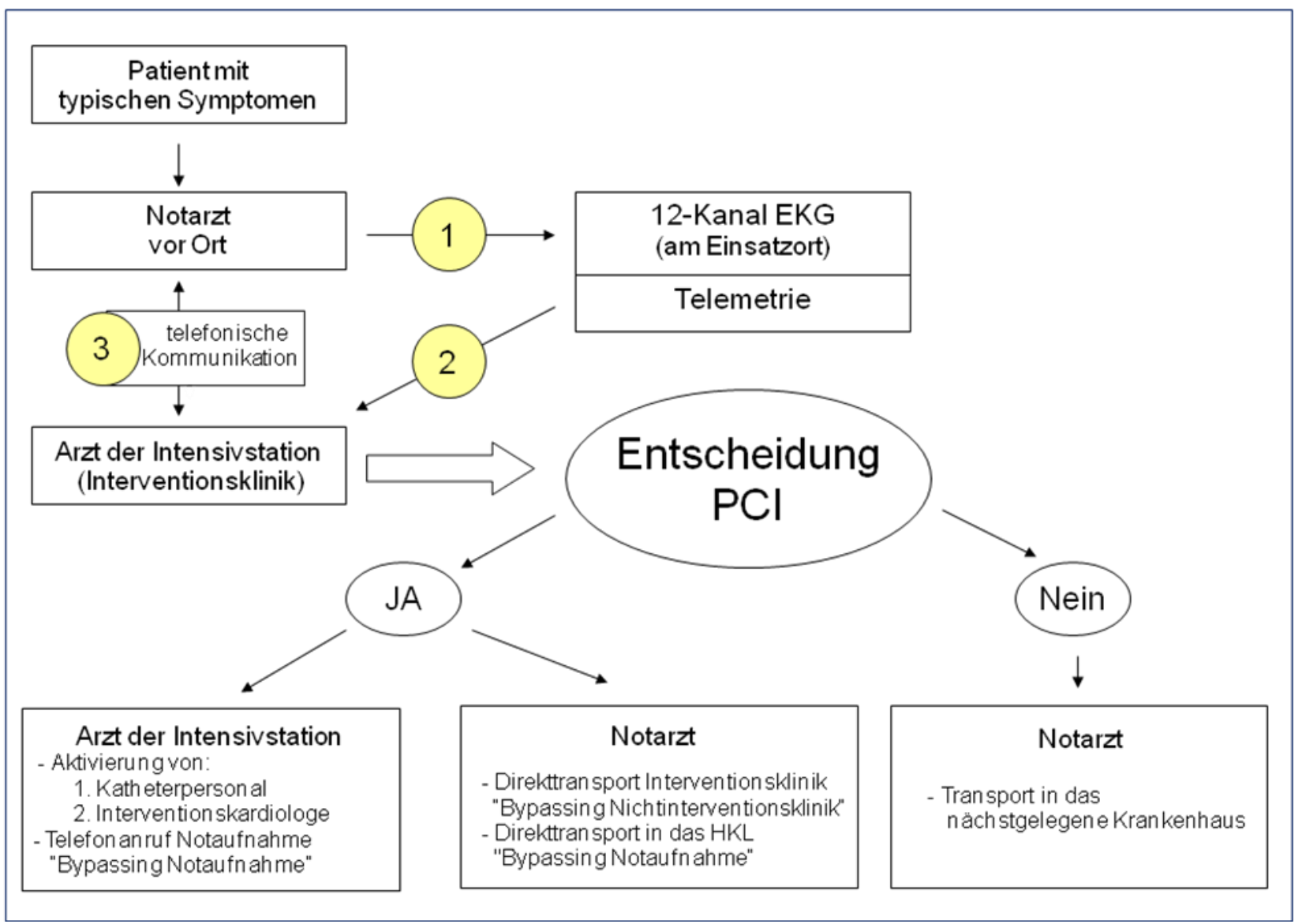

Abbildung 2: Algorithmus-Ablaufprotokoll STEMI-Behandlung im Infarktnetz Hildesheim-Leinebergland 2005

Wird anhand des übermittelten 12-Kanal-EKG jedoch keine Indikation zur sofortigen Katheterintervention gestellt, erfolgt der Transport des Patienten in die zuständige nächstliegende Nicht-Interventionsklinik. Der diensthabende Arzt der Intensivstation benachrichtigt nach Entscheidung zur Direktübernahme des Patienten unmittelbar den zuständigen Interventionskardiologen und außerhalb der regulären Dienstzeit zuerst das aus zwei Personen bestehende Assistenzpersonal des Herzkatheterlabors. Bei Eintreffen des Patienten in der Interventionsklinik wird dieser im Idealfall direkt durch den Notarzt begleitet in das HKL transportiert und an den Interventionskardiologen übergeben (Bypassing der Notaufnahme). Nur in Ausnahmefällen, in denen aufgrund von kurzen Anfahrtswegen des Patienten und fehlender Vorankündigung außerhalb der regulären Dienstzeit das HK-Personal noch nicht vor Ort ist bzw. bei paralleler Belegung beider HK-Untersuchungsräume mit Notfällen, verbleibt der Patient bis zum Beginn der Untersuchung in der Notaufnahme. 


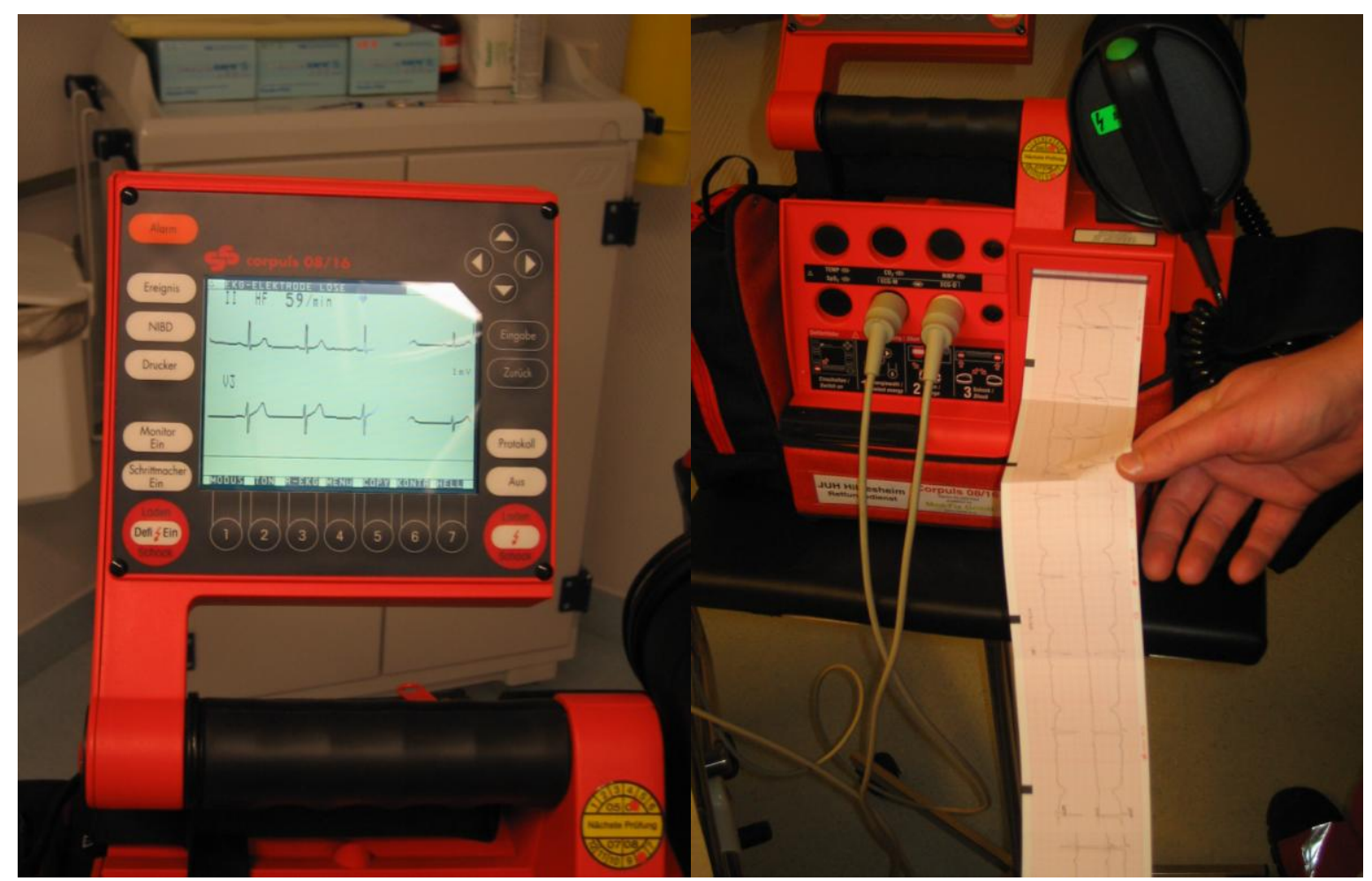

Abbildung 3: 12-Kanal-EKG „,corpuls 08/16“ 


\section{Fragestellung}

Bei der Behandlung des akuten ST-Streckenhebungsinfarkts ist die schnellstmögliche Wiedereröffnung des verschlossenen Herzkranzgefäßes wahrscheinlich von großer Bedeutung für die Prognose der Patienten. Die Leitlinien der Kardiologischen Fachgesellschaften fordern eine contact-to-balloon-Zeit von weniger als 90 min (European Society of Cardiology (ESC)) bzw. 120 min (Deutsche Gesellschaft für Kardiologie (DGK)). In den zurückliegenden Jahren wurden weltweit Anstrengungen unternommen, um die Behandlungszeiten zu verbessern. Dennoch gelingt es nach wie vor in vielen Fällen nicht, die hohen Anforderungen der Leitlinien einzuhalten.

Das regionale Herzinfarktnetz Hildesheim-Leinebergland setzt sich aus einer Interventionsklinik mit 24-h-PCI-Bereitschaft (PCI-Klinik) und 2 Nicht-Interventionskliniken mit dem dazugehörigen Rettungsdienst-System zusammen. In diesem Herzinfarktverbund besteht eine Basisstruktur mit optimalen äußeren Bedingungen (2 Herzkatheterlabore in der PCI-Klinik, sehr enge Kooperation mit den beteiligten Kliniken, Möglichkeit der Telemetrie eines Zwölf-Kanal EKG auf allen Notarzteinsatzfahrzeugen sowie ein festes Ablaufprotokoll in der Diagnose- und Therapiekette).

In der vorliegenden prospektiv durchgeführten Untersuchung sollten folgende Fragen geklärt werden:

1. Wie hoch ist der Anteil der Patienten, bei denen in einem gut organisierten Herzinfarktnetz in ländlicher Region die hohen Anforderungen der Leitlinien unter ,real-life“-Bedingungen umsetzbar sind?

2. Können die Behandlungszeiten durch standardisiertes Qualitätsmanagement mit systematischer Ergebnisrückkopplung verbessert werden? 


\section{Patienten und Methoden}

\subsection{Patientenkollektiv}

\section{Einschlusskriterien:}

In der vorliegenden prospektiven Untersuchung wurden alle diejenigen Patienten erfasst, die im Zeitraum vom 01.01.2006 bis zum 31.12.2006 im Rahmen des Herzinfarktnetzes mit den Kriterien eines ST-Streckenhebungsinfarkts und dem Ziel einer primären Koronarintervention (,intention to treat") in der Medizinischen Klinik I des St. Bernward Krankenhauses aufgenommen wurden.

Voraussetzung für den Patienteneinschluss waren eine Symptomdauer von nicht mehr als 12 Stunden und folgende elektrokardiographische Kriterien eines ST-Streckenhebungsinfarkts:

ST-Streckenhebung von $\geq 0,1 \mathrm{mV}$ in mindestens zwei zusammenhängenden Extremitätenableitungen oder

$>\geq 0,2 \mathrm{mV}$ in mindestens zwei zusammenhängenden Brustwandableitungen oder

Linksschenkelblock mit infarkttypischer Symptomatik.

\section{Ausschlusskriterien:}

Nicht in die Untersuchung eingeschlossen wurden alle diejenigen Patienten, bei denen keine Reperfusionsmaßnahme durchgeführt wurde oder durchgeführt werden konnte. 


\subsubsection{Nicht in die Auswertung eingeschlossene Patienten}

Bei Patienten mit den Charakteristika eines STEMI und ,intention to treat", bei denen keine primäre PCI erfolgte, wurden die entsprechenden Einsatzzeiten bis zum Zeitpunkt der Ankunft im Herzkatheterlabor wie bei den übrigen Patienten erfasst und analysiert. Diese STEMI-Patienten ohne PCI wurden in die Gesamtauswertung jedoch nicht einbezogen.

\subsubsection{Patientengruppen}

Bezüglich der Art der Einweisung wurden die Patienten prospektiv in drei verschiedene Gruppen eingeteilt (Tabelle 1).

\begin{tabular}{|r|l|}
\hline Primärtransport & $\begin{array}{l}\text { Transport des Patienten durch den Notarzt direkt vom Einsatzort in die } \\
\text { Interventionsklinik }\end{array}$ \\
\hline Sekundärtransport & $\begin{array}{l}\text { Verlegung aus einer Nicht-Interventionsklinik in die } \\
\text { Interventionsklinik }\end{array}$ \\
\hline $\begin{array}{r}\text { Selbstvorsteller/ } \\
\text { Selbsteinweisung }\end{array}$ & $\begin{array}{l}\text { Patienten, die sich direkt ohne Kontakt zum Notarzt /Rettungssystem } \\
\text { in der Notaufnahme der Interventionsklinik vorstellen }\end{array}$ \\
\hline
\end{tabular}

Tabelle 1: Art der Einweisung 


\subsection{Datenerfassung}

Nach stationärer Aufnahme des jeweiligen Patienten wurden die exakten zeitlichen Abläufe regelmäßig anhand eines eigens entwickelten standardisierten Zeit-Dokumentationsbogens (Abbildung 4) unmittelbar vor bzw. direkt im Anschluss an die Reperfusionsmaßnahme ermittelt. Die Einsatzzeiten des Notarztes und des Rettungsdienstes wurden den entsprechenden routinemäßig verwendeten Rettungsprotokollen entnommen. Bei unvollständig ausgefüllten Protokollen wurden die fehlenden Zeitangaben nach telefonischer Kontaktaufnahme mit der zuständigen Rettungsleitstelle, die stets simultan die Einsatzzeiten erfasst, ergänzt. Die zeitlichen Abläufe im Herzkatheterlabor wurden bereits seit Inbetriebnahme standardmäßig simultan zur durchgeführten Untersuchung exakt protokolliert und digital erfasst (Kardiologisches Informationssystem cardioBase evo). Darüber hinaus wird seit jeher bei jedem Patienten in der zentralen digitalisierten Patientendatenbank (Krankenhaus-Informationssystem Orbis) der Zeitpunkt der Vorstellung in der Zentralen Notaufnahme festgehalten. Bereits im Vorfeld waren sämtliche Beteiligte darauf hingewiesen worden, auf die korrekte Einstellung bezüglich Datum und Uhrzeit der verwendeten EKGGeräte (z.B. auch Wechsel Winter- und Sommerzeit) zu achten. Die jeweiligen Untersuchungsergebnisse, mögliche Komplikationen im Herzkatheterlabor und spezielle Bemerkungen wurden im vorliegenden Dokumentationsbogen vom untersuchenden Interventionskardiologen selbst unmittelbar eingetragen. Im Anschluss folgte noch ein direktes standardisiertes Patienteninterview, in dem zum einen der zeitliche Ablauf nochmals rekonstruiert und auf spezielle Patientencharakteristika eingegangen wurde.

Abschließend wurden die relevanten Unterlagen wie Einweisungspapiere, Notarzteinsatzprotokoll, Herzkatheterbefund und -protokoll, Erst-EKG/Funk-EKG, Anamnese- und Aufnahmeuntersuchungsbögen, Unterlagen der zuweisenden NichtInterventionskliniken, Entlassungsbriefe von jedem Patienten separat vervielfältigt und archiviert. Zur Überprüfbarkeit der Qualität der Datenerfassung wurde ergänzend ein Qualitätssicherungsbogen erstellt, mit dem bei jedem Patienten die Dokumentation dieser Quelldaten sichergestellt wird.

Die erhobenen Daten wurden in anonymisierter Form aus den Dokumentationsbögen in eine eigens vorgefertigte Datenbank (Microsoft Excel 2003) übertragen. 


\section{Dokumentationsbogen}

Primärtransport/Sekundärtransport/Selbstvorstellung

$\begin{array}{llll}\text { Datum } & : & \text { Zeitzone } & : 12 \\ \text { Telefon } & : & & 2 \\ \text { Hausarzt } & : & \text { HA-Kontakt }: \text { JA / NEIN } \\ \text { Alter } & : & & \\ \text { Gewicht } & : & \text { BMI } & :\end{array}$

Risikofaktoren:

Arterielle Hypertonie :

Diabetes mellitus :

Hyperlipoproteinämie

Nikotinabusus

BMI

Marcumar :

Ca-Antagonist :

B-Blocker :

ACE-Hemmer :

AT-1-Blocker :

Diuretikum :

Nitrat

Lipidsenker

Z.n. Apoplex

Z.n. ACVB

Vorerkrankungen:

Z.n. Myokardinfarkt

Z.n. PCI

:

Sonstige

Standort NEF

Einsatzort

:

RR syst Übergabe :

Untersucher

:

HF bei Übergabe :

Direktübergabe an Interventionskardiologen: JA / NEIN

Aktueller Symptombeginn :

Alarm NA

Notarztankunft

Funk-EKG

Abfahrt NEF

Klinikankunft

Ankunft HKL

Punktion

Balloninflation

STEMI

Angiograph. Merkmale

Rekanalisation

Kardiogener Schock
Symptom bis Klinikankunft

Alarm bis Ankunft NA

Aufenthaltsdauern NA vor Ort :

Fahrtdauer

Ankunft Klinik bis HKL

Ankunft HKL bis Punktion

Punktion bis Ballon

DOOR-TO-BALLOON

CONTACT-TO-BALLOON :

HW LW

2-G-KHK

3-G-KHK

RCX

LMCA

LMCA

CPR : JA / NEIN

IABP : JA / NEIN

Bemerkungen:

Abbildung 4: Dokumentationsbogen Infarktnetz Hildesheim-Leinebergland 2006

(Zeitzone 1=Regelarbeitszeit; Zeitzone 2: Rufbereitschaft) 


\subsection{Zeiterfassung}

Anhand der unter 3.2. beschriebenen Vorgehensweise wurden die nachfolgenden Zeitpunkte in der Rettungs- und Therapiekette erfasst und im Dokumentationsbogen festgehalten (Tabelle 2; Abbildung 4).

\begin{tabular}{|r|l|}
\hline Symptombeginn & $\begin{array}{l}\text { Beginn der Symptome bzw. Schmerzmaximum als } \\
\text { mutmaßlicher Infarktbeginn }\end{array}$ \\
\hline Alarm & Alarmierung des Notarztes durch die zuständige Rettungsleitstelle \\
\hline Ankunft & Ankunft des Notarztes am Einsatzort \\
\hline Funk-EKG & Zeitpunkt der Übermittlung des Funk-EKG \\
\hline Abfahrt & Abfahrt des Notarztes vom Einsatzort \\
\hline Übergabe & Übergabe bzw. Ankunft des Patienten in Interventionsklinik \\
\hline Ankunft HK-Labor & Ankunft des Patienten im Herzkatheterlabor, Einschleusung \\
\hline Punktion & Zeitpunkt Punktionsbeginn A. femoralis rechts bzw. links \\
\hline Dilatation & Erste Balloninflation im Bereich der Verschluss-Stelle \\
\hline
\end{tabular}

Tabelle 2: Erfasste Zeitpunkte im Dokumentationsbogen

Aus diesen ermittelten Zeitpunkten konnten die in Tabelle 3 definierten Zeitintervalle rechnerisch ermittelt und anhand ihres chronologischen Ablaufs gegliedert werden (Tabelle 3).

\begin{tabular}{|r|l|}
\hline Alarm bis Notarztankunft & $\begin{array}{l}\text { Eingang des Notrufs bis Ankunft des Notarztes am } \\
\text { Einsatzort }\end{array}$ \\
\hline Aufenthaltsdauer & Ankunft des Notarztes am Einsatzort bis Abfahrt \\
\hline Transportdauer & $\begin{array}{l}\text { Abfahrt des Notarztes vom Einsatzort bis Übergabe des } \\
\text { Patienten in der Interventionsklinik }\end{array}$ \\
\hline $\begin{array}{r}\text { Ankunft Klinik bis HKL } \\
\text { Ankunft HKL bis Punktion } \\
\text { (=Vorbereitung HKL) }\end{array}$ & $\begin{array}{l}\text { Ankunft des Patienten in Interventionsklinik bis Ankunft } \\
\text { im Herzkatheterlabor }\end{array}$ \\
\hline $\begin{array}{r}\text { Punktion bis Ballon } \\
\text { (=Therapie im HKL) }\end{array}$ & Punktion der A. femoralis bis erste Balloninflation im \\
Infarktgefäß
\end{tabular}

Tabelle 3: Zeitintervalle der Rettungs- und Therapiekette 
Aus dem Zusammenschluss der entsprechenden Zeitintervalle konnten die Prähospitalzeit sowie die Revaskularisationszeiten (door-to-balloon-Zeit und contact-to-balloon-Zeit) ermittelt werden (Tabelle 4).

\begin{tabular}{|r|l|}
\hline Prähospitalzeit & $\begin{array}{l}\text { Symptombeginn (mutmaßlicher Infarktbeginn) bis } \\
\text { Ankunft des Patienten in der Interventionsklinik }\end{array}$ \\
\hline door-to-balloon & $\begin{array}{l}\text { Ankunft des Patienten in Interventionsklinik bis erste } \\
\text { Balloninflation im Infarktgefäß }\end{array}$ \\
\hline contact-to-balloon & $\begin{array}{l}\text { Erstkontakt des Patienten mit dem Arzt/Notarzt bis erste } \\
\text { Balloninflation im Infarktgefäß }\end{array}$ \\
\hline
\end{tabular}

Tabelle 4: Präshospitalzeit und Revaskularisationszeiten (door-to-balloon und contact-to-balloon)

Zusätzlich wurde bei Patienten mit Sekundärtransport differenziert, ob der Patient zuvor mit Notarzt, Rettungswagen oder als Selbsteinweisung/Selbstvorsteller in die NichtInterventionsklinik aufgenommen worden war. Die sich daraus ergebenden weiteren Einsatzzeiten wurden in Analogie zu den bereits o.g. ebenfalls erfasst:

- Symptombeginn bis Ankunft Nicht-Interventionsklinik

- Alarm bis Notarztankunft (Ersttransport)

- Aufenthaltsdauer am Einsatzort (Ersttransport)

- Transportdauer (Ersttransport).

Darüber hinaus wurde die Zeitdauer zwischen Ankunft in der Nicht-Interventionsklinik und Ankunft des Patienten in der Interventionsklinik (door-to-door) gesondert ermittelt.

Im Fall einer Selbstvorstellung in Interventions- oder Nichtinterventionsklinik wurde entgegen den bisherigen Angaben nicht der Arztkontakt als Erstkontakt zur Berechnung der contact-to-balloon-Zeit herangezogen, sondern der Zeitpunkt der Ankunft der Patienten in der Notaufnahme der jeweiligen Klinik. So findet auch die Wartezeit des sich mit typischer Beschwerdesymptomatik vorstellenden Patienten bis zum Arztkontakt und Diagnosestellung Berücksichtigung. 
Ferner wurden diejenigen Patienten, die nur mittels Rettungswagen ohne Arztbegleitung in die jeweilige Klinik verbracht worden waren, ebenfalls der Gruppe der Selbstvorsteller zugeordnet, da bei diesen Patienten keine telefonische Vorankündigung bzw. Telemetrie eines EKG stattgefunden hatte und letztendlich weder die (Verdachts-)Diagnose eines STEMI gestellt noch in Abwesenheit eines Notarztes die entsprechenden therapeutischen Schritte eingeleitet werden konnten.

Zur Vergleichbarkeit der erhobenen Daten wurden für die Ermittlung der contact-to-balloonZeit grundsätzlich die Ankunft des Notarztes beim Patienten respektive die Ankunft des Patienten in der jeweiligen Notaufnahme zugrunde gelegt. Ein ggfs. vorher stattgefundener Hausarzt-Kontakt wurde nicht als Erstkontakt gewertet. Die Art des Kontakts zum Hausarzt respektive zum kassenärztlichen Notdiensts (KÄND) wurde jedoch im zeitlichen und qualitativen Kontext miterfasst:

- Telefonische Kontaktaufnahme und Beratung

- Hausbesuch beim Patienten mit Beratung bzw. Alarmierung des Notarztes

- Vorstellung in der Praxis des Hausarztes mit EKG-Erstellung und Diagnosesicherung - Symptombeginn bis Kontakt mit Hausarzt bzw. KÄND.

Sofern reproduzierbar wurde die Zeit zwischen Hausarztkontakt und Ankunft des Notarztes bzw. Ankunft in der aufnehmenden Klinik zusätzlich ermittelt. 


\subsection{Basischarakteristika}

Im Folgenden sind noch weitere Basischarakteristika aufgelistet, die für jeden Patienten gesondert ermittelt wurden (Tabelle 5).

\begin{tabular}{|r|l|}
\hline Alter & \\
\hline Geschlecht & \\
\hline Zeitpunkt der Intervention & 1 (werktags 7:30-16:00) \\
2 (werktags 16:00-7:30 und an den Wochenenden)
\end{tabular}

Tabelle 5: Definition Basischarakteristika 
In der Gruppe der Primärtransporte wurde zusätzlich der Standort des jeweiligen Notarzteinsatzfahrzeugs (St. Bernward Krankenhaus, Klinikum Hildesheim, Krankenhaus Alfeld, Krankenhaus Gronau, andere Standorte) registriert. Zusätzlich wurde prospektiv erfasst, ob ein Funk-EKG übermittelt wurde und ob eine Direktübergabe durch den Notarzt an den Interventionskardiologen (Umgehung/Bypassing der Notaufnahme) stattgefunden hat.

Bezüglich des Einsatzorts wurde die geographische Lage innerhalb des Landkreises Hildesheim (Stadt vs. Land) mit entsprechender Entfernung zur Nicht-Interventionsklinik bzw. Interventionsklinik bestimmt und graphisch dargestellt. Darüber hinaus wurde zwischen den einzelnen Untersuchern unterschieden. Gesondert wurden individuell bei jedem Patienten unmittelbar nach erfolgter Intervention durch den Untersucher selbst mögliche Gründe für eine Zeitverzögerung im Behandlungsablauf, wenn das vorgegebene Zeitfenster der „contactto-balloon“ Zeit von 90 min überschritten worden war, im Einzelfall prospektiv erfasst.

Abschließend wurde neben der Krankenhausmortalität die Hospitalisierungszeit ermittelt. Im Rahmen des Herzinfarktnetzes mit Kooperation der beteiligten Kliniken waren wir bemüht, die Patienten, die als Sekundärtransport aus eine der Nicht-Interventionskliniken in unsere Klinik transportiert worden waren, möglichst zeitnah wieder heimatnah zurückzuverlegen. Genauso verhielt es sich mit den Primärtransporten aus dem Einzugsgebiet der NichtInterventionskliniken, die nach Möglichkeit nach erfolgter Intervention und entsprechender Überwachungszeit in das verantwortliche Regionskrankenhaus zurückverlegt wurden. Die Anzahl der rückverlegten Patienten wurde quartalsweise erfasst und auf den FeedbackVeranstaltungen präsentiert. 


\subsection{Projektablauf}

Im Vorfeld wurde das geplante Projekt im Rahmen einer gemeinsamen Einführungsveranstaltung (am 15.11.05) den ärztlichen Leitern der Rettungsdienste und den Chefärzten der beteiligten Kliniken in Alfeld und Gronau vorgestellt. Dabei wurden die generellen Anforderungen an das Herzinfarktnetz nochmals fokussiert:

Funkübermittlung eines 12-Kanal-EKG (bei jedem Pat. gefordert, möglichst als Erstmaßnahme auch bei kurzem Anfahrtsweg)

$>$ „Bypassing“ der Nicht-Interventionsklinik (Vermeidung von Sekundärtransporten innerhalb des Herzinfarktnetzes)

$>$ „Bypassing“ der Notaufnahme der Interventionsklinik (möglichst direktes Anfahren des HKL ohne Aufenthalt auf der Notaufnahme und Direktübergabe des Patienten durch den Notarzt an den Interventionskardiologen im HKL).

Bei allen Patienten, die die o.g. Einschlusskriterien erfüllten, wurden die zeitlichen Abläufe in der Akutphase des STEMI von Symptombeginn bis Wiedereröffnung des verschlossenen Herzkranzgefässes sowie die weiteren oben genannten Patientencharakteristika systematisch und prospektiv erfasst. Im Anschluss erfolgte die anonymisierte Eingabe in eine ExcelDatenbank sowie die grafische Auswertung. Die Ergebnisse wurden mit Beginn am 01.01.2006 quartalsweise erfasst, die Zwischenauswertungen wurden zeitnah nach dem jeweiligen Quartalsabschluss allen an der Rettungs- und Therapiekette beteiligten Gruppen im Rahmen einer gemeinsamen Veranstaltung mittels eines interaktiven Vortrags systematisch präsentiert. Das erste Quartal wurde als Referenzquartal für die nachfolgenden Quartale (=Interventionsquartale 1-3) zugrunde gelegt. Im Rahmen der Datenpräsentation wurde jeweils die Pathophysiologie der Infarktentstehung und -ausdehnung, Therapieoptionen und empfehlungen anhand der aktuellen Leitlinien sowie Auswirkungen von Zeitverlust und Zeitgewinn auf die Infarktgröße und Prognose der betroffenen Patienten thematisiert. Im nächsten Schritt wurden gemeinsam Problemfelder mit mutmaßlichem Verbesserungspotential fokussiert. Gleichzeitig dienten die Veranstaltungen der Integration aller an der Rettungs- und Therapiekette beteiligten Gruppen mit dem Versuch dadurch einen hohen Identifikationsgrad aller Teilnehmer mit dem Projekt „Herzinfarktnetz“ zu erzielen (,Wir-Gefühl, „Erlebnis einer großen Familie“). 
Diese Rückkopplungs-Veranstaltungen, die von der Akademie für ärztliche Fortbildung mit jeweils 2 Fortbildungspunkten anerkannt waren, wurden durchschnittlich von 30-40 Teilnehmern bestehend aus Notärzten, Rettungsassistenten der Region, Ärzten aller beteiligten Kliniken, Teams der Notaufnahme, Intensivstation und Herzkatheterlabor besucht. Bezogen auf die Ergebnispräsentation wurde einheitlich vorgegangen:

Ausführliche und interaktive Präsentation der Quartalsresultate sowohl der Gesamtgruppe als auch separat der Gruppe der Primärtransporte, Sekundärtransport und der sich ohne Vorankündigung vorstellenden Patienten in Form der relevanten Zeitintervalle (Aufenthaltsdauer am Einsatzort, Transportdauer, Aufenthalt in der Notaufnahme, Vorbereitung im HKL, Interventionsdauer im HKL mit den daraus resultierenden Zeiten für door-to-balloon und contact-to-balloon)

Kasuistische Präsentation von prospektiv erfassten Ursachen für die bei einzelnen Patienten eingetretenen Zeitverzögerungen innerhalb der Rettungs- und Therapiekette (problembezogene Hinterfragung stark abweichender Ergebnisse)

> Systematische Fokussierung auf Bereiche mit mutmaßlich noch vorhandenem Optimierungspotential, Identifikation von noch nicht ausgeschöpften Ressourcen und möglichen Schwachstellen

Wiederholung der generellen Anforderungen innerhalb des Herzinfarktnetzes (Funkübermittlung eines 12-Kanal-EKG; Bypassing der Nicht-Interventionsklinik, Bypassing der Notaufnahme im Interventionskrankenhaus). 


\subsection{Chronologische Projektdurchführung}

16.11.2005: $\quad$ Vorstellung d. Projekts i. R. des Herzinfarktnetzes

(Leineberglandkliniken Alfeld)

16.12.2005: $\quad$ Genehmigung und Befürwortung des Projekts durch die

Ethikkommission der Universität Göttingen

01.01.-31.03.2006: Datenerfassung I. Quartal (=Referenzquartal)

11.04.2006: $\quad$ Präsentation der Ergebnisse des Referenzquartals

(Leineberglandkliniken Alfeld)

01.04.-30.06.2006: Datenerfassung II. Quartal (=Interventionsquartal 1)

27.06.2006: Interdisziplinäre Rettungsdienstfortbildung“

Wdh.: Präsentation der Ergebnisse des Referenzquartals

17.07.2006: $\quad$ Präsentation der Ergebnisse des Interventionsquartals 1

(Leineberglandkliniken Gronau)

01.07.-30.09.2006: Datenerfassung III. Quartal (=Interventionsquartal 2)

09.10.2006: $\quad$ Präsentation der Ergebnisse des Interventionsquartals 2

(Leineberglandkliniken Alfeld)

01.07.-31.12.2006: Datenerfassung IV. Quartal (=Interventionsquartal 3)

16.01.2007: $\quad$ Präsentation der Ergebnisse des Interventionsquartals 3

(St. Bernward Krankenhaus Hildesheim)

\subsection{Qualitätsmanagement-Interventionen}

Nach exakter Prozessanalyse fanden nachfolgende Interventionen im Kontext der kontinuierlichen Qualitätsverbesserung Anwendung:

Systematisches Feedback durch Präsentation der Quartalsergebnisse

Definition und Etablierung von Maßnahmen zur weiteren Qualitätsverbesserung

Versuch der Integration aller beteiligten Systeme: Hohe Identifikation mit dem Projekt. 


\subsection{Kern-Qualitätsindikatoren}

Vor Beginn der Untersuchung wurden zur Messbarkeit der Qualitätsverbesserung die nachfolgenden Kern-Qualitätsindikatoren definiert und prospektiv zur Messung der Ablaufqualität in der Rettungs- und Therapiekette sowie zur Erfolgskontrolle eingesetzt (Quantifizierung des QM-Interventionserfolgs):

Aufenthaltsdauer des Notfall-Teams am Einsatzort (= Ankunft NEF am Einsatzort bis Abfahrt)

Aufenthaltsdauer des Patienten in der Notaufnahme der Interventionsklinik (= Ankunft Klinik bis Ankunft HKL)

Anteil der direkt vom Notarzt an den Interventionskardiologen übergebenen Patienten

Dauer der Vorbereitung im HKL (Einschleusung bis Punktionsbeginn)

Dauer der Therapie im HKL (Punktion bis 1. Balloninflation)

Anteil der Patienten mit übermitteltem Funk-EKG

Anteil der Patienten mit Sekundärtransport

Anteil der Patienten mit einer contact-to-balloon Zeit $<90$ bzw. $<120$ min.

\subsection{Verstorbene Patienten}

Die während der Hospitalisierung verstorbenen STEMI-Patienten wurden mit Angabe von Basischarakteristika (Alter, Geschlecht, Art der Einweisung), den ermittelten Revaskularisationszeiten (door-to-balloon und contact-to-balloon) sowie Hospitalisierungszeit und Todesursache ergänzend gesondert analysiert. 


\subsection{Statistische Auswertung}

Die Analyse und statistische Auswertung der erhobenen Daten wurden in Zusammenarbeit mit einem unabhängigen statistischen Institut durchgeführt (Abteilung für Medizinische Statistik der Universität Göttingen, Prof. Dr. Hilgers).

Die Verteilungen der Zeitintervalle wurden grafisch als Kaplan-Meier-Schätzer-Kurven präsentiert. Zum Vergleich der einzelnen Quartale wurde der Gehan-Test verwendet, welcher für unzensierte Daten äquivalent zum Kruskal-Wallis-Test bzw. Wilcoxon-Mann-WhitneyTest ist. Als Maß der zentralen Tendenz wurde jeweils der Median bestimmt. Kategoriale Daten wurden als Häufigkeiten und Prozente angegeben, für den Gruppenvergleich wurde der Pearson $\mathrm{Chi}^{2}$-Test durchgeführt. Als statistische Software wurde STATISTICA 7.1 (StatSoft, Inc. Tulsa OK 2005) verwendet. 


\section{Ergebnisse}

\subsection{Patienten}

Innerhalb des zwölfmonatigen Untersuchungszeitraums (01.01.2006-31.12.2006) wurden insgesamt 120 Patienten mit den Charakteristika eines ST-Streckenhebungsinfarkts und dem Ziel einer primären PCI in das Herzkatheterlabor des St. Bernward Krankenhauses transportiert (,,intention-to-treat"‘).

\subsubsection{Nicht in die Auswertung eingeschlossene Patienten}

Im gesamten Untersuchungszeitraum wurde bei 6 Patienten mit den klinischen Kriterien eines STEMI und ,intention to treat“ im Verlauf keine Akut-PCI durchgeführt. Die Daten dieser Patienten wurden nicht in die endgültige Auswertung einbezogen. In einem Fall wurde bei gleichzeitig bestehendem ausgedehnten linkshirnigen Apoplex auf ein interventionelles Vorgehen verzichtet. Bei einem weiteren Patienten wurde die Koronarangiographie unter laufenden Wiederbelebungsmaßnahmen durchgeführt, bei schließlich frustranen Reanimationsbemühungen und komplexer Ausgangssituation mit Zustand nach operativer Revaskularisation gelang keine primäre Koronarintervention. In vier weiteren Fällen konnte kein Zielgefäß (culprit lesion) ausgemacht werden. Bei zwei dieser Patienten führte man die ST-Streckenhebungen auf eine Myokarditis zurück, ein weiterer Patient bot während der Untersuchung einen TIMI III - Fluss ohne verbliebene Reststenose im vermuteten Zielgefäß. Beim vierten dieser Patienten ohne Zielgefäß für eine Intervention lag ein ausgeprägter koronarer Vasospasmus vor, der sich auf intrakoronare Nitro-Applikation vollständig löste. 
Die Daten- und Zeiterfassung dieser letztlich nicht in die endgültige Auswertung einbezogenen Patienten wurde bis zur Ankunft im Herzkatheterlabor bzw. bis zur Gefäßpunktion in identischer Weise wie bei allen übrigen Patienten vorgenommen (Tabelle $6)$.

\begin{tabular}{|c|c|c|c|c|c|c|}
\hline & \multicolumn{3}{|c|}{ I. Quartal } & \multirow{2}{*}{\begin{tabular}{|c|}
$\begin{array}{c}\text { II. } \\
\text { Quartal }\end{array}$ \\
Patient 4 \\
\end{tabular}} & \multicolumn{2}{|l|}{ IV. Quartal } \\
\hline No. & Patient 1 & Patient 2 & Patient 3 & & Patient 5 & Patient 6 \\
\hline Alter (Jahre) & 75 & 49 & 75 & 91 & 77 & 71 \\
\hline $\begin{array}{l}\text { Geschlecht } \\
\text { (M/F) }\end{array}$ & $\mathrm{F}$ & M & $F$ & M & M & M \\
\hline $\begin{array}{c}\text { Art der } \\
\text { Einweisung }\end{array}$ & ST & SV & ST & PT & PT & PT \\
\hline $\begin{array}{l}\text { Ankunft Klinik } \\
\text { bis HKL (min) }\end{array}$ & 6 & 34 & 5 & 2 & 1 & 3 \\
\hline $\begin{array}{l}\text { Erst-Kontakt bis } \\
\text { Ankunft HKL } \\
\text { (min) }\end{array}$ & 90 & 34 & 98 & 75 & 49 & 31 \\
\hline $\begin{array}{l}\text { Begründung für } \\
\text { nicht-erfolgte } \\
\text { Rekanalisation }\end{array}$ & $\begin{array}{c}\text { Kein Zielgefäß } \\
\text { bei V.a. } \\
\text { Myokarditis }\end{array}$ & Vasospastik & $\begin{array}{l}\text { Konservativ bei } \\
\text { ausgeprägter } \\
\text { linkshirniger } \\
\text { Ischämie }\end{array}$ & $\begin{array}{l}\text { TIMI III ohne } \\
\text { relevante } \\
\text { Reststenose } \\
\text { (Thromben) }\end{array}$ & $\begin{array}{c}\text { Untersuchung } \\
\text { unter } \\
\text { Reanimation, } \\
\text { Z.n. ACVB, } \\
\text { keine Intervention } \\
\text { möglich }\end{array}$ & $\begin{array}{c}\text { Kein } \\
\text { Zielgefäß bei } \\
\text { V.a. } \\
\text { Myokarditis }\end{array}$ \\
\hline
\end{tabular}

Tabelle 6: Nicht in die Auswertung eingeschlossene Patienten

(*PT=Primärtransport, $S T=$ Sekundärtransport, $S V=$ Selbstvorsteller)

\subsubsection{Eingeschlossene Patienten}

Somit wurden insgesamt 114 Patienten in die endgültige Analyse eingeschlossen. In Abbildung 5 ist die Anzahl der Patienten bezogen auf alle 4 Quartale, sowie die Zuordnung bezüglich der Art der Einweisung (Primärtransport, Sekundärtransport und Selbstvorstellung in der PCI-Klinik) aufgeführt. Das Basisquartal setzte sich aus 33 Patienten zusammen, im nachfolgenden II, III. und IV. Quartal betrugen die Patientenzahlen 28, 25 und 28 Patienten. Den jeweils größten Anteil stellte die Gruppe der Primärtransporte mit einem prozentualen Anteil von 67 bis maximal 76\% im Quartalsvergleich dar (n=22, 22, 19, 21 in Quartal I bis IV). Die Anzahl der aus einer der Nicht-Interventionskliniken verlegten Patienten war von initial 21\% im I. Quartal auf 14\% im IV. Quartal tendenziell rückläufig (Abbildung 5). 
Im III. und IV. Quartal befand sich in der Gruppe der Sekundärtransporte kein Patient mehr, der zuvor notarztbegleitet in eine der Nicht-Interventionskliniken verbracht worden war. Die Patienten hatten sich größtenteils direkt in der dortigen Notaufnahme vorgestellt oder waren im RTW ohne Notarztbegleitung in der jeweiligen Klinik eingetroffen. Die Gruppe der Selbstvorsteller in der PCI-Klinik betrug 9,6\% am Gesamtkollektiv.

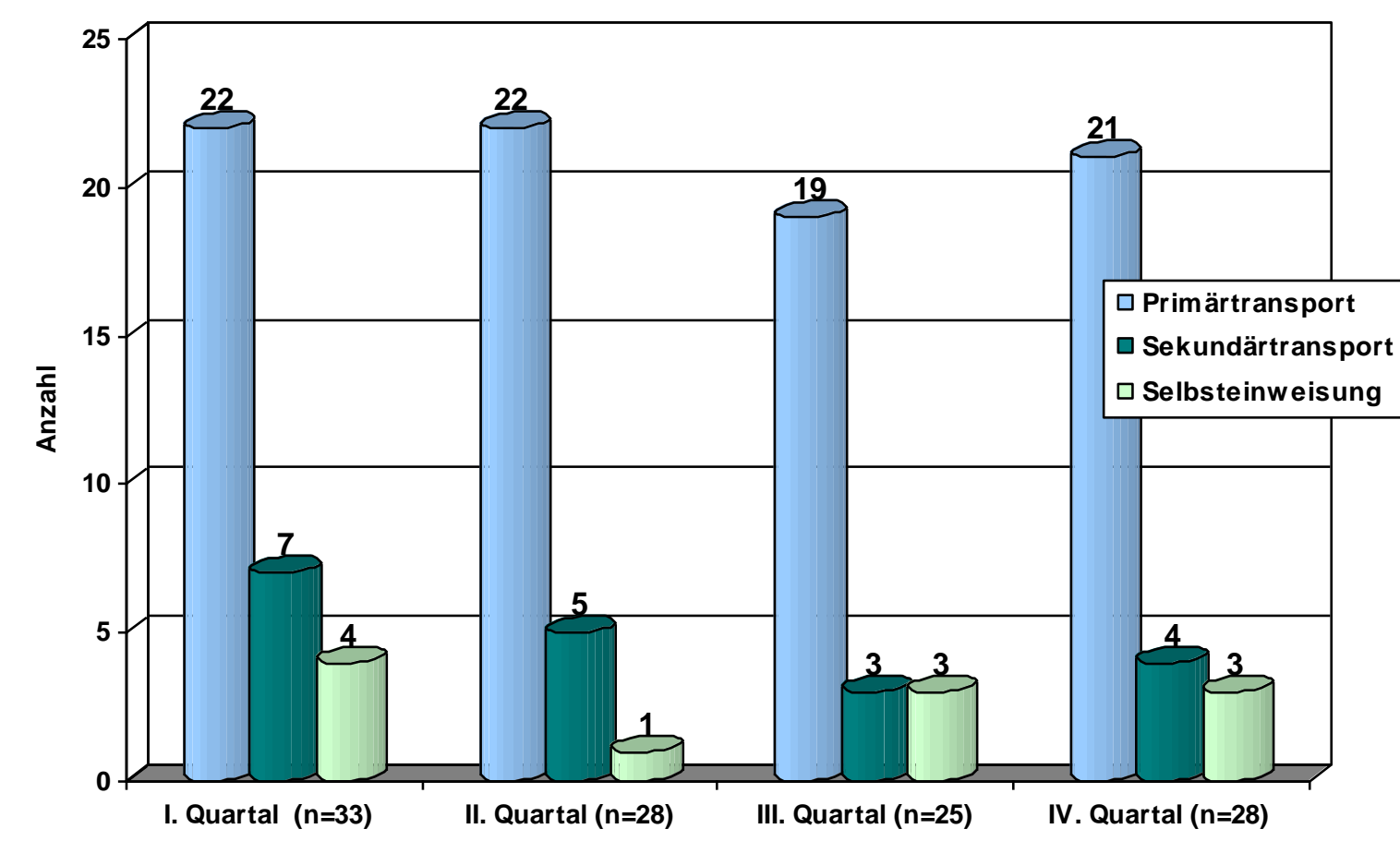

Abbildung 5: Anzahl der eingeschlossenen Patienten ( $n=114)$ im Quartalsvergleich, unterteilt nach Art der Einweisung

\subsubsection{Basischarakteristika}

Die insgesamt 114 eingeschlossenen Patienten waren durchschnittlich 66 Jahre alt und in 63\% der Fälle männlich. Der überwiegende Teil der Gruppe der Primärtransporte (71\%) stammte nicht aus dem Stadtgebiet, sondern wurde aus dem Landkreis und anliegenden Regionen in die Interventionsklinik transportiert. Über den gesamten Beobachtungszeitraum fielen etwa $50 \%$ der Infarktbehandlungen in die Bereitschaftsdienstzeit.

Die in Tabelle 7 aufgeführten Basischarakteristika waren zwischen den 4 Quartalen nicht signifikant verschieden. 


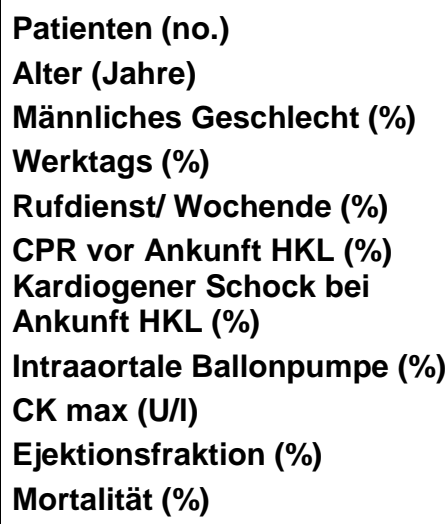

\begin{tabular}{|c|c|c|c|c|c|}
\hline & Quartal I & Quartal II & Quartal III & Quartal IV & p-Wert \\
\hline Patienten (no.) & 33 & 28 & 25 & 28 & \\
\hline Alter (Jahre) & $63 \pm 13.5$ & $68 \pm 12.9$ & $66 \pm 10.6$ & $65 \pm 15.8$ & 0.42 \\
\hline Männliches Geschlecht (\%) & $18(54.5)$ & $16(57.1)$ & $16(64.0)$ & $22(78.6)$ & 0.23 \\
\hline Werktags (\%) & $16(48.5)$ & $16(57.1)$ & $13(52.0)$ & $14(50.0)$ & 0.92 \\
\hline Rufdienst/ Wochende (\%) & $17(51.5)$ & $12(42.9)$ & $12(48.0)$ & $14(50.0)$ & 0.92 \\
\hline $\begin{array}{l}\text { CPR vor Ankunft HKL (\%) } \\
\text { Kardiogener Schock bei }\end{array}$ & $4(12.1)$ & $1(3.6)$ & $2(8.0)$ & - & 0.23 \\
\hline Ankunft HKL (\%) & $5(15.2)$ & $3(10.7)$ & $4(16.0)$ & $4(14.3)$ & 0.95 \\
\hline Intraaortale Ballonpumpe (\%) & $3(9.1)$ & - & $1(4.0)$ & $2(7.1)$ & 0.42 \\
\hline CK $\max (\mathrm{U} / \mathrm{I})$ & $1794 \pm 1386$ & $1954 \pm 1410$ & $1466 \pm 1160$ & $1949 \pm 1769$ & 0.68 \\
\hline Ejektionsfraktion (\%) & $51 \pm 12$ & $56 \pm 9$ & $54 \pm 13$ & $52 \pm 12$ & 0.46 \\
\hline \multicolumn{6}{|l|}{ Mortalität (\%) } \\
\hline 30 Tage & $3(9)$ & $1(4)$ & $3(12)$ & $2(7)$ & 0.71 \\
\hline 6 Monate & $4(12)$ & $1(4)$ & $3(12)$ & $4(14)$ & 0.57 \\
\hline Body-mass-index (BMI) & $28 \pm 4.9$ & $27 \pm 4.7$ & $26 \pm 3.1$ & $27 \pm 3.4$ & 0.30 \\
\hline Arterielle Hypertonie (\%) & $20(60.6)$ & 19 (67.9) & $13(52.0)$ & $23(82.1)$ & 0.12 \\
\hline Diabetes (\%) & $8(24.2)$ & $3(10.7)$ & $4(16.0)$ & $5(17.9)$ & 0.58 \\
\hline Rauchen (\%) & $15(45.5)$ & $8(28.6)$ & $7(28.0)$ & $5(17.9)$ & 0.13 \\
\hline Z.n. Myokardinfarkt & $6(18.2)$ & $1(3.6)$ & $2(8.0)$ & $8(28.6)$ & 0.04 \\
\hline Z.n. PTCA (\%) & $2(6.1)$ & $1(3.6)$ & $2(8.0)$ & $4(14.3)$ & 0.49 \\
\hline Z.n. Apoplex (\%) & $2(6.1)$ & $1(3.6)$ & $1(4.0)$ & $2(7.1)$ & 0.92 \\
\hline Angiographische Merkmale (\%) & & & & & 0.34 \\
\hline Eingefäß-KHK & $8(24.2)$ & $11(39.3)$ & $8(32.0)$ & $9(32.1)$ & \\
\hline Zweigefäß-KHK & $14(42.4)$ & $4(14.3)$ & $10(40.0)$ & $9(32.1)$ & \\
\hline Dreigefäß-KHK & $11(33.3)$ & $13(46.4)$ & $7(28.0)$ & $10(35.7)$ & \\
\hline Hauptstammbeteiligung & $3(9.1)$ & $1(3.6)$ & $1(4.0)$ & - & 0.38 \\
\hline Vorderwandmyokardinfarkt (\%) & $16(48.5)$ & $9(32.1)$ & $7(28.0)$ & $13(46.4)$ & 0.30 \\
\hline \multicolumn{6}{|l|}{ Infarktgefäß (\%) } \\
\hline LAD & $15(45.5)$ & $9(32.1)$ & $7(28.0)$ & $13(46.4)$ & 0.38 \\
\hline RCA & $12(36.4)$ & $14(50.0)$ & $16(64.0)$ & $11(39.3)$ & 0.16 \\
\hline RCX & $3(9.1)$ & $5(17.9)$ & $2(8.0)$ & $3(10.7)$ & 0.65 \\
\hline LMCA & $2(6.1)$ & - & - & - & 0.17 \\
\hline ACVB & $1(3.0)$ & - & - & $1(3.6)$ & 0.62 \\
\hline Herzfrequenz (Schläge/min) & $74 \pm 17.2$ & $72 \pm 15.9$ & $72 \pm 17.4$ & $80 \pm 15.7$ & 0.26 \\
\hline Systolischer Blutdruck (mmHg) & $130 \pm 29.2$ & $141 \pm 25.8$ & $123 \pm 22.2$ & $128 \pm 23.9$ & 0.12 \\
\hline \multicolumn{6}{|l|}{ Vormedikation (\%) } \\
\hline ASS & $5(15.2)$ & $5(17.9)$ & $5(20.0)$ & $6(21.4)$ & 0.93 \\
\hline B-Blocker & $10(30.3)$ & $12(42.9)$ & $8(32.0)$ & $12(42.9)$ & 0.63 \\
\hline ACE-Hemmer & $9(27.3)$ & $6(21.4)$ & $7(28.0)$ & $8(28.6)$ & 0.93 \\
\hline AT-1-Blocker & - & $2(7.1)$ & $3(12.0)$ & $2(7.1)$ & 0.29 \\
\hline Calciumantagonisten & $2(6.1)$ & $7(25.0)$ & $2(8.0)$ & $3(10.7)$ & 0.12 \\
\hline Nitrate & $1(3.0)$ & $3(10.7)$ & $1(4.0)$ & $1(3.6)$ & 0.52 \\
\hline Diuretika & 7 (21.2) & $6(21.4)$ & $5(20.9)$ & $8(28.6)$ & 0.87 \\
\hline Lipidsenker & $2(6.1)$ & $4(14.3)$ & $6(24.0)$ & $7(25.0)$ & 0.16 \\
\hline Coumarine & $3(9.1)$ & $1(3.6)$ & $1(4.0)$ & $1(3.6)$ & 0.71 \\
\hline Primärtransport (\%) & $22(66.7)$ & $22(78.6)$ & $19(76.0)$ & $21(75.0)$ & 0.85 \\
\hline Stadt (\%) & $8(36.4)$ & $6(27.3)$ & $5(26.3)$ & $5(23.8)$ & 0.81 \\
\hline Land (\%) & $14(63.6)$ & $16(72.7)$ & $14(73.7)$ & $16(76.2)$ & \\
\hline Sekundärtransport (\%) & 7 (21.2) & $5(17.9)$ & $3(12.0)$ & $4(14.3)$ & 0.85 \\
\hline Selbsteinweisung (\%) & $4(12.1)$ & $1(3.6)$ & $3(12.0)$ & $3(10.7)$ & 0.85 \\
\hline
\end{tabular}

Tabelle 7: Auswertung Basischarakteristika 


\subsubsection{Herkunft der Patienten}

In den Abbildungen 6-9 ist die Herkunft der Patienten innerhalb des Landkreises quartalsweise dargestellt, wobei zwischen Primär-/-Sekundärtransport und Selbsteinweisung farblich unterschieden wird. Im Fall der Sekundärtransporte ist der Weg vom Einsatzort bis in die jeweilige Nicht-Interventionsklinik mit einem Pfeil räumlich dargestellt. Diese Landkarten waren Bestandteil und Diskussionsgrundlage der quartalsweise vorgenommenen Ergebnispräsentationen.

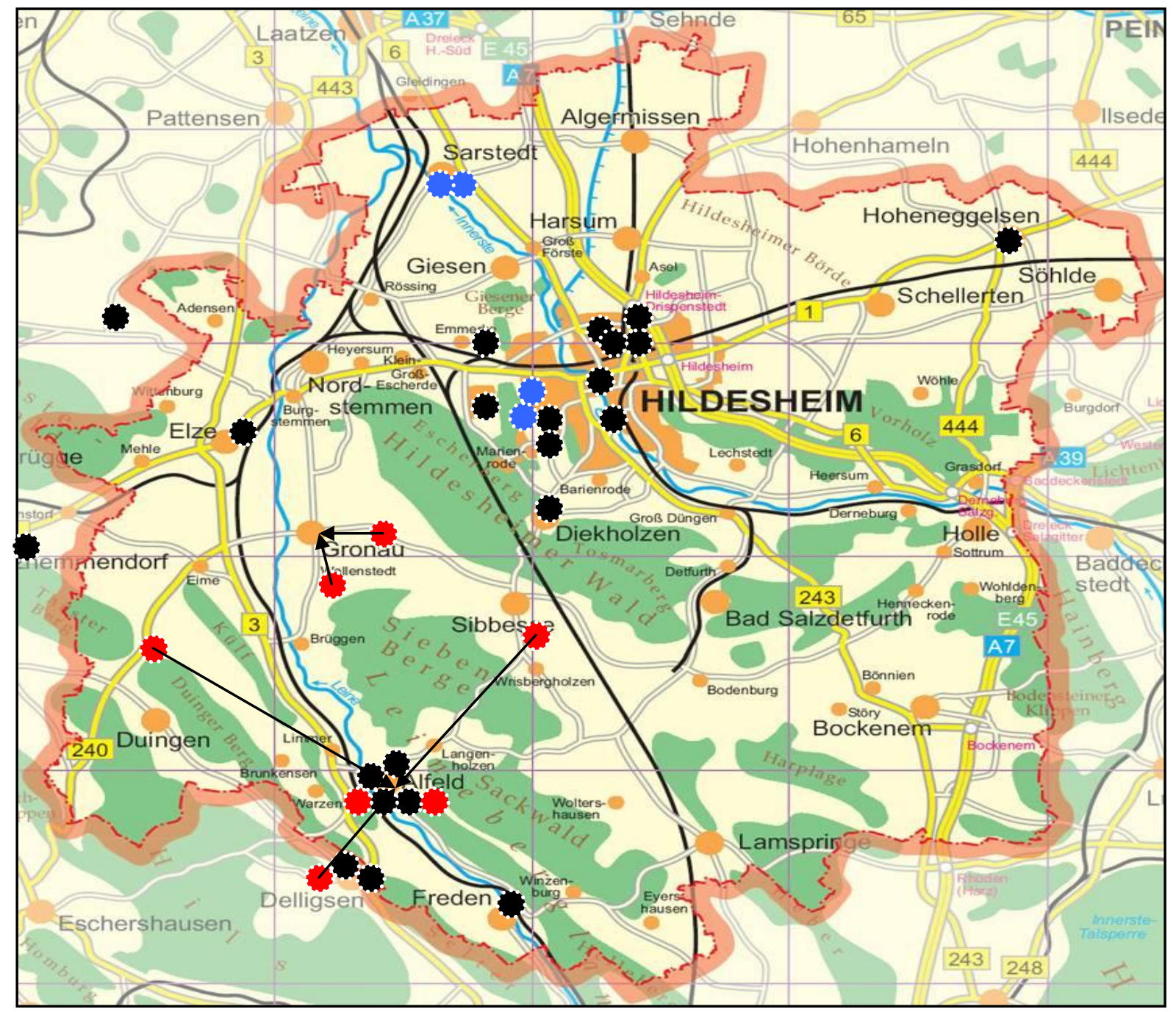

Abbildung 6: Herkunft Patienten I. Quartal (schwarz=Primärtransport, rot=Sekundärtransport, blau=Selbsteinweisung) 
Im I. Quartal wurde etwa 1/3 der Patienten aus der Gruppe der Primärtransporte aus dem Stadtgebiet in die Interventionsklinik transportiert, der weiteste Anfahrtsweg (aus dem im süd-westlichen Landkreis gelegenen Delligsen) eines direkt vom Einsatzort in die Interventionsklinik transportierten Patienten betrug $34 \mathrm{~km}$ und wurde in 48 min zurückgelegt. Aus der Gruppe der Sekundärtransporte hatten sich 2 Patienten aus dem Raum Gronau zunächst in der näher gelegenen Nicht-Interventionsklinik vorgestellt, wobei 1 Patient per RTW allerdings ohne Notarztbegleitung transportiert worden war. Die übrigen 5 Patienten stellten sich zunächst in der Nicht-Interventionsklinik Alfeld vor, wobei 2 dieser Patienten aus dem Alfelder Stadtgebiet stammten. Die 3 übrigen Patienten legten einen weiteren Anfahrtsweg in die Nicht-Interventionsklinik zurück. Hiervon wurde ein Patient aus Delligsen zunächst notarztbegleitet in die näher gelegene Nicht-Interventionsklinik nach Alfeld transportiert. Am Beispiel eines weiteren Patienten aus dem Ort Sibbesse, welcher geographisch auf halbem Weg zwischen der Stadt Hildesheim und Alfeld liegt, wird deutlich, dass eine erhebliche Zeitverzögerung dadurch entstanden ist, dass sich dieser zunächst in die Nicht-Interventionsklinik nach Alfeld begab. Von den 4 Patienten, die sich ohne vorherigen Notarztkontakt in der Interventionsklinik vorstellten, stammte die Hälfte aus dem Stadtgebiet, die anderen zwei aus dem nördlichen Landkreis. Insgesamt fällt auf, dass der südöstliche Landkreis ausgespart ist (Abbildung 6).

Im II. Quartal stammte die Mehrzahl der Patienten aus dem nördlichen Teil des Landkreises, wobei etwa $27 \%$ der notarztbegleiteten Patienten aus der Gruppe der Primärtransporte aus dem Stadtgebiet in die Interventionsklinik transportiert wurden. Die längste Transportzeit eines aus dem 37 km entfernten Freden (südlicher Landkreis) stammenden Patienten betrug 46 min. Von den insgesamt 5 Patienten aus der Gruppe der Sekundärtransporte waren 3 Patienten zunächst in die Nicht-Interventionsklinik nach Alfeld transportiert worden. Davon war ein Patient per RTW, ein weiterer per Notarzt aus dem südlich von Alfeld gelegenen Delligsen begleitet worden. Ein anderer Patient wurde aus Adenstedt, welches zwischen Alfeld und Hildesheim liegt, per RTW zunächst nach Alfeld verbracht. Von den zwei Patienten, die als Sekundärtransport aus der Nichtinterventionsklinik in Gronau zugewiesen wurden, war eine Patientin zuvor per RTW aus dem westlich der Landkreisgrenze gelegenen Bad Salzhemmendorf und ein weiterer Patient aus dem Stadtgebiet Gronau per Notarzt in die dortige Nicht-Interventionsklinik transportiert worden. 
Lediglich ein Patient, der aus dem nordwestlichen Stadtgebiet stammte, stellte sich ohne vorherigen Notarztkontakt in der Interventionsklinik vor. Wiederum wurde aus dem südöstlichen Landkreis kein Patient rekrutiert (Abbildung 7).

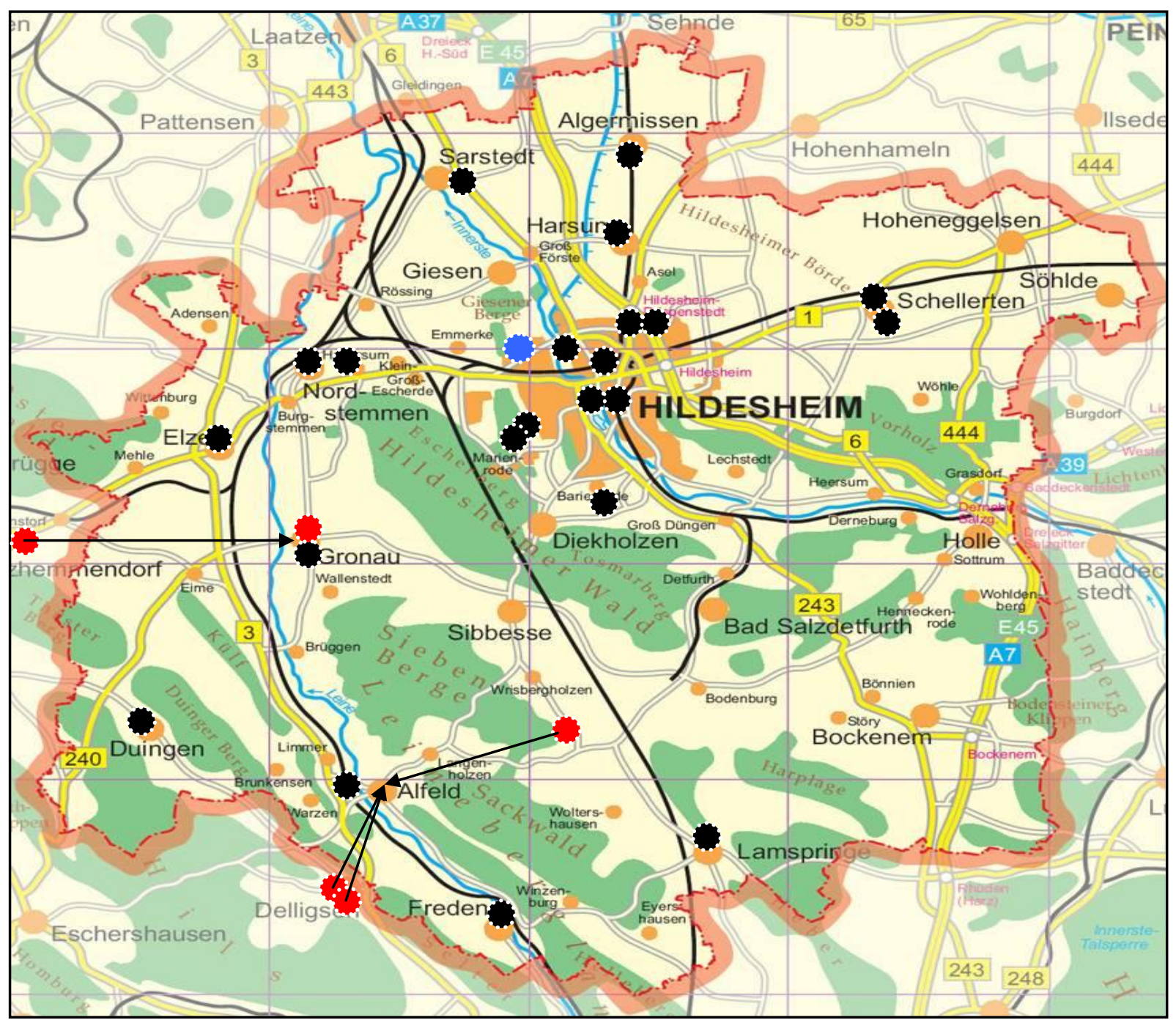

Abbildung 7: Herkunft Patienten II. Quartal (schwarz=Primärtransport, rot=Sekundärtransport, blau=Selbsteinweisung)

Im III. Quartal stammten etwa $26 \%$ der per Notarzt direkt in die Interventionsklinik transportierten Patienten aus dem Stadtgebiet Hildesheim. Erstmals gelangten alle Patienten aus der Region Alfeld direkt notarztbegleitet in die Interventionsklinik. Der insgesamt weiteste Anfahrtsweg an der Nicht-Interventionsklinik in Alfeld vorbei betrug $34 \mathrm{~km}$ und wurde in 48 min zurückgelegt (Abbildung 8). 
Im diesem Quartal wurde erstmals ein Patient aus den nördlich-östlich an den Landkreis Hildesheim grenzenden Regionen von dem dort zuständigen Notarzteinsatzfahrzeug einer nicht am Herzinfarktnetz partizipierenden Klinik direkt in die nahe gelegene Interventionsklinik nach Hildesheim transportiert.

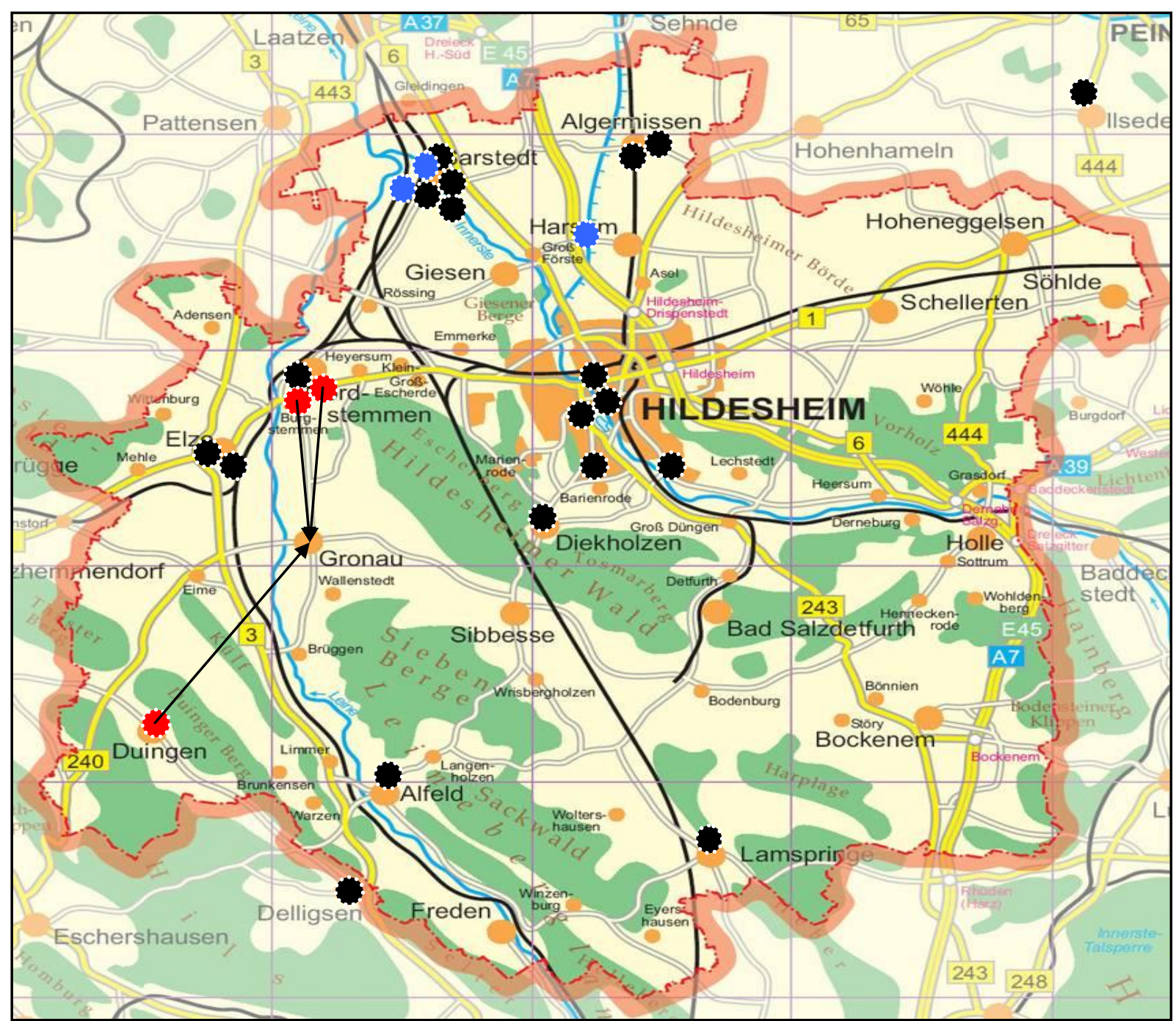

Abbildung 8: Herkunft Patienten III. Quartal (schwarz=Primärtransport, rot=Sekundärtransport, blau=Selbsteinweisung)

In der Gruppe der Sekundärtransporte stammten 2 der 3 Patienten aus dem nördlich von Gronau gelegenen Nordstemmen, von denen einer ebenso wie ein weiterer aus dem südwestlich von Gronau gelegenen Duingen per RTW in die Nicht-Interventionsklinik transportiert wurde. Der andere aus Nordstemmen stammende Patient hatte sich ohne vorherigen Notarztkontakt in der Notaufnahme in der Nicht-Interventionsklinik in Gronau vorgestellt. 
Äquivalent dazu hatten sich im III. Quartal insgesamt 3 Patienten aus dem nördlichen Landkreis selbsttätig in der Notaufnahme der Interventionsklinik vorgestellt.

Im IV. Quartal kamen insgesamt lediglich 26\% der Patienten aus der Gruppe der Primärtransporte aus dem Stadtgebiet. Neben 2 Patienten, die wiederum aus den nordöstlich an den Landkreis grenzenden Regionen stammten, wurden erstmals auch Patienten aus dem südöstlichen Landkreis rekrutiert. Die weiteste Anfahrt im IV. Quartal und auch insgesamt im gesamten Beobachtungsraum betrug $38 \mathrm{~km}$ aus dem an der südlichen Spitze des Landkreises gelegenen Esbeck (Freden), und wurde in nur 39 min zurückgelegt (Abbildung 9).

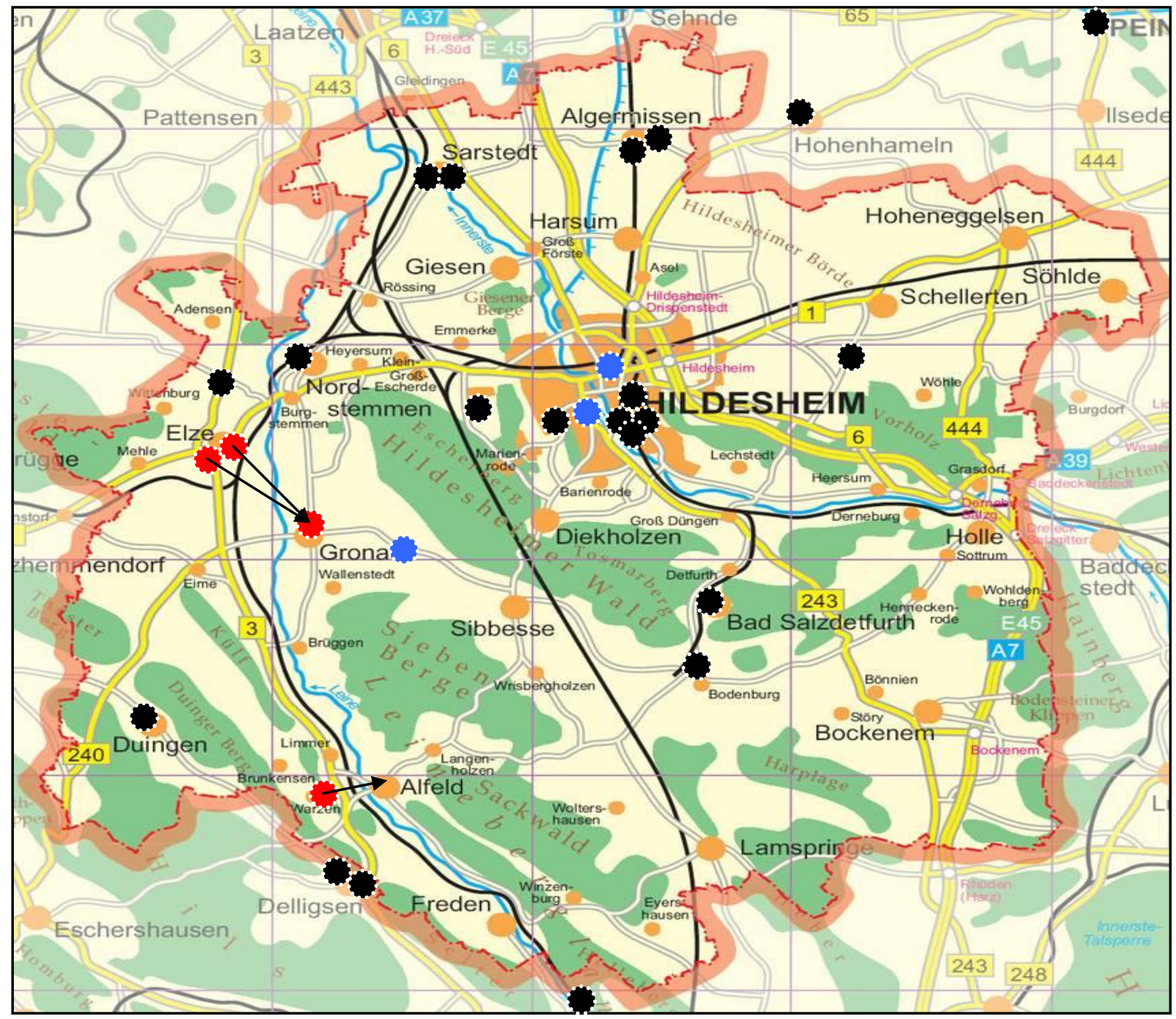

Abbildung 9: Herkunft Patienten IV. Quartal (schwarz=Primärtransport, rot=Sekundärtransport, blau=Selbsteinweisung) 
Die Gruppe der Sekundärtransporte setzte sich aus insgesamt 4 Patienten zusammen, von denen sich 2 Patienten aus Elze stammend ohne vorherigen Notarztkontakt in der Notaufnahme der Nicht-Interventionsklinik in Gronau vorstellten. Ein weiterer Patient befand sich zum Zeitpunkt des Symptombeginns stationär im Krankenhaus in Gronau. Der aus der Nicht-Interventionsklinik in Alfeld in die Interventionsklinik nach Hildesheim verlegte Patient war zuvor per RTW aus dem westlich von Alfeld gelegenen Warzen in die nahe gelegene Nicht-Interventionsklinik transportiert worden.

Innerhalb der Gruppe der Selbstvorsteller stammte ein Patient aus dem Stadtgebiet Hildesheim, ein weiterer befand sich zum Zeitpunkt des Symptombeginns stationär in der Interventionsklinik. Der aus dem nahe an Gronau gelegenen Eitzum stammende Patient hatte sich direkt in der Notaufnahme der Interventionsklinik vorgestellt (Abbildung 9). 


\subsection{Zeitintervalle}

Nachfolgend sind die ermittelten Zeitintervalle tabellarisch als auch grafisch in Säulendiagrammen bzw. Kaplan-Meier-Kurven dargestellt. Die Intervalle sind gegliedert in „Alarmierung bis Erstkontakt“, 5 Unterintervalle der contact-to-balloon Zeit, sowie separat door-to-balloon-Zeit und contact-to-balloon-Zeit. Sie werden jeweils im Quartalsvergleich und in die einzelnen Patientengruppen unterteilt.

\subsubsection{Gesamtgruppe}

Über den Beobachtungszeitraum konnte eine hochsignifikante Reduktion der contact-toballoon- und door-to-balloon Zeiten nachgewiesen werden. Im Durchschnitt verkürzte sich die mittlere door-to-balloon-Zeit von $63 \mathrm{~min}$ im I. Quartal auf $47 \mathrm{im} \mathrm{II.} \mathrm{Quartal,} 37 \mathrm{~min}$ im III. Quartal und 36 min im IV. Quartal (Median: 54, 35, 31 und 26 min; p<0,0001). Die mittlere contact-to-balloon-Zeit betrug im Ausgangsquartal 129 min (Median: $113 \mathrm{~min}$ ) und konnte in den nachfolgenden Quartalen auf 90, 78 und 76 min reduziert werden (Median: 83, 66, 74 min; $\mathrm{p}<0,0001$ ) (Abbildung 10, Tabelle 8).

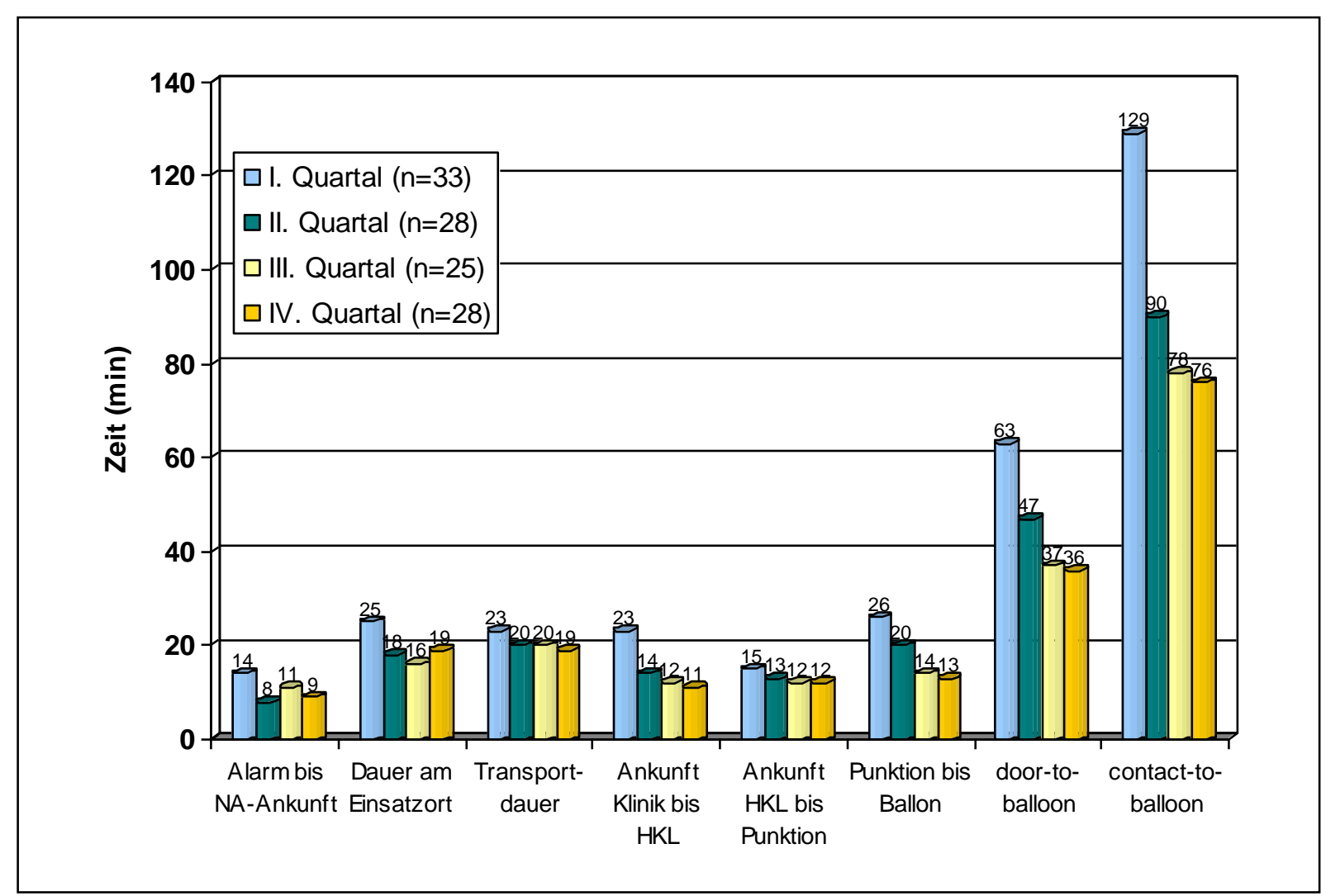

Abbildung 10: Zeitintervalle der Gesamtgruppe im Quartalsvergleich (Mittelwert) 


\begin{tabular}{|c|c|c|c|c|c|}
\hline & $\begin{array}{c}\text { I. } \\
\text { Quartal } \\
(n=33)\end{array}$ & $\begin{array}{c}\text { II. } \\
\text { Quartal } \\
(n=28)\end{array}$ & $\begin{array}{c}\text { III. } \\
\text { Quartal } \\
(n=25)\end{array}$ & $\begin{array}{c}\text { IV. } \\
\text { Quartal } \\
(n=28)\end{array}$ & p-Wert \\
\hline $\begin{array}{r}\text { Symptombeginn bis } \\
\text { Ankunft Interventionsklinik } \\
\text { Mittelwert } \\
\text { Median }\end{array}$ & $\begin{array}{c}159 \pm 121,4 \\
124 \\
\end{array}$ & $\begin{array}{c}182 \pm 117,6 \\
126 \\
\end{array}$ & $\begin{array}{c}187 \pm 133,2 \\
132 \\
\end{array}$ & $\begin{array}{c}158 \pm 154,4 \\
115 \\
\end{array}$ & n.s. \\
\hline $\begin{array}{r}\text { Alarm bis Notarztankunft } \\
\text { Mittelwert } \\
\text { Median }\end{array}$ & $\begin{array}{c}14 \pm 19,5 \\
10 \\
\end{array}$ & $\begin{array}{c}8 \pm 4,5 \\
8 \\
\end{array}$ & $\begin{array}{c}11 \pm 10,1 \\
8 \\
\end{array}$ & $\begin{array}{c}9 \pm 4,7 \\
10 \\
\end{array}$ & n.s. \\
\hline $\begin{array}{r}\text { Aufenthalt am Einsatzort } \\
\text { Mittelwert } \\
\text { Median }\end{array}$ & $\begin{array}{c}25 \pm 10,1 \\
25 \\
\end{array}$ & $\begin{array}{c}18 \pm 10,1 \\
17 \\
\end{array}$ & $\begin{array}{c}16 \pm 8,7 \\
15 \\
\end{array}$ & $\begin{array}{c}19 \pm 7,6 \\
19 \\
\end{array}$ & 0.0644 \\
\hline \begin{tabular}{|r|} 
Transportdauer \\
\\
\\
Mittelwert \\
Median
\end{tabular} & $\begin{array}{c}23 \pm 13,1 \\
23\end{array}$ & $\begin{array}{c}20 \pm 10,7 \\
20\end{array}$ & $\begin{array}{c}20 \pm 11,0 \\
17\end{array}$ & $\begin{array}{c}19 \pm 9,7 \\
16\end{array}$ & n.s. \\
\hline 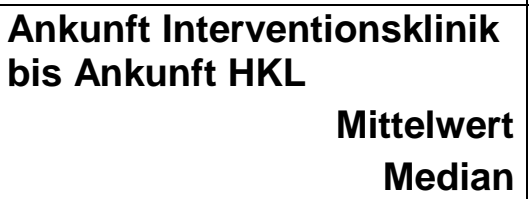 & $\begin{array}{c}23 \pm 22,0 \\
14\end{array}$ & $\begin{array}{c}14 \pm 20,2 \\
2\end{array}$ & $\begin{array}{c}12 \pm 17,6 \\
3\end{array}$ & $\begin{array}{c}11 \pm 16,2 \\
3\end{array}$ & 0.0169 \\
\hline $\begin{array}{l}\text { Ankunft HKL bis Punktion } \\
\text { (Vorbereitung HKL) } \\
\text { Mittelwert } \\
\text { Median }\end{array}$ & $\begin{array}{c}15 \pm 4,1 \\
14\end{array}$ & $\begin{array}{c}13 \pm, 3,5 \\
12\end{array}$ & $\begin{array}{c}12 \pm 4,0 \\
11\end{array}$ & $\begin{array}{c}12 \pm 3,9 \\
12\end{array}$ & 0.0075 \\
\hline $\begin{array}{l}\text { Punktion bis Ballon } \\
\begin{array}{lr}\text { (Therapie HKL) } \\
\text { Mittelwert } \\
\text { Median }\end{array} \\
\end{array}$ & $\begin{array}{c}26 \pm 14,4 \\
21\end{array}$ & $\begin{array}{c}20 \pm 11,0 \\
18\end{array}$ & $\begin{array}{c}14 \pm 5,8 \\
12 \\
\end{array}$ & $\begin{array}{c}13 \pm 6,6 \\
11\end{array}$ & $<0.0001$ \\
\hline $\begin{array}{r}\text { "door-to-balloon" } \\
\qquad \begin{array}{r}\text { Mittelwert } \\
\text { Median }\end{array} \\
\end{array}$ & $\begin{array}{c}63 \pm 30,1 \\
54 \\
\end{array}$ & $\begin{array}{c}47 \pm 29,2 \\
35 \\
\end{array}$ & $\begin{array}{c}37 \pm 17,1 \\
31 \\
\end{array}$ & $\begin{array}{c}36 \pm 22,5 \\
26 \\
\end{array}$ & $<0.0001$ \\
\hline $\begin{array}{r}\text { "contact-to-balloon" } \\
\text { Mittelwert } \\
\text { Median }\end{array}$ & $\begin{array}{c}129 \pm 50,6 \\
113\end{array}$ & $\begin{array}{c}90 \pm 31,2 \\
83\end{array}$ & $\begin{array}{c}78 \pm 31,3 \\
66\end{array}$ & $\begin{array}{c}76 \pm 21,3 \\
74\end{array}$ & $<0.0001$ \\
\hline $\begin{aligned} \text { "contact-to-balloon" no.(\%) } \\
<90 \mathrm{~min} \\
<120 \mathrm{~min}\end{aligned}$ & $\begin{array}{c}7(21,2) \\
18(54,5)\end{array}$ & $\begin{array}{l}17(60,7) \\
25(89,3)\end{array}$ & $\begin{array}{l}19(76,0) \\
22(88,0)\end{array}$ & $\begin{array}{l}22(78,6) \\
28(100)\end{array}$ & $\begin{array}{l}<0.0001 \\
<0.0001\end{array}$ \\
\hline
\end{tabular}

Tabelle 8: Zeitintervalle der Rettungs- und Therapiekette in der Gesamtgruppe im Quartalsvergleich (min) 


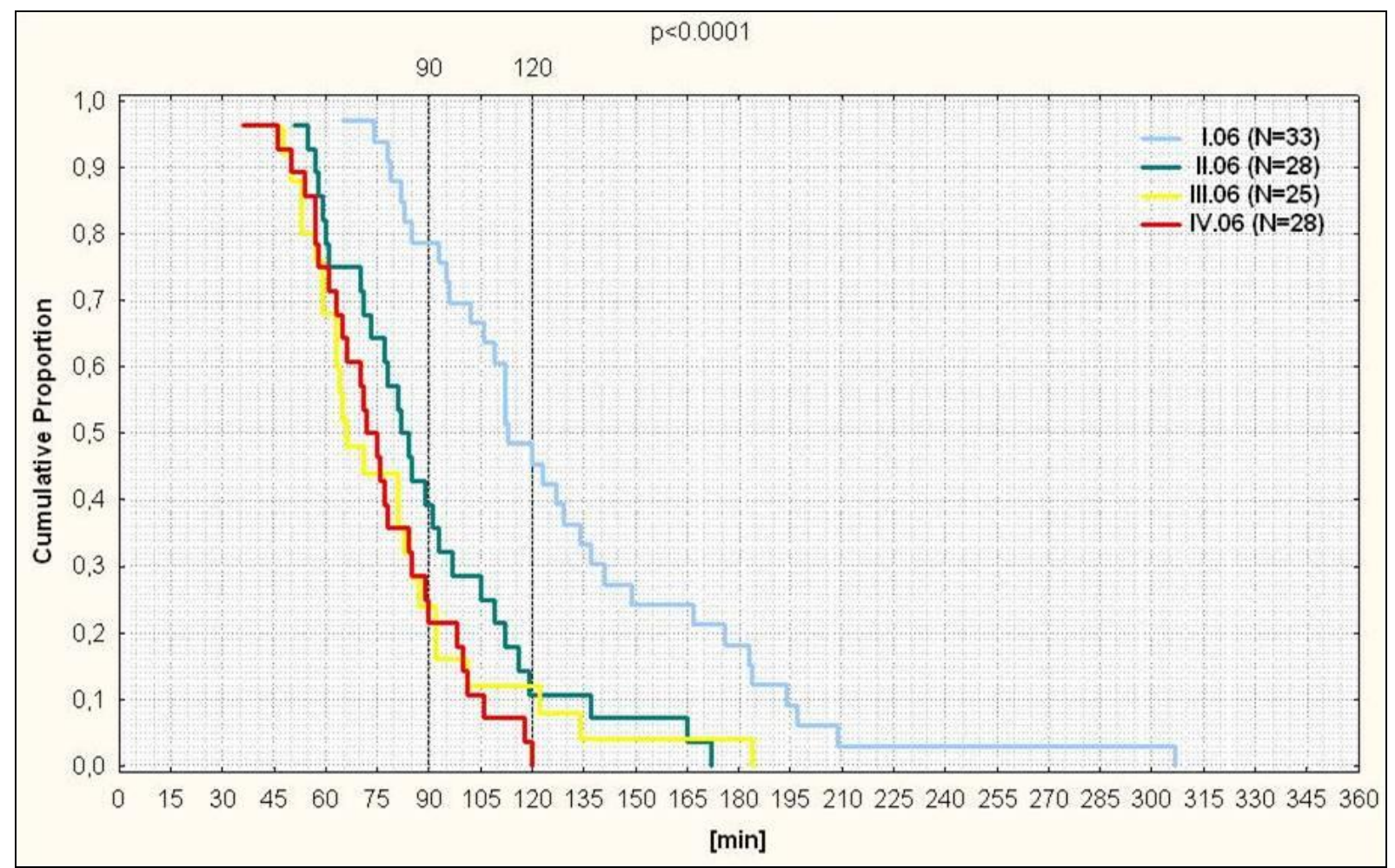

Abbildung 11: Kaplan-Meier-Kurve: contact-to-balloon-Zeit Gesamtgruppe im Quartalsvergleich

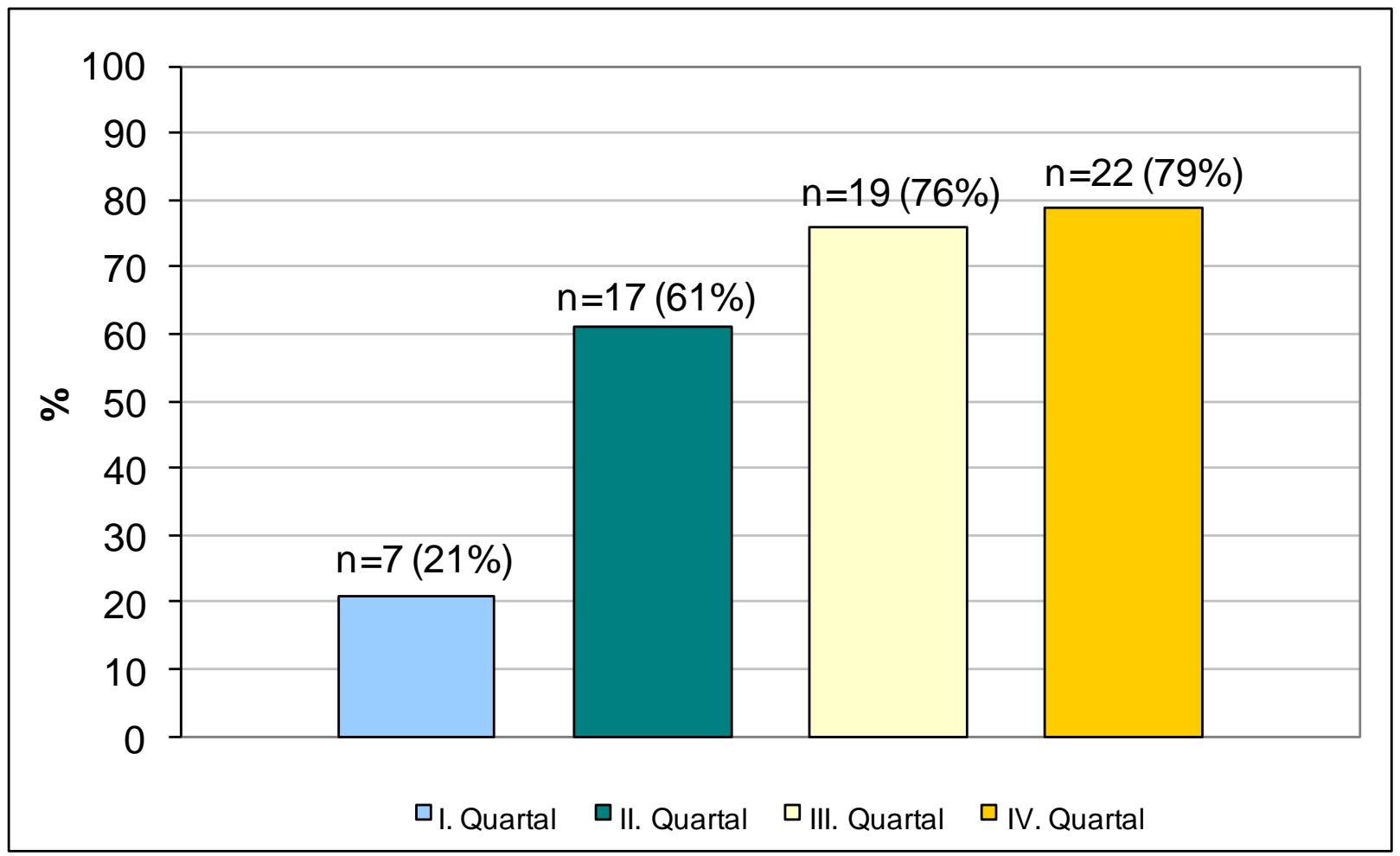

Abbildung 12: Gesamtgruppe: Anteil der Patienten mit contact-to-balloon-Zeit < 90min 
Dadurch stieg der prozentuale Anteil derjenigen Patienten, deren contact-to-balloon-Zeit unter 90 min lag hochsignifikant von 21\% im I. Quartal auf 61\% im II. Quartal, 76\% im III. Quartal und 79\% im IV. Quartal (Abbildung 11, Abbildung 12). Im IV. Quartal wurde bei allen Patienten eine contact-to-balloon-Zeit unter 120 min erzielt (Tabelle 8).

Entsprechend erhöhte sich die Anzahl der Patienten mit einer door-to-balloon-Zeit unter 30 min hochsignifikant von 9\% im I. Quartal auf 36\% im II. Quartal, 48\% im III. Quartal und 57\% im IV. Quartal (Abbildung 13).

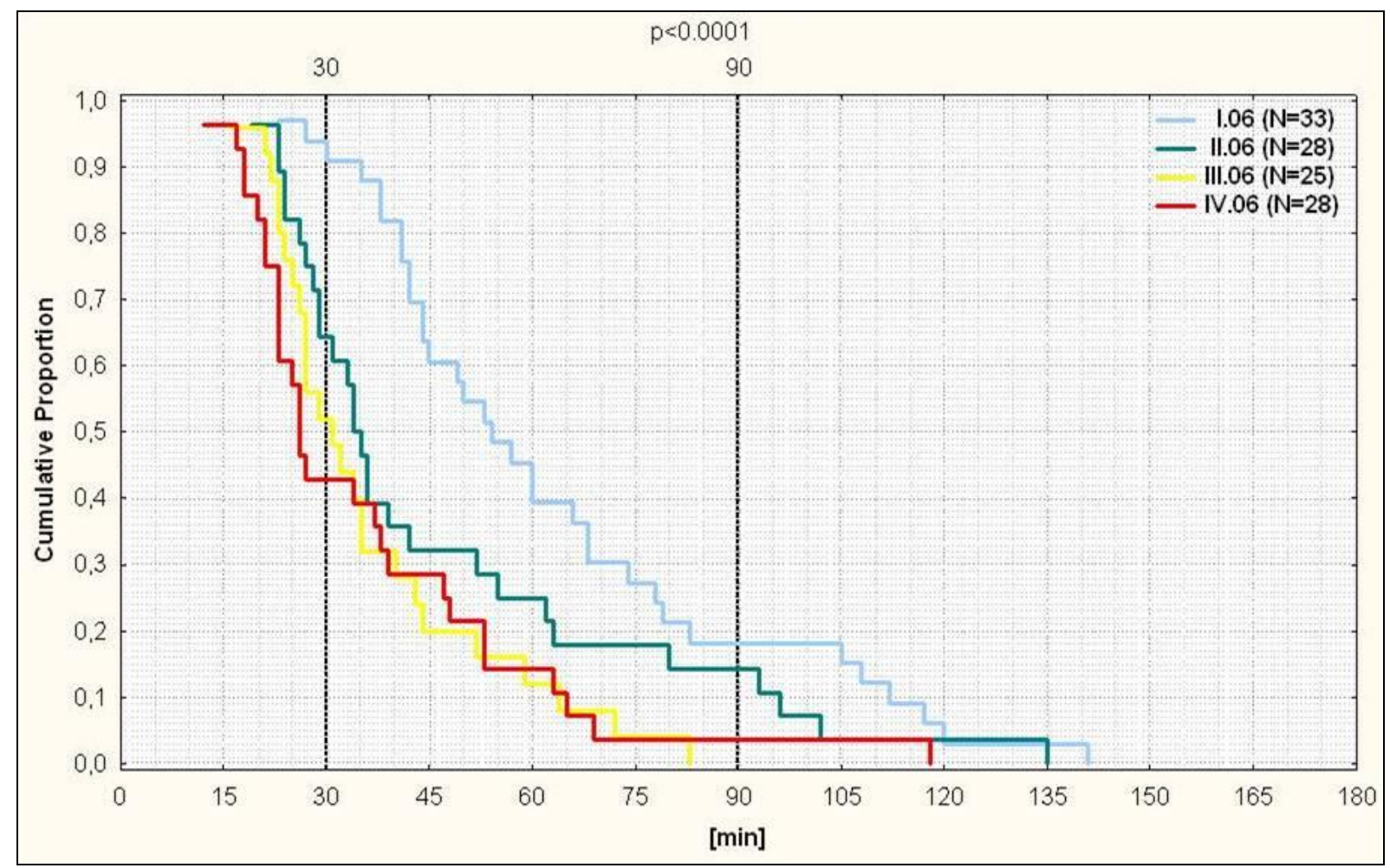

Abbildung 13: Kaplan-Meier-Kurve: door-to-balloon-Zeit Gesamtgruppe im Quartalsvergleich

Die Zeitgewinne wurden im Wesentlichen beim Aufenthalt des Notarztes vor Ort, dem Aufenthalt des Patienten in der Notaufnahme sowie der Vorbereitung und Therapie im HKL erzielt. Die Aufenthaltsdauer des Notarztes am Einsatzort reduzierte sich von im Mittel 25 min im I. Quartal auf 18 min im II. Quartal und 16 min im III. Quartal (Median: 25, 17, 15 min), so dass eine Einsatzzeit von weniger als $20 \mathrm{~min}$ im I. Quartal bei $42 \%$ der Patienten, im II. Quartal bei 64\% und im III. Quartal bei 76\% der Patienten vorlag. Im IV. Quartal wurde mit einem Durchschnittswert von 19 min allerdings wieder ein Anstieg der Aufenthaltsdauer am Noteinsatzort dokumentiert (Median: 19 min; p=0,0644). 
Hervorzuheben ist insbesondere die mittlere Verkürzung des Aufenthaltes der Patienten in der Notaufnahme von im I. Quartal 23 min auf 14 min im II. Quartal, 12 min im III. Quartal und 11 min im IV. Quartal (Median: 14, 2, 3, 3 min; p=0,017), so dass sich ein Anstieg des Anteils derjenigen Patienten, die bereits 5 min nach Ankunft in der Interventionsklinik das HK-Labor (door-to-cath) erreicht hatten, von 33\% im I. Quartal auf 57\% im II. Quartal, 64\% im III. Quartal und 68\% im IV. Quartal verzeichnen ließ (Abbildung 14).

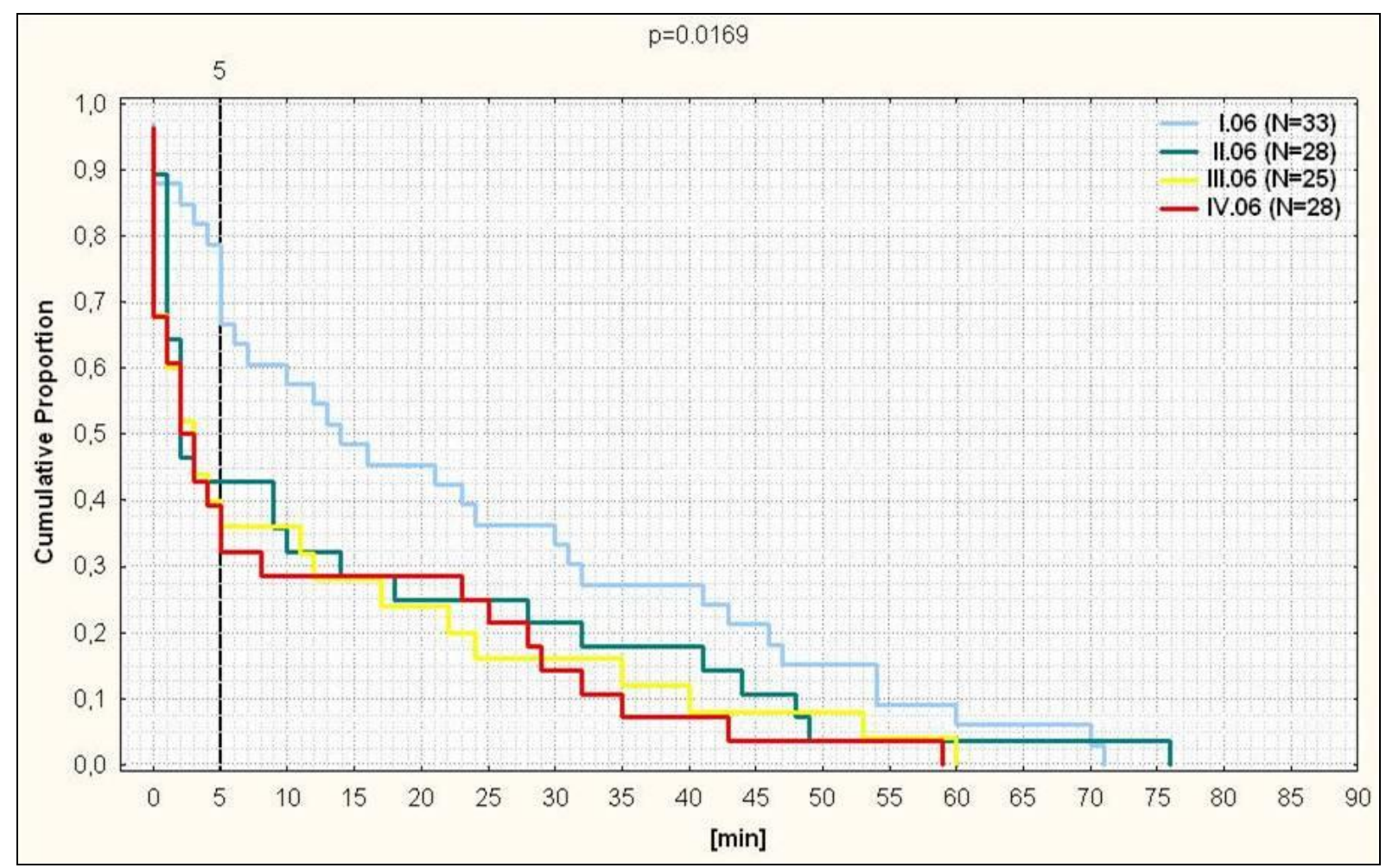

Abbildung 14: Kaplan-Meier-Kurve: door-to-cath-Zeit Gesamtgruppe im Quartalsvergleich

Die Vorbereitungszeit im HK-Labor verringerte sich von im Mittel 15 min im I. Quartal auf 13 min im II. Quartal und je 12 min im III. und IV. Quartal (Median: 14, 12, 11 und 12 min; $\mathrm{p}=0.0075)$. Im Quartalsvergleich verkürzte sich die mittlere Interventionsdauer im HK-Labor (Punktion bis Ballon) von $26 \mathrm{~min}$ im I. Quartal auf $20 \mathrm{~min}$ im II. Quartal, $14 \mathrm{~min}$. im III. Quartal und 13 min im IV. Quartal (Median von 21 auf 18, 12 und $11 \mathrm{~min}$; p<0.0001). Die Zeit von Alarmierung bis Ankunft des Notarztes war mit Zeiten zwischen im Mittel 8 bis 14 min im Quartalsvergleich ebenso wenig signifikant verändert wie die Transportdauer (im Mittel 19 bis $23 \mathrm{~min}$ ). Die Prähospitalzeit (Symptombeginn bis Ankunft in der Interventionsklinik) betrug im gesamten Beobachtungszeitraum durchschnittlich 158 bis 187 in (Median: 115 bis 132 min) und wies im Quartalsvergleich ebenfalls keinen signifikanten Unterschied auf (Tabelle 8). 


\subsubsection{Patienten mit Primärtransport}

Ähnlich wie in der Gesamtgruppe konnte in der Gruppe der Primärtransporte, die den Hauptanteil der Gesamtgruppe darstellte (Tabelle 8), ebenfalls eine hochsignifikante Verkürzung der Reperfusionszeiten im Quartalsvergleich dokumentiert werden. Die mittlere contact-to-balloon-Zeit verkürzte sich hochsignifikant von $110 \mathrm{~min}$ im I. Quartal auf $84 \mathrm{~min}$ im II. Quartal, 71 min im III. Quartal und 69 min im IV. Quartal (Median: 111, 78, 65 und 70 min, $\mathrm{p}<0,0001)$. Die door-to-balloon-Zeit lag im Ausgangsquartal bei im Mittel 63 min, im II. Quartal gelang eine Verkürzung auf $47 \mathrm{~min}$ mit schließlich Halbierung auf $32 \mathrm{~min}$ im III. und IV. Quartal (Median: 56, 32, 27, $26 \mathrm{~min}$; p<0,0001). Dies ließ sich wiederum im Wesentlichen auf einen signifikant verkürzten Aufenthalt am Einsatzort und in der Notaufnahme als auch durch die verringerte Interventionsdauer zurückführen. So konnte die mittlere Aufenthaltsdauer des Patienten in der Notaufnahme von 24 min im I. Quartal auf 16 min im II. Quartal, 9 min im III. Quartal und 8 min im IV. Quartal reduziert werden (Median: 19, 6, 3, 2 min; $\mathrm{p}<0,0077$ ) (Abbildung 15, Tabelle 9).

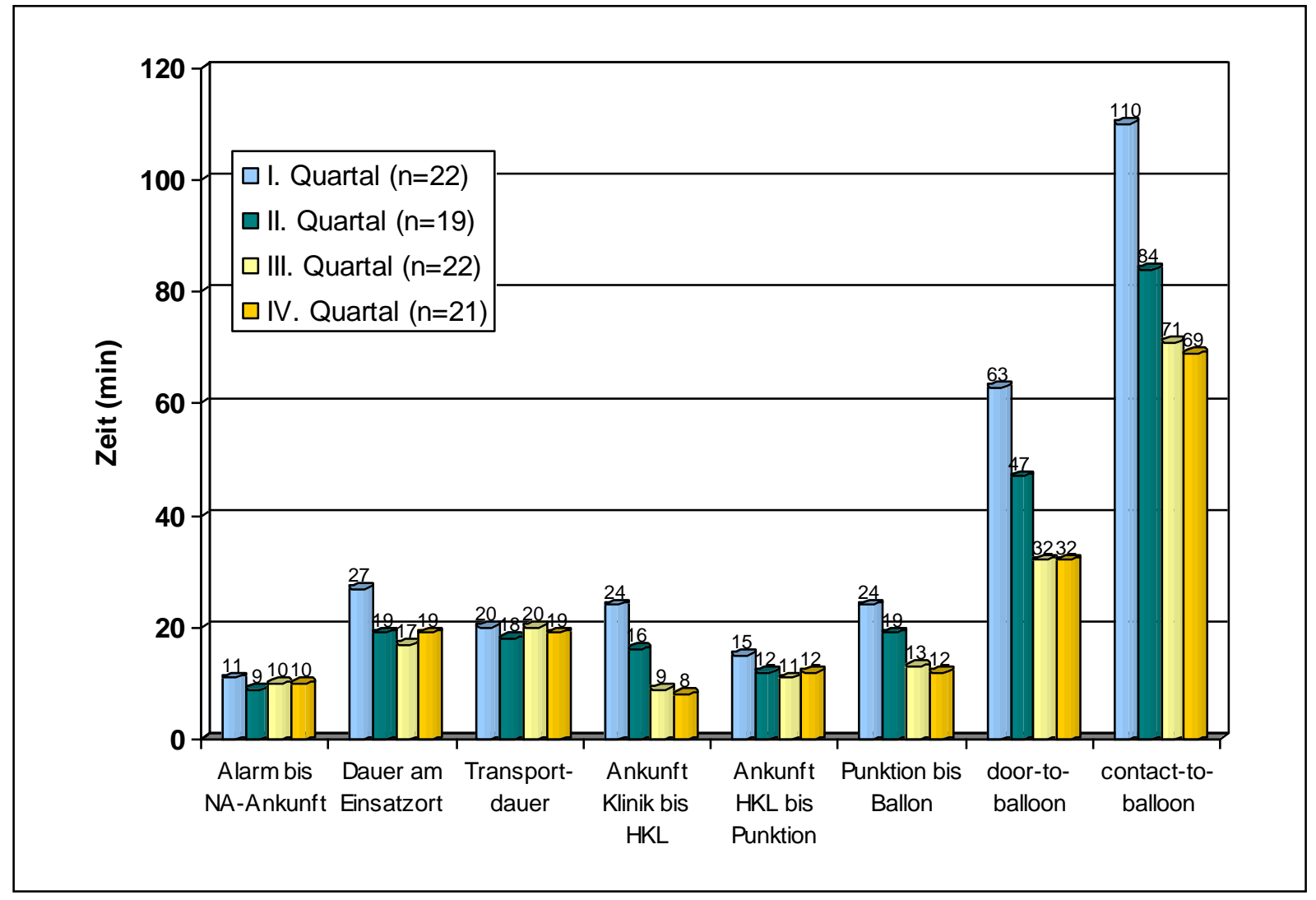

Abbildung 15: Zeitintervalle Gruppe der Primärtransporte im Quartalsvergleich (Mittelwert) 


\begin{tabular}{|c|c|c|c|c|c|}
\hline & $\begin{array}{c}\text { I. } \\
\text { Quartal } \\
(n=22)\end{array}$ & $\begin{array}{c}\text { II. } \\
\text { Quartal } \\
(n=19)\end{array}$ & $\begin{array}{c}\text { III. } \\
\text { Quartal } \\
(\mathrm{n}=22)\end{array}$ & $\begin{array}{c}\text { IV. } \\
\text { Quartal } \\
(n=21)\end{array}$ & p-Wert \\
\hline $\begin{array}{r}\text { Symptombeginn bis } \\
\text { Ankunft Interventionsklinik } \\
\text { Mittelwert } \\
\text { Median }\end{array}$ & $\begin{array}{c}139 \pm 133,8 \\
114 \\
\end{array}$ & $\begin{array}{c}157 \pm 110,3 \\
112 \\
\end{array}$ & $\begin{array}{c}145 \pm 64,6 \\
111 \\
\end{array}$ & $\begin{array}{c}166 \pm 172,3 \\
115 \\
\end{array}$ & n.s. \\
\hline $\begin{array}{r}\text { Alarm bis Notarztankunft } \\
\text { Mittelwert } \\
\text { Median }\end{array}$ & $\begin{array}{c}11 \pm 4,1 \\
11 \\
\end{array}$ & $\begin{array}{c}9 \pm 4,3 \\
10 \\
\end{array}$ & $\begin{array}{c}10 \pm 5,3 \\
8 \\
\end{array}$ & $\begin{array}{c}10 \pm 4,3 \\
11 \\
\end{array}$ & n.s. \\
\hline $\begin{array}{r}\text { Aufenthalt am Einsatzort } \\
\text { Mittelwert } \\
\text { Median }\end{array}$ & $\begin{array}{c}27 \pm 10,2 \\
26 \\
\end{array}$ & $\begin{array}{c}19 \pm 10,0 \\
18 \\
\end{array}$ & $\begin{array}{c}17 \pm 8,7 \\
15 \\
\end{array}$ & $\begin{array}{c}19 \pm 7,0 \\
20 \\
\end{array}$ & 0,013 \\
\hline \begin{tabular}{|r|} 
Transportdauer \\
\\
\\
Mittelwert \\
Median
\end{tabular} & $\begin{array}{c}20 \pm 13,8 \\
20\end{array}$ & $\begin{array}{c}18 \pm 10,3 \\
16\end{array}$ & $\begin{array}{c}20 \pm 11,7 \\
17\end{array}$ & $\begin{array}{c}19 \pm 10,1 \\
15\end{array}$ & n.s. \\
\hline 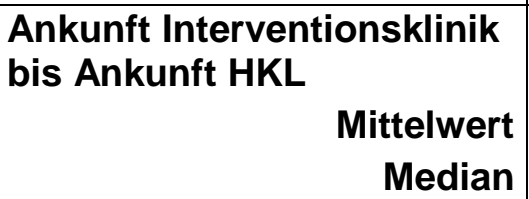 & $\begin{array}{c}24 \pm 20,6 \\
19\end{array}$ & $\begin{array}{c}16 \pm 20,8 \\
6\end{array}$ & $\begin{array}{c}9 \pm 14,6 \\
3\end{array}$ & $\begin{array}{c}8 \pm 11,8 \\
2\end{array}$ & 0,0077 \\
\hline $\begin{array}{l}\text { Ankunft HKL bis Punktion } \\
\text { (Vorbereitung HKL) } \\
\text { Mittelwert } \\
\text { Median }\end{array}$ & $\begin{array}{c}15 \pm 4,6 \\
15 \\
\end{array}$ & $\begin{array}{c}12 \pm 2,4 \\
12 \\
\end{array}$ & $\begin{array}{c}11 \pm 3,3 \\
11 \\
\end{array}$ & $\begin{array}{c}12 \pm 3,3 \\
12 \\
\end{array}$ & 0,0574 \\
\hline $\begin{array}{l}\text { Punktion bis Ballon } \\
\begin{array}{lr}\text { (Therapie HKL) } \\
\text { Mittelwert } \\
\text { Median }\end{array} \\
\end{array}$ & $\begin{array}{c}24 \pm 12,9 \\
20\end{array}$ & $\begin{array}{c}19 \pm 11,8 \\
17\end{array}$ & $\begin{array}{c}13 \pm 4,1 \\
12 \\
\end{array}$ & $\begin{array}{c}12 \pm 5,3 \\
11 \\
\end{array}$ & 0,0002 \\
\hline $\begin{array}{r}\text { "door-to-balloon" } \\
\qquad \begin{array}{r}\text { Mittelwert } \\
\text { Median }\end{array} \\
\end{array}$ & $\begin{array}{c}63 \pm 27,9 \\
56 \\
\end{array}$ & $\begin{array}{c}47 \pm 30,4 \\
32 \\
\end{array}$ & $\begin{array}{c}32 \pm 12,5 \\
27 \\
\end{array}$ & $\begin{array}{c}32 \pm 14,8 \\
26\end{array}$ & $<0.0001$ \\
\hline $\begin{array}{r}\text { "contact-to-balloon" } \\
\text { Mittelwert } \\
\text { Median }\end{array}$ & $\begin{array}{c}110 \pm 25,1 \\
111 \\
\end{array}$ & $\begin{array}{c}84 \pm 29,1 \\
78\end{array}$ & $\begin{array}{c}71 \pm 20,2 \\
65 \\
\end{array}$ & $\begin{array}{c}69 \pm 17,0 \\
70\end{array}$ & $<0.0001$ \\
\hline $\begin{aligned} \text { "contact-to-balloon" } & \text { no. }(\%) \\
& <90 \mathrm{~min} \\
& <120 \mathrm{~min}\end{aligned}$ & $\begin{array}{c}5(22,7) \\
15(68,2)\end{array}$ & $\begin{array}{l}15(68,2) \\
20(90,9) \\
\end{array}$ & $\begin{array}{l}16(84,2) \\
18(94,7) \\
\end{array}$ & $\begin{array}{c}19(90,5) \\
21(100,0) \\
\end{array}$ & $\begin{array}{c}0,0001 \\
0,007 \\
\end{array}$ \\
\hline
\end{tabular}

Tabelle 9: Zeitintervalle der Rettungs- und Therapiekette in der Gruppe der Primärtransporte im Quartalsvergleich ( $\mathrm{min}$ ) 
Der Anteil der Patienten mit einer contact-to-balloon-Zeit innerhalb von 90 Minuten lag im I. Quartal bei 23\% und konnte nachfolgend auf 68\% im II. Quartal, 84\% im III. Quartal und 91\% im IV. Quartal gesteigert werden ( $\mathrm{p}<0,0001)$ (Abbildung 16).

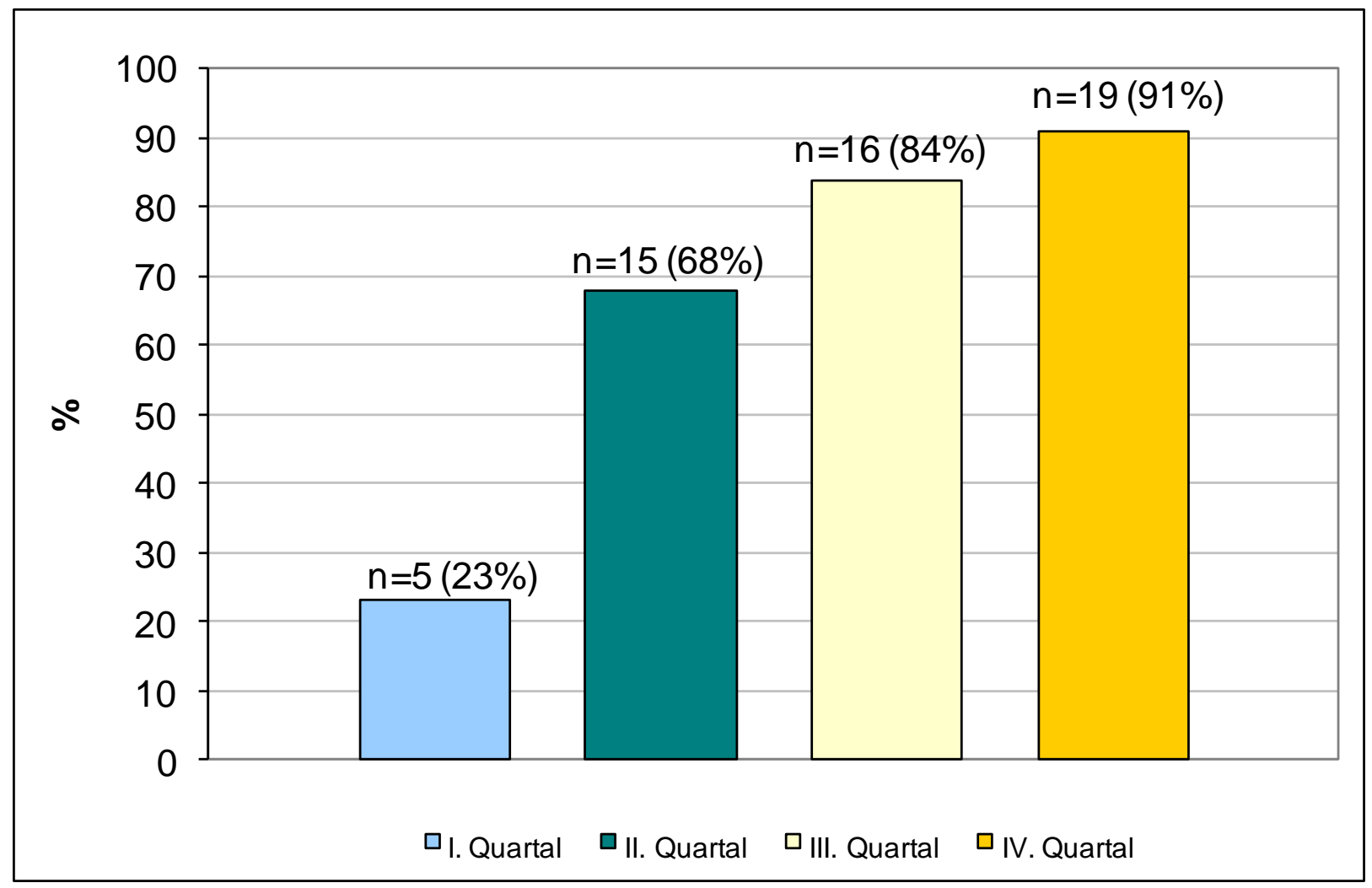

Abbildung 16: Patienten mit Primärtransport: Anteil der Patienten mit einer contact-to-balloon-Zeit $<90$ min 


\subsubsection{Patienten mit Sekundärtransport}

Die Gruppe der Sekundärtransporte machte nur einen kleinen Anteil an der Gesamtgruppe aus, der Anteil war von 21\% im I. Quartal auf 14\% im IV.Quartal rückläufig. Die mittleren contact-to-balloon-Zeiten lagen deutlich über denen in der Gesamt- bzw. Primärtransportgruppe, konnten jedoch im Quartalsvergleich von im Durchschnitt 207 min im I. Quartal auf 116 im II. Quartal, 137 im III. Quartal und 103 min im IV. Quartal signifikant reduziert werden (Median: 194, 109, 134, 104 min; p <0,0073). Die mittlere door-to-balloon-Zeit verkürzte sich im Durchschnitt von 43 im I. Quartal auf 36 im II. Quartal, 30 min im III. Quartal und 26 min im IV. Quartal (Median: 42, 36, 31 und 22 min; p <0,0623). Die Aufenthaltsdauer in der Notaufnahme bei den zuvor angekündigten Patienten betrug bereits im I. Quartal im Durchschnitt nur $4 \mathrm{~min}$ und war im Quartalsvergleich nicht signifikant verschieden. Die Zeitdauer zwischen Vorstellung in der Nicht-Interventionsklinik und Ankunft in der Interventionsklinik (door-to-door) konnte signifikant von im Durchschnitt 154 min im Ausgangsquartal auf 78 min im IV. Quartal gesenkt werden (Median: Von 156 auf 75 min; p <0,0249). Im Gegensatz zum I. Quartal, in dem bei keinem Patienten mit Sekundärtransport eine contact-to-balloon-Zeit unter 120 min erreicht wurde, konnte im IV. Quartal bei allen Patienten innerhalb von $120 \mathrm{~min}$ nach Erstkontakt das verschlossene Infarktgefäß wiedereröffnet werden (Abbildung 17, Tabelle 10).

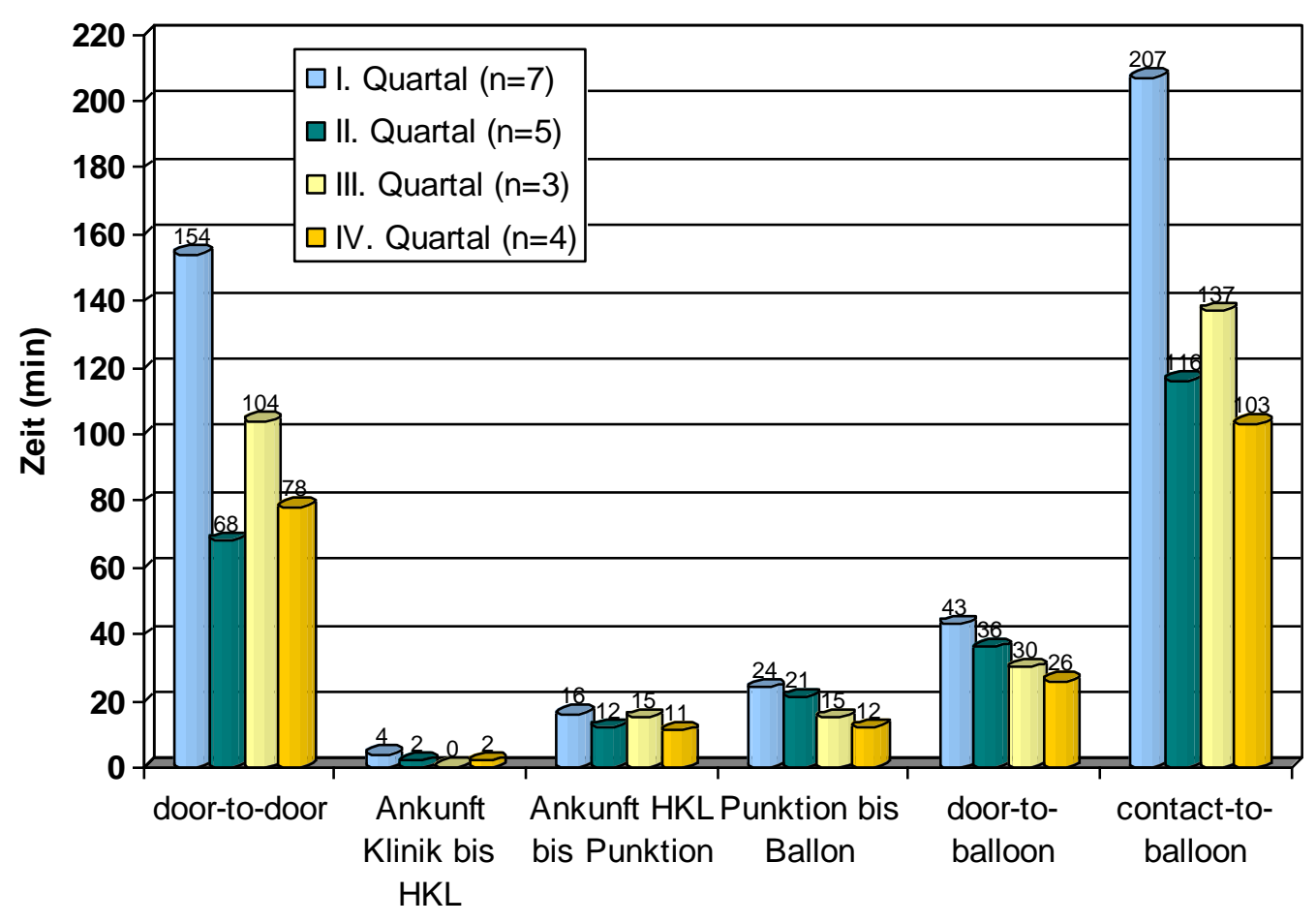

Abbildung 17: Zeitintervalle Gruppe der Patienten mit Sekundärtransport im Quartalsvergleich (Mittelwert) 


\begin{tabular}{|c|c|c|c|c|c|}
\hline & $\begin{array}{c}\text { I. } \\
\text { Quartal } \\
(n=7)\end{array}$ & $\begin{array}{c}\text { II. } \\
\text { Quartal } \\
(n=5)\end{array}$ & $\begin{array}{c}\text { III. } \\
\text { Quartal } \\
(n=3)\end{array}$ & $\begin{array}{c}\text { IV. } \\
\text { Quartal } \\
(n=4)\end{array}$ & p-Wert \\
\hline $\begin{array}{l}\text { Symptombeginn bis Ankunft } \\
\text { Nicht-Interventionsklinik }\end{array}$ & & & & & \\
\hline $\begin{array}{r}\text { Mittelwert } \\
\text { Median }\end{array}$ & $\begin{array}{c}148 \pm 87.7 \\
167\end{array}$ & $\begin{array}{c}205 \pm 111.2 \\
203\end{array}$ & $\begin{array}{c}209 \pm 87.0 \\
209\end{array}$ & $\begin{array}{c}92 \pm 39.5 \\
82\end{array}$ & 0.3222 \\
\hline „door-to-door“ & & & & & \\
\hline $\begin{array}{r}\text { Mittelwert } \\
\text { Median }\end{array}$ & $\begin{array}{c}154 \pm 57.3 \\
156\end{array}$ & $\begin{array}{c}68 \pm 17.5 \\
68\end{array}$ & $\begin{array}{c}104 \pm 44.7 \\
118\end{array}$ & $\begin{array}{c}78 \pm 14.3 \\
75\end{array}$ & 0.0249 \\
\hline $\begin{array}{l}\text { Ankunft Interventionsklinik } \\
\text { bis Ankunft HKL }\end{array}$ & & & & & \\
\hline $\begin{array}{r}\text { Mittelwert } \\
\text { Median }\end{array}$ & $\begin{array}{c}4 \pm 2.5 \\
5\end{array}$ & $\begin{array}{c}2 \pm 1.1 \\
2\end{array}$ & $\begin{array}{c}0 \pm 0.6 \\
0\end{array}$ & $\begin{array}{c}2 \pm 2.2 \\
1\end{array}$ & 0.2374 \\
\hline $\begin{array}{l}\text { Ankunft HKL bis Punktion } \\
\text { (Vorbereitung HKL) }\end{array}$ & & & & & \\
\hline $\begin{array}{r}\text { Mittelwert } \\
\text { Median }\end{array}$ & $\begin{array}{c}16 \pm 3.4 \\
15\end{array}$ & $\begin{array}{c}12 \pm 4.0 \\
14\end{array}$ & $\begin{array}{c}15 \pm 7.4 \\
12\end{array}$ & $\begin{array}{c}11 \pm 2.2 \\
12\end{array}$ & 0.2045 \\
\hline $\begin{array}{l}\text { Punktion bis Ballon } \\
\text { (Therapie HKL) }\end{array}$ & & & & & \\
\hline $\begin{array}{r}\text { Mittelwert } \\
\text { Median }\end{array}$ & $\begin{array}{c}24 \pm 12.1 \\
21\end{array}$ & $\begin{array}{c}21 \pm 7.0 \\
20\end{array}$ & $\begin{array}{c}15 \pm 14.4 \\
7\end{array}$ & $\begin{array}{c}12 \pm 5.7 \\
11\end{array}$ & 0.1894 \\
\hline $\begin{array}{rr}\text { "door-to-balloon" } & \\
& \begin{array}{r}\text { Mittelwert } \\
\text { Median }\end{array}\end{array}$ & $\begin{array}{c}43 \pm 13.0 \\
42\end{array}$ & $\begin{array}{c}36 \pm 1.9 \\
36\end{array}$ & $\begin{array}{c}30 \pm 14.0 \\
31\end{array}$ & $\begin{array}{c}26 \pm 7.7 \\
22\end{array}$ & 0.0623 \\
\hline $\begin{array}{r}\text { "contact-to-balloon" } \\
\text { Mittelwert } \\
\text { Median }\end{array}$ & $\begin{array}{c}207 \pm 45.3 \\
194\end{array}$ & $\begin{array}{c}116 \pm 32.8 \\
109\end{array}$ & $\begin{array}{c}137 \pm 46.1 \\
134\end{array}$ & $\begin{array}{c}103 \pm 14.4 \\
104\end{array}$ & 0.0073 \\
\hline $\begin{aligned} \text { "contact-to-balloon" } & \text { no.(\%) } \\
& <90 \mathrm{~min} \\
& <120 \mathrm{~min}\end{aligned}$ & $\begin{array}{l}0(0.0) \\
0(0.0)\end{array}$ & $\begin{array}{l}1(20.0) \\
4(80.0)\end{array}$ & $\begin{array}{l}0(0.0) \\
1(33.3)\end{array}$ & $\begin{array}{c}1(25.0) \\
4(100.0)\end{array}$ & $\begin{array}{l}0.4676 \\
0.0044\end{array}$ \\
\hline
\end{tabular}

Tabelle 10: Zeitintervalle der Rettungs- und Therapiekette in der Gruppe der Sekundärtransporte im Quartalsvergleich (min)

\subsubsection{Patienten mit Selbsteinweisung}

Die Zeitintervalle in der Gruppe der Selbstvorsteller wurde aufgrund der anteilsmäßig nur sehr kleinen Gruppen ( $\mathrm{n}=4,1,3$ und 3) innerhalb der Gesamtgruppe nicht gesondert grafisch bzw. tabellarisch erfasst. 


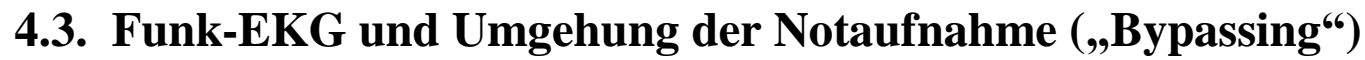

Im gesamten Beobachtungszeitraum wurden 84 der insgesamt 114 in die Untersuchung eingeschlossenen Patienten direkt vom Einsatzort mit dem Notarzt in die Interventionsklinik transportiert. Aus dieser Primärtransportgruppe war bei 64 Patienten (76\%) prähospital ein Funk-EKG abgeleitet und auf die Intensivstation der Interventionsklinik gefaxt worden. In der Gruppe der mittels Funk-EKG angekündigten Patienten konnte eine signifikante Reduktion der contact-to-balloon- und door-to-balloon Zeiten erzielt werden. Die Aufenthaltsdauer des Notarztes am Einsatzort war in beiden Gruppen, mit oder ohne EKG-Registrierung, nicht signifikant verschieden. Im Gegensatz dazu wurde eine signifikante Reduktion des Zeitintervalls zwischen Ankunft in der Interventionsklinik und Ankunft im Herzkatheterlabor von im Mittel 34 min (Median: 30 min) in der Gruppe ohne Funk-EKG auf 8 min (Median: 2 min) in der Gruppe mit Funk-EKG beobachtet ( $<<0,0001)$. Dies führte zu einer deutlichen Verkürzung der door-to-balloon-Zeiten von im Durchschnitt $69 \mathrm{~min}$ in der Gruppe ohne Funk-EKG auf 36 min in der Gruppe mit Funk-EKG (Median: Von 61 auf $29 \mathrm{~min}$ ). Die mittlere contact-to-balloon-Zeit betrug in der Gruppe der unangekündigten Patienten 105 min (Median: $103 \mathrm{~min}$ ) im Vergleich zu $78 \mathrm{~min}$ (Median: $74 \mathrm{~min}$ ) in der Patientengruppe mit Funk-EKG (Abbildung 18).

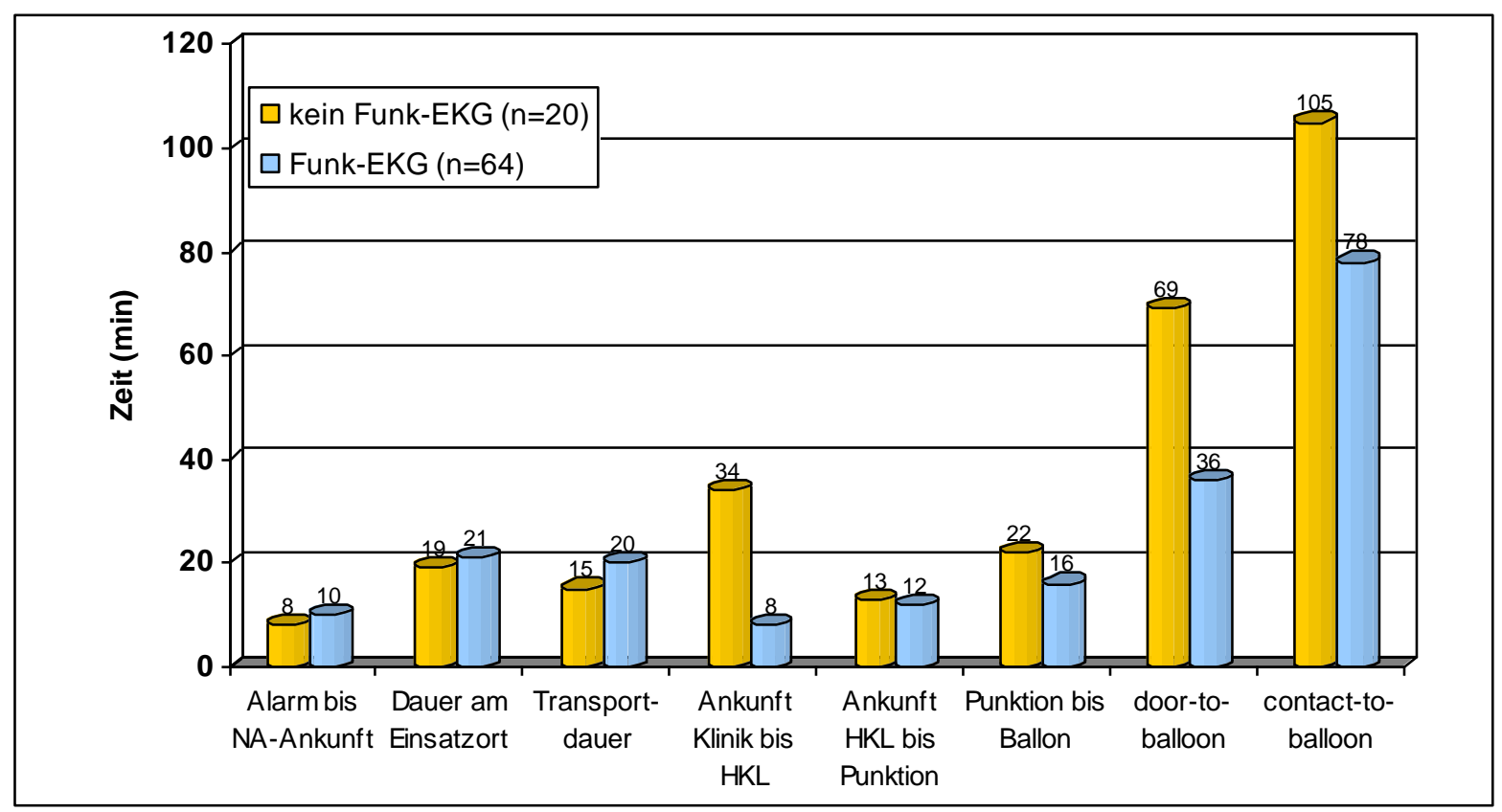

Abbildung 18: Patienten mit Primärtransport: Zeitintervalle der Rettungs- und Therapiekette im Vergleich mit und ohne Funk-EKG (Mittelwert) 
Der Anteil der Patienten aus der Primärtransportgruppe, die mit Übermittlung eines FunkEKG angekündigt worden war, stieg von initial 59\% auf 86\% im II. Quartal und blieb dann konstant bei $74 \%$ und $86 \%$ in den nächsten 2 Quartalen ( $\mathrm{p}=0,1157)$. Im Gegensatz dazu kam es zu einem deutlichen Anstieg des Anteils derjenigen Patienten, die die Notaufnahme umgingen und direkt vom Notarzt an den Interventionskardiologen übergeben wurden (von $23 \%$ im I. Quartal auf 50\%, 68 und 76\% im II, III. und IV. Quartal; p=0,0023) (Abbildung 19).

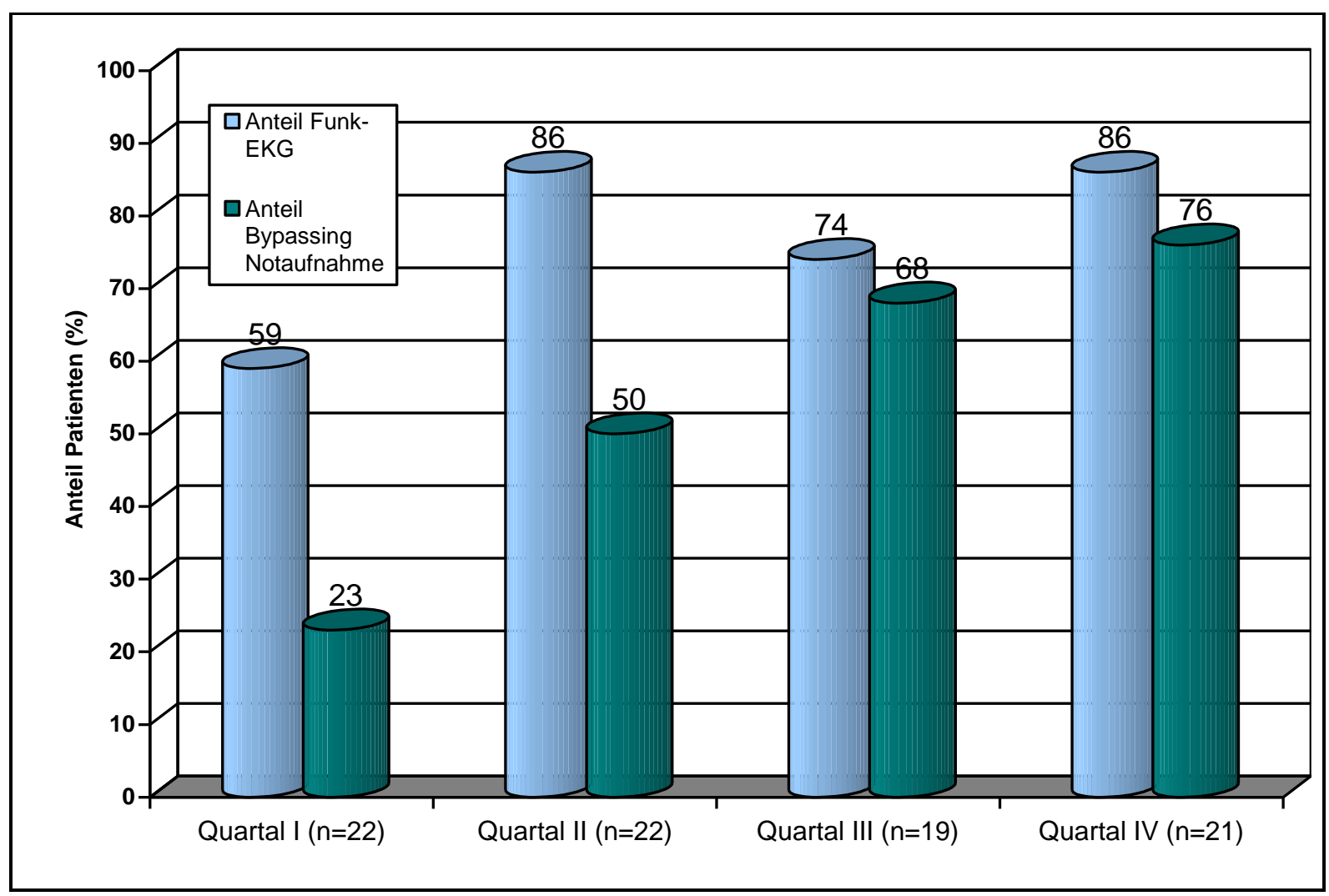

Abbildung 19: Anteil der Patienten mit Funk-EKG und Bypassing der Notaufnahme

Die durchschnittliche door-to-balloon-Zeit in der Primärtransportgruppe ohne Funk-EKG $(\mathrm{n}=20)$ betrug 69 min (Median: 61min) und unterschied sich nicht signifikant von der Gruppe der Primärtransporte mit Funk-EKG ohne Bypassing der Notaufnahme (n=19; Mittelwert: 56 min, Median: 53 min; p=0,2). Dagegen wies die Gruppe der Primärtransportpatienten mit Funk-EKG, die die Notaufnahme umgingen und direkt dem Interventionskardiologen im Herzkatheterlabor übergeben wurden $(n=45)$, im Vergleich eine signifikante Reduktion der door-to-balloon-Zeiten auf (Mittelwert: 27 min, Median: 26 min; p<0,0001) (Abbildung 20). 


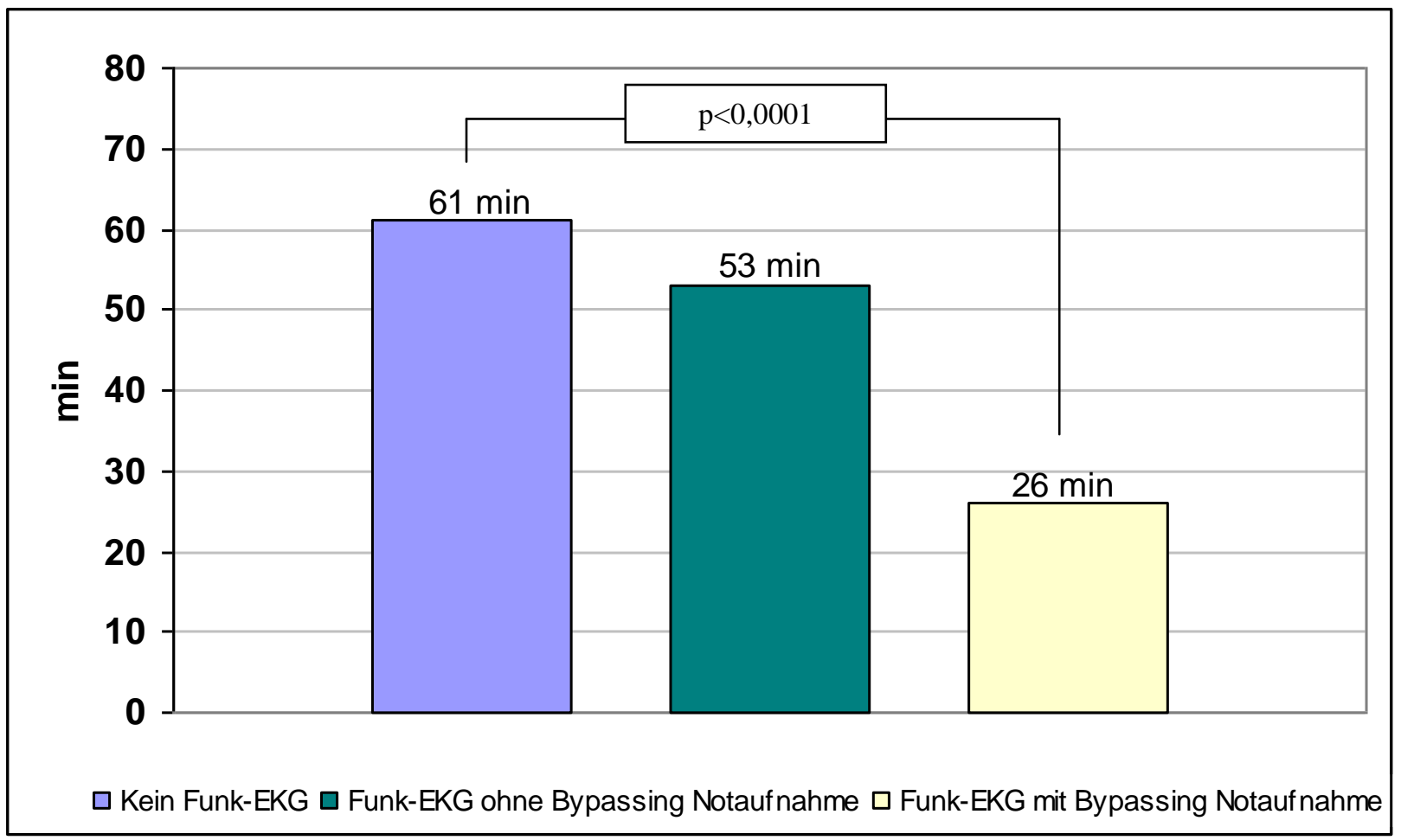

Abbildung 20: door-to-balloon-Zeiten in Abhängigkeit von Funk-EKG und Bypassing der Notaufnahme (Median) 


\subsection{Verstorbene Patienten}

Im Gesamtkollektiv sind 10 Patienten während des Krankenhausaufenthaltes verstorben $(8,8 \%)$. Eine Patientin verstarb am 32. Tag ihrer Hospitalisierung, so dass die 30-TagesMortalität 7,9\% betrug. Nach Ablauf eines halben Jahres war ein weiterer Patient aus dem I. als auch aus dem IV. Quartal verstorben, so dass die 6-Monats-Mortalität bei 10,5\% lag Betroffen waren 3 Patienten im I. Quartal (9,1\%), ein Patient im II. Quartal (3,6\%), 3 Patienten im III. Quartal (12,0 \%) und 3 Patienten im IV. Quartal (10,7\%). Die verstorbenen Patienten waren mit einem mittleren Alter von 76,7 Jahre im Durchschnitt 10 Jahre älter als das Gesamtkollektiv. Sechs der 10 Verstorbenen waren männlich. Insgesamt 8 der 10 Patienten waren direkt mit dem Notarzt in die Interventionsklinik transportiert worden, die 2 übrigen hatten sich in einem Fall selbstständig in der Notaufnahme der Interventionsklinik vorgestellt bzw. im anderen Fall als Sekundärtransport die Interventionsklinik erreicht. Vergleichbar mit dem Gesamtkollektiv betrug die mittlere door-to-balloon-Zeit bei den Verstorbenen $47 \mathrm{~min}$ (Median: $31 \mathrm{~min}$ ), die contact-to-balloon-Zeit lag im Durchschnitt bei 96 min (Median: $78 \mathrm{~min}$ ). Alle verstorbenen Patienten befanden sich zum Zeitpunkt der Aufnahme in der Interventionsklinik im kardiogenen Schock bzw. unter protrahierter Reanimation. (Tabelle 11).

\begin{tabular}{|c|c|c|c|c|c|c|c|c|c|c|}
\hline \multirow[b]{2}{*}{ No. } & \multicolumn{3}{|c|}{$\begin{array}{c}\text { I. } \\
\text { Quartal }\end{array}$} & \multirow{2}{*}{$\begin{array}{c}\begin{array}{c}\text { II. } \\
\text { Quartal }\end{array} \\
\text { Pat. } 4 \\
\end{array}$} & \multicolumn{3}{|c|}{$\begin{array}{c}\text { III. } \\
\text { Quartal }\end{array}$} & \multicolumn{3}{|c|}{$\begin{array}{c}\text { IV. } \\
\text { Quartal }\end{array}$} \\
\hline & Pat.1 & Pat.2 & Pat.3 & & Pat. 5 & Pat. 6 & Pat 7 & Pat. 8 & Pat. 9 & Pat.10 \\
\hline Alter (Jahre) & 84 & 67 & 69 & 81 & 53 & 96 & 76 & 83 & 79 & 79 \\
\hline $\begin{array}{c}\text { Geschlecht } \\
\text { (M/W) }\end{array}$ & W & M & M & $\mathrm{M}$ & M & W & $M$ & W & W & M \\
\hline $\begin{array}{c}\text { Art der } \\
\text { Einweisung }\end{array}$ & SV & PT & PT & PT & PT & PT & ST & PT & PT & PT \\
\hline $\begin{array}{l}\text { "door-to- } \\
\text { balloon" } \\
\text { (min) }\end{array}$ & 74 & 45 & 117 & 93 & 35 & 21 & 16 & 23 & 20 & 26 \\
\hline $\begin{array}{c}\text { "contact-to- } \\
\text { balloon" } \\
(\mathrm{min})\end{array}$ & 74 & 82 & 167 & 137 & 122 & 66 & 134 & 67 & 50 & 61 \\
\hline $\begin{array}{l}\text { Hospitalisie- } \\
\text { rungszeit } \\
\text { (d) }\end{array}$ & 3 & 4 & 7 & 1 & 9 & 2 & 10 & 32 & 1 & 1 \\
\hline $\begin{array}{l}\text { Todes- } \\
\text { Ursache }\end{array}$ & $\begin{array}{c}\text { Kardiogener } \\
\text { Schock, } \\
\text { Reanimation } \\
\text { bei } \\
\text { Asystolie, } \\
\text { Haupt. } \\
\text { staum- } \\
\text { stenose }\end{array}$ & $\begin{array}{l}\text { Kardiogener } \\
\text { Schock, } \\
\text { distale } \\
\text { Haupt- } \\
\text { stamm- } \\
\text { stenose, } \\
\text { IABP }\end{array}$ & $\begin{array}{c}\text { VU bei KF } \\
\text { (CT vor } \\
\text { HK), } \\
\text { kardio- } \\
\text { gener } \\
\text { Schock, } \\
\text { IABP, } \\
\text { hypox.. } \\
\text { Hirnscha- } \\
\text { den }\end{array}$ & 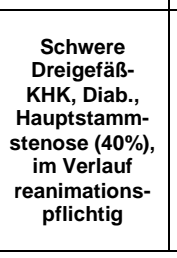 & \begin{tabular}{|c} 
Protr. \\
Reanima- \\
tion am \\
Einsatzort, \\
yypox. \\
Hirnscha- \\
den
\end{tabular} & $\begin{array}{l}\text { Kardio- } \\
\text { gener } \\
\text { Schock }\end{array}$ & $\begin{array}{l}\text { Kardiog. } \\
\text { Schock, } \\
\text { Haupt- } \\
\text { stamm- } \\
\text { stenose, } \\
\text { ACVB } \\
\text { geplant, } \\
\text { Multi- } \\
\text { organ- } \\
\text { versagen }\end{array}$ & $\begin{array}{l}\text { Kardiog. } \\
\text { Schock, } \\
\text { rez, } \\
\text { untere } \\
\text { GlB bei } \\
\text { Ulcus } \\
\text { Bauhin- } \\
\text { Klappe }\end{array}$ & $\begin{array}{l}\text { Kardio- } \\
\text { gener } \\
\text { Schock }\end{array}$ & $\begin{array}{c}\text { Kardio- } \\
\text { gener } \\
\text { Schock, } \\
\text { IABP }\end{array}$ \\
\hline
\end{tabular}

Tabelle 11: Verstorbene Patienten (*PT=Primärtransport, $S T=$ Sekundärtransport, $S V=$ Selbsteinweisung) 


\section{Diskussion}

\subsection{Prozessoptimierung durch standardisiertes Qualitätsmanagement}

Die schnellstmögliche Wiedereröffnung des verschlossenen Herzkranzgefässes stellt die vorrangige Behandlungsstrategie zur Reduktion von Mortalität und Morbidität bei Patienten mit akutem ST-Streckenhebungsinfarkt (STEMI) dar. Zahlreiche Studien haben belegt, dass unter optimalen Bedingungen die primäre perkutane Koronarintervention (PCI) der Fibrinolyse-Therapie überlegen ist (Andersen et al. 2003, Keeley et al. 2003, Widimsky et al. 2003). Insbesondere in der frühen Infarktphase (innerhalb der ersten 2-3 Stunden) scheint die Prognose wesentlich von der Zeitdauer von Symptombeginn bis zur Wiedereröffnung des Infarktgefäßes abhängig zu sein (Berger et al. 1999, Cannon et al. 2000). Eine prospektive Kohortenstudie konnte kürzlich unter Einschluss von mehr als 43.000 Patienten aus dem American-College-of-Cardiology-National-Cardiovascular-Datenregister zeigen, dass eine verlängerte door-to-balloon-Zeit mit erhöhter Mortalität assoziiert ist. Dies galt sogar für diejenigen Patienten, die bereits innerhalb der geforderten door-to-balloon-Zeit von $90 \mathrm{~min}$ behandelt wurden (Rathore et al. 2009). Aus diesem Grund wird sowohl national als auch international der wesentliche Focus bei der Infarktbehandlung auf die Reduktion der Reperfusionszeiten gelegt. Sowohl im europäischen Raum als auch in den USA gelingt es jedoch nur einer geringen Anzahl von Krankenhäusern, die geforderten Zielkriterien einzuhalten (Nallamothu et al. 2005, Zahn et al. 2006). Auch ließ sich trotz intensiver Bemühungen über mehrere Jahre keine Verbesserung der Reperfusionszeiten erzielen (McNamara et al. 2006 b).

Kürzlich wurden von Bradley et al. in einer retrospektiven Beobachtungsstudie verschiedene spezifische Strategien identifiziert, die mit einer signifikanten Verkürzung der Reperfusionszeiten assoziiert sind. $\mathrm{Zu}$ diesen Strategien zählen die Alarmierung des Katheterpersonals aufgrund eines prähospital angefertigten EKG, während sich der Patient noch auf dem Weg in das Krankenhaus befindet, die Durchführung der Alarmierung durch den diensthabenden Arzt der Notaufnahme ohne Miteinbeziehung des zuständigen Kardiologen sowie die Aktivierung des Katheterassistenzpersonals und des Interventionskardiologen durch eine zuvor durch einen einzigen Anruf der Notaufnahme kontaktierte zentrale Vermittlungsstelle. 
Weitere identifizierte Strategien waren die Forderung an das Katheterpersonal, innerhalb von 20 min nach Alarmierung im Katheterlabor einzutreffen, die ständige Anwesenheit des diensthabenden Kardiologen vor Ort sowie die unmittelbare Rückkopplung der Ergebnisse an das Personal des Katheterlabors sowie der Notaufnahme (Bradley et al. 2006a).

Das in der vorliegenden Untersuchung beschriebene regionale Herzinfarktnetz in ländlicher Region setzt sich aus einer PCI-Klinik mit 24h-Bereitschaft und mehreren erfahrenen Interventionskardiologen, 2 Nicht-Interventionskliniken sowie dem lokalen RettungsdienstSystem zusammen. Seit der Gründung des Infarktnetzes im Jahr 2002 wurde aus einer zunächst nur engen Zusammenarbeit heraus ein seit 2005 standardisiertes und strukturiertes Behandlungskonzept für STEMI-Patienten entwickelt, welches bereits von Anfang an eine Vielzahl der von Bradley et al. identifizierten Strategien zur Verkürzung der Reperfusionszeiten beinhaltete. Eine Rückkopplung der Behandlungsergebnisse hatte bisher jedoch nur unstrukturiert und sporadisch in Einzelfällen stattgefunden.

Bereits im I. Quartal vor Start der Qualitätsmanagement(QM)-Interventionen befanden sich die Reperfusionszeiten im internationalen Vergleich auf einem hohen Ausgangsniveau. So lag der Median der door-to-balloon-Zeiten im Herzinfarktnetz Hildesheim-Leinebergland im I. Quartal 2006 bei 54 min. In der von Bradley et al. durchgeführten Analyse der 365 führenden US-amerikanischen Kliniken betrug der Mittelwert der Mediane hingegen 100,4 min. Nach dieser Erhebung lag der Median der door-to-balloon-Zeiten überhaupt nur bei drei USKliniken unter 60 min (Bradley et al. 2006 a). Die contact-to-balloon-Zeit befand sich bereits im Ausgangsquartal bei 55\% der im Herzinfarktnetz Hildesheim-Leinebergland behandelten Patienten innerhalb der von der Deutschen Gesellschaft für Kardiologie geforderten 120 min (Hamm et al. 2004).

Die Grundlage für das bereits hohe Ausgangsniveau bildet das etablierte klare und verbindliche Ablaufprotokoll für Diagnosestellung, Transport, Alarmierung und Therapie im Herzinfarktnetz Hildesheim-Leinebergland, welches überhaupt erst ein systematisches Vorgehen bei Patienten mit STEMI ermöglicht (Abbildung 3). National und international wurden spezifische Ablaufprotokolle konzipiert, die in Ballungszentren wie auch in weitläufigen Regionen mit langen Anfahrtswegen zur PCI-Klinik den Zugang zur primären PCI und eine Verkürzung der Reperfusionszeiten ermöglichen sollen. 
Der Herzinfarktverbund Essen mit insgesamt zwölf Krankenhäusern und vier Interventionszentren in einem $210 \mathrm{~km}^{2}$ großen Stadtgebiet mit rund 600000 Einwohnern (Bruder et al. 2007) als auch das Rostocker Drip\&Ship Netzwerk mit dem Universitätsklinikum als singulärem Interventionszentrum und sieben kooperierenden NichtInterventionskliniken in einem Umkreis bis $\mathrm{zu} 65 \mathrm{~km}$ (Schneider et al. 2007) veranschaulichen, dass durch optimierte und effiziente Logistik eine flächendeckende, leitliniengerechte Versorgung von Patienten mit STEMI sowohl vor komplexem Hintergrund einer Großstadt als auch in ländlicher Region mit weiten Transportzeiten gewährleistet werden kann. In zwei kürzlich erschienenen US-amerikanischen Studien konnte gezeigt werden, dass durch Implementierung eines standardisierten Ablaufprotokolls die Behandlung und der Transfer von STEMI-Patienten auch über mehr als 210 Meilen in eine PCI-Klinik unter Berücksichtigung der vorgegebenen einzuhaltenden Reperfusionszeiten sicher und praktikabel ist (Henry et al. 2007, Ting et al. 2007).

In der vorliegenden Arbeit wurde weltweit erstmals der Einfluss einer standardisierten Datenerfassung mit systematischer interaktiver Rückkopplung der Behandlungsergebnisse an alle Beteiligten der Rettungs- und Therapiekette auf die Reperfusionszeiten bei Patienten mit STEMI analysiert. In den Feedback-Prozess wurde dabei explizit und erstmalig auch das präklinische Rettungssystem einbezogen. Es konnte gezeigt werden, dass dieses innovative Konzept mit vergleichsweise simpler QM-Intervention trotz des bereits hohen Ausgangsniveaus zu einer eindrucksvollen Verbesserung der Behandlungszeiten führt. Die intrahospitalen Behandlungszeiten wurden innerhalb eines Jahres praktisch halbiert mit Verkürzung des Medians der door-to-balloon-Zeiten von 54 auf 26 min. Der Median der contact-to-balloon-Zeiten reduzierte sich zeitgleich von 113 auf 74 min. Im Durchschnitt kam es zu einer Verkürzung der Behandlungszeit vom Erstkontakt mit dem Rettungssystem bis zur Wiedereröffnung des verschlossenen Herzkranzgefäßes um 53 min pro Patient. In der retrospektiven Querschnittstudie von Bradley et al. dagegen wurde bei Umsetzung spezifischer Strategien in einer multivariaten Analyse lediglich ein Zeitgewinn von maximal 15 min erreicht (Bradley et al. 2006 a).

Die erzielte Reduktion der contact-to-balloon-Zeit lässt sich sowohl auf die Verkürzung der prä- als auch der intrahospitalen Abläufe innerhalb der Behandlungskette zurückführen. Dabei kommt der Aufenthaltsdauer des Rettungsteams am Einsatzort, der Aufenthaltsdauer des Patienten im Krankenhaus vor Eintreffen im HK-Labor sowie der Dauer der Vorbereitung als auch der Therapie im Herzkatheterlabor ein besonderer Stellenwert zu. 
Bereits vor mehr als 3 Jahrzehnten kamen Qualitätsmanagement(QM)-Maßnahmen zur Optimierung von Prozessabläufen sowohl in der produzierenden Industrie als auch in nichtproduzierenden Bereichen wirkungsvoll zur Anwendung (Nadler 1977). Ende der 90er Jahre wurden erstmals auch im medizinischen Sektor QM-Projekte mit dem Ziel der Umsetzung einer leitliniengerechten pharmakologischen Behandlung bei Infarktpatienten initiiert (Berwick 1989). Nachfolgende Studien konnten zeigen, dass die Optimierung der Pharmakotherapie bei Infarktpatienten mit einer Reduktion der 30-Tages- und 1Jahresmortalität verbunden ist. Als entscheidende QM-Intervention kam dabei eine gezielte Datenrückkopplung zum Einsatz (Marciniak et al. 1998, Soumerai et al. 1998). In einer kürzlich von Brush et al. veröffentlichten Studie wurde die Implementierung eines Programms zur kontinuierlichen Qualitätsverbesserung in der Interventionskardiologie und Kardiochirurgie eines großen kommunalen Krankenhauses vorgestellt. Dies führte zwar nachgewiesenermaßen zu einer Verbesserung der Ergebnisqualität, war jedoch insgesamt mit aufwendigen Prozeduren verbunden (Brush et al. 2006). Insgesamt haben diese Arbeiten aus ganz unterschiedlichen Einsatzfeldern oder -bereichen gezeigt, dass die systematische Datenrückkopplung im Wesentlichen durch die Verbesserung des individuellen Verständnisses für den Gesamtprozess, durch die Erkenntnis der Bedeutung der eigenen Rolle im Gesamtprozess („,buy-in“) sowie durch Etablierung eines Teamgedankens zu einer Verbesserung der Ergebnisqualität führt (Nadler 1977, Brush et al. 2006).

Bradley et al. zeigten 2006 beispielhaft auf, welche Mechanismen der Effizienz von systematischer Datenrückkopplung als QM-Maßnahme in der Herzinfarktversorgung zugrundeliegen. Im Rahmen von detaillierten Interviews wurden die Erfahrungen derjenigen Kliniken aus dem NRMI-Register charakterisiert, denen es zwischen 1999-2002 gelungen war, die door-to-balloon-Zeiten deutlich zu verbessern. Im Vordergrund standen ebenfalls das Engagement und die Motivation, ein definiertes Ziel gemeinsam als Team zu erreichen. Die Einführung von standardisierten und innovativen Ablaufprotokollen mit jedoch flexibler Anpassung und dem Bestreben einer ständigen Optimierung war ein weiterer relevanter Aspekt. In der Zusammenarbeit mit unterschiedlichen Systemen von Abteilungen, Kliniken und Berufsgruppen seien eine sensible Balance und eine gegenseitige Akzeptanz zu bewahren, um Konflikte bei unterschiedlicher Herangehensweise zu umgehen. Hierbei komme der spezifischen Ergebnisrückkopplung, die der frühzeitigen Identifikation von Problemen und der Kommunikation der Fortschritte und erzielten Erfolge dienen soll, eine entscheidende Bedeutung zu. 
Es sei eine Unternehmenskultur anzustreben, die die Ausdauer für neue Herausforderungen, Widerstände und auch Rückschläge in dem Bemühen um Prozessoptimierung steigert (Bradley et al. 2006 b).

Die Akutbehandlung des STEMI stellt nach unseren Erfahrungen einen komplexen und mehrdimensionalen Prozess mit verschiedenen Schnittstellen dar. Eine genaue Kenntnis der Abläufe bei enger Verzahnung der Systeme ist für alle Beteiligte daher obligat: In der Frühphase besteht ein maximaler Zeitdruck, in kurzer Zeit müssen die sichere Diagnosestellung und Therapieeinleitung erfolgen. Verschiedene Systeme müssen alarmiert und informiert werden. Etwa 10-15 verschiedene Personen in jeweils unterschiedlicher Zusammensetzung haben in dieser Phase Direktkontakt zum Patienten. Eine Koordination über unterschiedliche Fachdisziplinen, Abteilung und ggfs. Krankenhäuser mit Kooperation unterschiedlicher Berufsgruppen ist Grundvoraussetzung. Die Einsätze finden rund um die Uhr mit wechselnden Personen statt. Abhängig vom Zeitpunkt der Patientenvorstellung ergeben sich unterschiedliche Problemfelder. Während der regulären Dienstzeit sind möglicherweise der oder die Katheterplätze mit elektiven Patienten oder anderen Notfällen belegt, während im Bereitschaftsdienst das Katheterpersonal bei kurzen Anfahrtsweg des Patienten möglicherweise noch nicht vor Ort ist. Bei den sogenannten Transfer-Patienten aus Nicht-Interventionskliniken gilt es, einen raschen und sicheren Transport zu organisieren.

Letztendlich erwiesen sich die bereits von Bradley et al. genannten Mechanismen zur Effizienz von systematischer Datenrückkopplung in der Herzinfarktversorgung auch in der vorliegenden Arbeit als relevant. Durch die quartalsweisen Feedbackveranstaltungen konnten allen Beteiligten die komplexen Abläufe und die Bedeutung jedes Einzelnen im Gesamtprozess der Akutbehandlung des STEMI nahe gebracht und relevante Probleme erörtert werden. Die Veranstaltungen waren für die Teilnehmer absolut motivationssteigernd und es kam aus dem Team immer wieder zu konstruktiven Verbesserungsvorschlägen. Insbesondere die Innovation, das präklinische Rettungs-System in den Feedback-Prozess mit einzubeziehen, war von entscheidender Bedeutung.

Diese unterschiedlichen Aspekte waren im Zusammenspiel ausschlaggebend für die Verbesserung der Reperfusionszeiten. Aufgrund dieser Ergebnisse müsste das Instrument der standardisierten prospektiven Datenerfassung und systematischen Ergebnisrückkopplung grundsätzlich in die Herzinfarktversorgung einbezogen werden. 


\subsection{Bedeutung von Funk-EKG und Umgehung der Notaufnahme (Bypassing)}

In den Vereinigten Staaten von Amerika präsentieren sich ca. 75\% aller STEMI Patienten primär an Nicht-Interventionskliniken (Garvey et al. 2006). Mehrere randomisierte europäische Studien konnten zeigen, dass der Transfer von STEMI Patienten zur PCI in eine Interventionsklinik einer Lyse-Therapie vor Ort überlegen ist (Andersen et al. 2003, Widimsky et al. 2003). Eine quantitative Auswertung ergab, dass ein solcher Transfer in eine PCI-Klinik bei 1000 behandelten Patienten insgesamt 70 schwere kardiale Ereignisse (Tod, Schlaganfall oder Re-Infarkt) verhindert (Zijlstra 2003). Daten aus dem amerikanischem NRMI-Register offenbaren allerdings, dass der Median der door-to-balloon-Zeiten von Transferpatienten bei 180 min liegt, was bedeutet, dass nur 4\% der Patienten innerhalb der von den Leitlinien geforderten Zeit behandelt werden (Nallamothu et al. 2005). Eine jüngst veröffentlichte Studie beschreibt am Beispiel einer kanadischen Großstadt die Entwicklung eines speziellen Ablaufprotokolls, das einen direkten Transport der STEMI-Patienten in eine PCI-Klinik vorsieht (Bypassing der Nicht-Interventionsklinik). Hierzu wurden zuvor fortgeschrittene Rettungssanitäter (sog. advanced paramedics) in der Erstellung und Interpretation eines 12-Kanal-EKG geschult. Der Median der door-to-balloon-Zeiten der direkt in die Interventionsklinik transportierten Patienten betrug $69 \mathrm{~min}$ im Vergleich zu 123 min bei Transfer-Patienten (Le May et al. 2008). Eine Studie von Bradley et al. ergab, dass die Anfertigung eines 12-Kanal-EKG allein keine signifikante Verbesserung der door-toballoon-Zeit zur Folge hat. Letztendlich war das Ergebnis davon abhängig, ob das EKG an die Interventionsklinik übermittelt wurde und wie diese Information organisatorisch umgesetzt wurde (Bradley et al. 2006a). Die Notwendigkeit und Sinnhaftigkeit einer prähospitalen Triage wird durch die Tatsache unterstrichen, dass in den Vereinigten Staaten fast $80 \%$ der erwachsenen Population nur $60 \mathrm{~min}$ von einer PCI-Klinik entfernt leben. Nahezu 3/4 der Patienten müssten bei Umgehung der Nicht-Interventionsklinik sogar nur weniger als $30 \mathrm{~min}$ Zeitverzögerung in Kauf nehmen. Die mediane Zeitdauer zur nächsten Interventionsklinik beträgt rund 12 min bei einer medianen Distanz von 8 Meilen (Nallamothu et al. 2006).

Direkt erkennbare Vorteile eines prähospital übermittelten Funk-EKG sind die frühzeitige und zuverlässige Diagnose eines STEMI. Das Funk-EKG gewährleistet eine sichere Diagnosestellung in der PCI-Klinik und dient als Entscheidungsgrundlage für die Alarmierung des Katheterteams. 
Darüberhinaus ergibt sich ein Zeitgewinn für die Alarmierung des Katheterpersonals insbesondere bei Patienten mit kurzem Anfahrtsweg, auch muss prä-interventionell in der Klinik kein erneutes EKG mehr geschrieben werden. Einen möglichen Nachteil stellt der geringe Zeitverlust am Einsatzort infolge der 12-Kanal-EKG-Aufzeichnung und Übermittlung dar.

Daten aus dem amerikanischen NRMI-Register ( $\mathrm{n}=56.647$ zwischen 2002 und 2005) offenbaren, dass nur bei ca. jedem 15. STEMI-Patienten ein prähospitales EKG angefertigt worden war. In dieser Gruppe konnte jedoch eine signifikante Verkürzung der Reperfusionszeiten ermittelt werden (Curtis et al. 2006).

Im Herzinfarktnetz Hildesheim-Leinebergland ist in dem Ablaufprotokoll von vorneherein die Übermittlung eines 12-Kanal-EKG an den diensthabenden Arzt der kardiologischen Intensivstation mit absoluter Sach- und Entscheidungskompetenz und paralleler telefonischer Rückkopplung implementiert. Unnötige Alarmierungen des Katheterpersonals werden somit verhindert. Der standardisierte Ablaufprozess trug entscheidend dazu bei, dass sich die doorto-balloon-Zeiten bereits im Ausgangsquartal auf einem hohen Niveau befanden. Auch unterstreichen diese Ergebnisse die Schlüsselstellung des Funk-EKG im Gesamtprozess. Durch die explizite Zielvorgabe des Bypassing der Nicht-Interventionsklinik konnte die Anzahl der Sekundärtransporte im Untersuchungszeitraum reduziert werden. Zuletzt befanden sich unter den Transfer-Patienten nur noch solche, die sich zuvor als Selbstvorsteller in der Nicht-Interventionsklinik vorgestellt hatten bzw. in einem Fall per RTW ohne Möglichkeit der Übermittlung eines 12- Kanal-EKG eingeliefert worden war. Eine signifikante Zeitverzögerung durch Aufzeichnung und Übermittlung des 12-Kanal-EKG konnte nicht nachgewiesen werden.

Kürzlich erhobene Daten des Minneapolis Heart Instituts zeigen auf, dass die door-to-balloonZeiten bei Patienten, die sich entweder direkt und ohne vorherigen Kontakt zum Rettungsdienst bzw. als Primärtransport in der Interventionsklinik vorstellten, $65 \mathrm{~min}$ betrugen, wohingegen die door-to-balloon-Zeiten bei Transfer-Patienten aus einer NichtInterventionsklinik (Ankunft Interventionsklinik bis erste Balloninflation) erheblich kürzer bei etwa 20 min lagen. Bei diesen Transfer-Patienten erlaubte die frühe auf einem prähospital erstelltem Funk-EKG basierende Diagnose eines STEMI die Direktübergabe der Patienten im Katheterlabor unter Umgehung der Notaufnahme (Bypassing der Notaufnahme) (Henry et al. 2007). 
In der vorliegenden Untersuchung lag dementsprechend die Zeitdauer von Ankunft Interventionsklinik bis Ankunft im HK-Labor (door-to-cath) bei Primärtransport-Patienten im Ausgangsquartal bei 19 min (Median). Die zuvor angekündigten Transfer-Patienten, die generell die Notaufnahme umgehen und direkt im HK-Labor an den Interventionskardiologen übergeben werden, erzielten hingegen eine door-to-cath-Zeit von nur 5 min (Median). Dieser Zusammenhang veranschaulicht deutlich, dass im Fall der Vorankündigung von STEMIPatienten durch Übermittlung eines 12-Kanal-EKG mit telefonischer Rückkopplung nicht nur bei Transferpatienten sondern auch im Fall von Primärtransporten eine kurze door-to-cathZeit realisierbar sein sollte.

In der vorliegenden Arbeit lag der Anteil der mit Funk-EKG angekündigten PrimärtransportPatienten mit STEMI bereits im Ausgangsquartal mit 59\% auf einem hohen Niveau. Dieser Anteil stieg im II. Quartal auf 86\% und blieb dann über die nachfolgenden Quartale konstant. Parallel konnte ein kontinuierlicher Anstieg der unter Umgehung der Notaufnahme direkt im HK-Labor an den Interventionskardiologen übergebenen Patienten von initial $23 \%$ im Ausgangsquartal auf 76\% im IV. Quartal dokumentiert werden. Dieser Effekt der besseren Ausnutzung der Funk-EKG mit der Konsequenz der Direktübergabe der Patienten im HKLabor war Folge der systematisch durchgeführten QM-Maßnahmen. Darüber hinaus kann durch die vorliegenden Ergebnisse nachgewiesen werden, dass die prähospitale Registrierung und Übermittlung eines 12-Kanal-EKG $\mathrm{zu}$ einer signifikanten Verbesserung der Reperfusionszeiten bei Primärtransport-Patienten führt. Die contact-to-balloon-Zeit betrug in der Gruppe ohne Funk-EKG im Median 103 min vs. 74 min in der Gruppe mit Funk-EKG. Die mediane door-to-balloon-Zeit verkürzte sich bei Übermittlung eines Funk-EKG von 61 auf 29 min. Der größte Zeitgewinn war in der Gruppe mit Funk-EKG denjenigen Patienten zuzuschreiben, die direkt im HK-Labor übergeben worden waren. In dieser Untergruppe betrug die mediane door-to-balloon-Zeit $26 \mathrm{~min}$ im Gegensatz zu $53 \mathrm{~min}$ bei den Patienten, bei denen trotz Ankündigung mit Funk-EKG keine Direktübergabe erfolgte. Diese Ergebnisse unterstreichen den entscheidenden Beitrag des Bypassing der Notaufnahme nach prähospitaler Diagnosestellung zur Verkürzung der Reperfusionszeiten bei Patienten mit STEMI.

Khot et al. führten durch Etablierung von komplexen Protokollen eine Optimierung der Ablaufprozesse in der Notaufnahme im Umgang mit STEMI-Patienten in einem 591Bettenhaus der Maximalversorgung in den USA herbei. 
Diese Maßnahmen resultierten in einer signifikanten Reduktion der medianen door-toballoon-Zeit von 113,5 auf 75,5 min (Khot et al. 2007). Zweifellos ist die Optimierung der Ablaufprozesse für diejenigen Patienten, die sich ohne vorherigen Kontakt zum Rettungssystem in der Notaufnahme der Interventionsklinik selbst vorstellten, von großer Bedeutung. In der viel größeren Gruppe der Primärtransport-Patienten allerdings erscheint basierend auf den Ergebnissen der vorliegenden Arbeit ein Systemwechsel erforderlich. Bei diesen Patienten muss die Notaufnahme nach prähospitaler EKG-Übermittlung systematisch umgangen und der Patient direkt durch den Notarzt an den Interventionskardiologen im HKLabor übergeben werden.

Zusammenfassend konnte aufgezeigt werden, dass eine systematische Umgehung der Notaufnahme grundsätzlich bei nahezu allen Patienten mit STEMI möglich ist. Auf Basis einer guten Organisation mit verbindlichem Ablaufprotokoll innerhalb eines HerzinfarktNetzes konnte unter gezieltem Einsatz von QM-Maßnahmen der Anteil der Patienten mit Direktübergabe im Herzkatheterlabor quartalsweise kontinuierlich gesteigert werden, wodurch eine signifikante Reduktion der Reperfusionszeiten erzielt wurde.

Der im Herzinfarktnetz Hildesheim-Leinebergland etablierte Prozessablauf mit prähospitaler Übermittlung eines Funk-EKG und Bypassing der Notaufnahme bei Patienten mit STEMI (Scholz et al. 2008) wurde bereits in einem wissenschaftlichen Statement der American Heart Association zur Implementierung und Integration eines prähospitalen EKG in die Behandlung von Akuten Koronarsyndromen ausführlich zitiert (Ting et al. 2008). 


\subsection{Limitationen}

Die vollständige, lückenlose und vor allem valide Erfassung der jeweiligen Einsatzzeiten war mitunter mit erheblichen Schwierigkeiten verbunden. Hier ist insbesondere der Zeitpunkt des Symptombeginns (bzw. Symptommaximum als mutmaßlicher Infarktbeginn) zu nennen, der trotz genauer Nachfrage - von einem Teil der Patienten nur vage angegeben werden kann.

In der vorliegenden Arbeit wurde zwar eine prospektive Datenerfassung mit systematischer und standardisierter Dokumentation und Analyse durchgeführt, allerdings konnte keine Randomisierung der Patienten vorgenommen werden, sondern es erfolgte ein „VorherNachher-Vergleich“. Eine Randomisierung wäre bei dem angewendeten methodischen Konzept kaum logisch durchführbar gewesen. So hätte man bei Randomisierung nur bei einem Teil der jeweiligen Gruppe der Rettungs-und Therapiekette ein Feedback vornehmen dürfen. Aufgrund eines Bias durch ungezielten Informationsaustausch untereinander wären die Vergleichsdaten dann aber kaum valide gewesen.

Es handelt sich um ein umschriebenes Herzinfarktnetz mit begrenzter Patientenanzahl. Trotz niedriger Fallzahlen pro Quartal konnten dennoch hochsignifikante Resultate bezogen auf die Verbesserung der Reperfusionszeiten ermittelt werden. Um jedoch den Effekt einer systematischen Datenrückkopplung bei STEMI-Patienten auf die Letalität zu untersuchen sind große Multicenter-Studien erforderlich.

Das hier angewendete Ablaufprotokoll innerhalb des Herzinfarktnetzes setzt eine gewisse apparative Ausstattung voraus und wurde mit hohem Aufwand etabliert. Auch war das gesamte Projekt nur aufgrund eines großen Engagements aller Beteiligten umsetzbar. Die Fragen sowohl der Nachhaltigkeit der Ergebnisse als auch der Übertragbarkeit des Ansatzes auf andere Infarktversorgungs-Systeme sind daher noch offen und müssen in weiteren Studien untersucht werden. 


\subsection{Ausblick}

Auch im Anschluss an den in der vorliegenden Arbeit beschriebenen Untersuchungszeitraum wurde die systematische Qualitätskontrolle innerhalb des Herzinfarktnetzes HildesheimLeinebergland mit dem Bemühen um eine kontinuierliche Verbesserung fortgeführt. Die Feedbackveranstaltungen finden seit 2007 nur noch $1 \mathrm{x} / \mathrm{Jahr}$ gemeinsam mit dem Rettungsdienst im Rahmen einer Fortbildungsveranstaltung für die Rettungsassistenten statt. Die Prozessabläufe erwiesen sich im klinischen Einsatz als praktikabel und konnten fest etabliert werden. So gelang es, die Behandlungsergebnisse nachhaltig auf hohem Niveau zu stabilisieren (Ahlersmann et al. 2009a). Das QM-Projekt konnte somit in den klinischen Alltag implementiert und zum Standard gemacht werden.

Unter QM-Gesichtspunkten ist der Aspekt der Nachhaltigkeit der erzielten Ergebnisse von entscheidender Bedeutung. In dem Bestreben in einem fließenden System stetig flexibel die standardisierten Abläufe zu optimieren, wurden konstruktive Vorschläge in den Prozess mit aufgenommen. So wurde beispielsweise vereinbart, auch bei noch nicht anwesendem Katheterpersonal während des Rufbereitschaftsdienstes und kurzem Anfahrtsweg der angekündigten STEMI-Patienten dennoch die Notaufnahme zu umgehen und den Patienten direkt im HK-Labor zu übergeben. Dabei nimmt der zuvor als Ansprechpartner fungierende diensthabende Arzt der kardiologischen Intensivstation den Patienten zunächst im Katheterlabor in Empfang, und der Patient wird vom Rettungsdienst direkt auf den Kathetertisch umgelagert. Darüber hinaus wurden die Dienstpläne des Katheterpersonals so gestaltet, dass sich unter den Diensthabenden mindestens einer mit einem kurzen Anfahrtsweg befindet, der im Einsatzfall grundsätzlich auch jeweils als erster telefonisch informiert wird. Durch dieses weiterentwickelte Konzept konnten im speziellen Einzelfall erhebliche Zeitgewinne realisiert werden.

Auch wurden in der Auswertung der Daten neue Aspekte zur exakteren Differenzierung mit aufgenommen. So wurden die Intrahospitalinfarkte gesondert aufgeführt und nicht mehr der Gruppe der Selbstvorsteller zugeordnet. Diejenigen Patienten, die per RTW die Interventionsklinik erreichten, zählten im Verlauf zur Primärtransportgruppe mit einer Differenzierung zwischen NEF und RTW als Transportmedium. In der Gruppe der TransferPatienten aus Nicht-Interventionskliniken wurde die door-to-balloon-Zeit unter Berücksichtigung der door-to-door-Zeit (Ankunft Nicht-Interventionsklinik bis Ankunft Interventionsklinik) um den Begriff der first-door-to-balloon-Zeit erweitert. 
Auf den Erfahrungen im Jahr 2006 aufbauend wurde der Dokumentationsbogen (Abbildung 4) im Folgejahr inhaltlich verbessert und in Zusammenarbeit mit der Fa. Dokuform professionell gestaltet (s. Anhang 7.4.). Im Herbst 2007 gelang ein weiterer deutlicher Fortschritt dadurch, dass der bisher sehr aufwendige Prozess der Datenerfassung und -analyse anhand des neuen Dokumentationsbogens nun web-basiert erfolgen konnte. Die erfassten Daten werden auf diese Weise digitalisiert und computergestützt ausgewertet. Diese Entwicklung stellte einen entscheidenden Schritt in dem Bemühen um Praktikabilität und Automatisierung dar. Erst mit Hilfe dieser modernen Informationstechnologie erschien die Option einer eventuellen Übertragbarkeit auf andere Kliniken realisierbar.

Daten aus dem US-amerikanischen NRMI-Register mit mehr als 29.000 STEMI-Patienten belegen, dass sich bei door-to-balloon-Zeiten zwischen 90 und 150 Minuten die Krankenhaussterblichkeit pro 15-min-Zeitverlust um 6,3 Todesfälle je 1000 behandelte Patienten erhöht (McNamara 2006a). In der vorliegenden Arbeit war die Patientenanzahl zu gering, um eine statistisch signifikante Aussage über eine eventuelle Letalitätssenkung durch aktive Zeitgewinne auf dem Boden von systematischen QM-Maßnahmen in der Behandlung von STEMI-Patienten zu treffen.

Aus diesem Ansatz heraus wurde das FITT-STEMI-Projekt (Feedback Intervention and Treatment Times in ST-Elevation Myocardial Infarction) entwickelt (http://www.clinical.trials.gov). Hierfür fungierte die vorliegende Arbeit als Pilotstudie. In der FITT-STEMI-Probephase wurde die Übertragbarkeit des hier vorgestellten Konzepts auf andere Kliniken sowie die generelle Umsetzbarkeit mit einheitlicher Web-Datenerfassung, zentraler Datenanalyse, zentral gesteuerter einheitlicher Ergebnisrückkopplung und Durchführung eines unabhängigen Monitoring an 6 unterschiedlichen PCI-Klinik-Systemen überprüft. Diese multizentrische Studie sollte zeigen, ob durch standardisierte Datenerfassung mit systematischer Rückkopplung der Behandlungsergebnisse die Ergebnisqualität bei STEMI-Patienten verbessert werden kann. Erste Ergebnisse weisen darauf hin, dass das Konzept eines systematischen Daten-Feedbacks unabhängig von der bestehenden Organisationsstruktur auch an unterschiedlichen Kliniken gut praktikabel ist. Bereits nach dem 1. Feedback konnte eine deutliche Verkürzung der Reperfusionszeiten ermittelt werden. (Ahlersmann et al. 2009b). FITT-STEMI soll im weiteren Studienverlauf zeigen, ob und bei welchen Patienten insbesondere unter Berücksichtigung einer Risikostratifizierung mit dem TIMI-Risk-Score durch die Zeitgewinne auch eine Verbesserung der Prognose erzielt werden kann (Maier SK et al. 2009). 


\section{Zusammenfassung}

Bei Patienten mit akutem ST-Streckenhebungsinfarkt stellt die Reperfusion des verschlossenen Herzkranzgefäßes das vorrangige Behandlungsziel dar. Zahlreiche Untersuchungen ergaben Hinweise darauf, dass verlängerte Reperfusionszeiten wahrscheinlich mit einer erhöhten Sterblichkeit einhergehen. In den nationalen und internationalen Leitlinien wurde daher der Fokus auf die Reduktion der door-to-balloon- und contact-to-balloon-Zeiten gelegt. In den zurückliegenden Jahren wurden weltweit Anstrengungen unternommen, die Behandlungszeiten zu verbessern. Dennoch gelingt es nach wie vor in vielen Fällen nicht, die hohen Anforderungen der Leitlinien einzuhalten.

In der vorliegenden prospektiv durchgeführten Untersuchung wurde am Beispiel eines bereits seit Jahren etablierten regionalen Herzinfarktnetzes mit optimaler Basisstruktur und festem Ablaufprotokoll in der Diagnose- und Therapiekette (Übermittlung eines 12-Kanal-EKG mit Bypassing der Nicht-Interventionsklinik und Bypassing der Notaufnahme der Interventionsklinik) der Frage nachgegangen, wie hoch der Anteil der Patienten ist, bei denen die Anforderungen der Leitlinien unter ,real-life“-Bedingungen tatsächlich erfüllt werden können. Darüber hinaus wurde der Einfluss von standardisiertem Qualitätsmanagement mit systematischer Ergebnisrückkopplung auf die Behandlungszeiten analysiert. Innerhalb eines definierten 12-monatigen Untersuchungszeitraums wurden die zeitlichen Abläufe in der Diagnose- und Therapiekette bei allen Patienten mit STEMI und durchgeführter primärer PCI zeitlich exakt und prospektiv erfasst. Die Ergebnisse wurden quartalsweise systematisch allen an der Behandlungs- und Therapiekette beteiligten Berufsgruppen in gemeinsamen interaktiven Veranstaltungen präsentiert.

Im Verlauf der Untersuchung verkürzte sich die door-to-balloon-Zeit im Median von 54 min im I. Quartal auf $26 \mathrm{~min}$ im IV. Quartal. Die contact-to-balloon-Zeit betrug im Ausgangsquartal im Median 113 min und konnte über die nachfolgenden Quartale auf 74 min im IV. Quartal reduziert werden. Der Anteil der Patienten, deren contact-to-balloon-Zeit unter 90 min lag, stieg hochsignifikant von $21 \%$ im I. Quartal auf $79 \%$ im IV. Quartal. In der Gruppe der Primärtransporte kam es zu einem deutlichen Anstieg des Anteils der Patienten, die unter Umgehung der Notaufnahme direkt vom Notarzt an den Interventionskardiologen übergeben werden konnten (von 23\% im I. Quartal auf 76\% im IV. Quartal). 
Die door-to-balloon-Zeiten unterschieden sich in der Primärtransportgruppe bei Patienten ohne Funk-EKG mit 61 min im Median nicht signifikant von der Gruppe der Primärtransporte mit Funk-EKG, wenn keine Direktübergabe im HK-Labor erfolgte (Median: 53 min). Dagegen war in der Gruppe der Primärtransport-Patienten mit Funk-EKG, die unter Umgehung der Notaufnahme direkt vom Notarzt an den Interventionskardiologen im HKLabor übergeben wurden, die door-to-balloon-Zeit signifikant reduziert (Median: $26 \mathrm{~min}$ ).

Die vorliegende Untersuchung zeigt, dass trotz eines bereits hohen Ausgangsniveaus durch die vergleichsweise simple Intervention einer standardisierten Datenerfassung und systematischen Rückkopplung der Behandlungsergebnisse an alle Beteiligten der Rettungsund Therapiekette ein zusätzlicher signifikanter Zeitgewinn erreicht werden konnte. Bereits kurzfristig nach 3 Monaten und kontinuierlich über den gesamten 12-monatigen Studienzeitraum kam es zu einer signifikanten Verbesserung der Behandlungszeiten. Auf der Basis einer guten Organisation mit verbindlichem Ablaufprotokoll konnte unter gezieltem Einsatz von QM-Maßnahmen der Anteil der Patienten mit Direktübergabe im HK-Labor quartalsweise kontinuierlich gesteigert werden, was einen entscheidenden Beitrag zur Reduktion der Reperfusionszeiten leistete.

Die vorliegende Arbeit stellt weltweit die erste prospektiv durchgeführte Untersuchung dar, die den alleinigen Effekt einer systematischen Rückkopplung der Behandlungsergebnisse auf die Reperfusionszeiten bei Patienten mit STEMI analysiert. Darüberhinaus wurde erstmals auch das Rettungssystem konsequent in das Gesamtkonzept einbezogen. Hierdurch kam es zu einer deutlichen Verbesserung der Prozessqualität mit signifikanter Verkürzung der Reperfusionszeiten.

Um jedoch den möglichen Effekt einer Ergebnisrückkopplung auf die klinischen Resultate v.a. die Letalität - bei STEMI-Patienten zu untersuchen (Ergebnisqualität), sind großangelegte Multicenter-Studien erforderlich. Aus diesem Ansatz heraus wurde das FITT-STEMI-Projekt (Feedback Intervention and Treatment Times in ST-Elevation Myocardial Infarction) entwickelt. Die vorliegende Untersuchung fungierte hierfür als Pilotstudie. In der Probephase von FITT-STEMI wurde im Sinne einer Machbarkeitsstudie die Übertragbarkeit des hier vorgestellten Feedback-Konzepts auf andere Kliniken sowie die generelle Umsetzbarkeit und Nachhaltigkeit der Ergebnisse mit einheitlicher Web-Datenerfassung, zentraler Datenanalyse, zentral gesteuerter einheitlicher Ergebnisrückkopplung und Durchführung eines unabhängigen Monitoring an unterschiedlichen PCI-Klinik-Systemen überprüft. 
Erste Ergebnisse weisen darauf hin, dass das Konzept eines systematischen Daten-Feedbacks unabhängig von der bestehenden Organisationsstruktur auch an unterschiedlichen Kliniken gut praktikabel ist. FITT-STEMI soll im weiteren Studienverlauf zeigen, ob und bei welchen Patienten - insbesondere unter Berücksichtigung einer Risikostratifizierung mit dem TIMIRisk-Score - Zeitgewinne tatsächlich zu einer Verbesserung der Prognose führen. 


\section{Anhang}

\subsection{Abbildungsverzeichnis}

Abbildung 1: Landkreis Hildesheim mit geografischer Lage der am Infarktnetz Hildesheim-Leinebergland beteiligten Kliniken

Abbildung 2: Algorithmus-Ablaufprotokoll STEMI-Behandlung im Herzinfarktnetz Hildesheim-Leinebergland 2005

Abbildung 3: 12-Kanal-EKG ,corpuls 08/16“

Abbildung 4: Dokumentationsbogen Infarktnetz Hildesheim-Leinebergland 2006

Abbildung 5: Anzahl der eingeschlossenen Patienten im Quartalsvergleich, unterteilt nach Art der Einweisung

Abbildung 6: Herkunft Patienten I. Quartal

Abbildung 7: Herkunft Patienten II. Quartal

Abbildung 8: Herkunft Patienten III. Quartal

Abbildung 9: Herkunft Patienten IV. Quartal

Abbildung 10:Zeitintervalle der Gesamtgruppe im Quartalsvergleich (Mittelwert)

Abbildung 11: Kaplan-Meier-Kurve: contact-to-balloon-Zeit Gesamtgruppe im Quartalsvergleich

Abbildung 12: Gesamtgruppe: Anteil der Patienten mit contact-to-balloon-Zeit $<90 \mathrm{~min}$

Abbildung 13: Kaplan-Meier-Kurve: door-to-balloon-Zeit Gesamtgruppe im Quartalsvergleich

Abbildung 14: Kaplan-Meier-Kurve: door-to-cath-Zeit Gesamtgruppe im Quartalsvergleich

Abbildung 15:Zeitintervalle Gruppe der Primärtransporte im Quartalsvergleich (Mittelwert)

Abbildung 16: Patienten mit Primärtransport: Anteil der Patienten mit einer contact-to-balloon-Zeit $<90 \mathrm{~min}$

Abbildung 17: Zeitintervalle Gruppe der Patienten mit Sekundärtransport im 
Abbildung 18: Patienten mit Primärtransport: Zeitintervalle der Rettungsund Therapiekette mit und ohne Funk-EKG (Mittelwert)

Abbildung 19: Anteil der Patienten mit Funk-EKG und Bypassing Notaufnahme

Abbildung 20: door-to-balloon-Zeiten in Abhängigkeit von Funk-EKG und Bypassing der Notaufnahme

\subsection{Tabellenverzeichnis}

Tabelle 1: $\quad$ Art der Einweisung

Tabelle 2: $\quad$ Erfasste Zeitpunkte im Dokumentationsbogen

Tabelle 3: Zeitintervalle der Rettungs-und Therapiekette

Tabelle 4: Prähospitalzeit und Revaskularisationszeiten (door-to-balloon und contact-to-balloon)

Tabelle 5: $\quad$ Definition Basischarakteristika

Tabelle 6: Nicht in die Auswertung eingeschlossene Patienten

Tabelle 7: Auswertung Basischarakteristika

Tabelle 8: Zeitintervalle der Rettungs- und Therapiekette in der Gesamtgruppe im Quartalsvergleich (min)

Tabelle 9: Zeitintervalle der Rettungs- und Therapiekette in der Gruppe der Primärtransporte im Quartalsvergleich (min)

Tabelle 10: Zeitintervalle der Rettungs- und Therapiekette in der Gruppe der Sekundärtransporte im Quartalsvergleich (min)

Tabelle 11: Verstorbene Patienten 


\subsection{Abkürzungsverzeichnis}

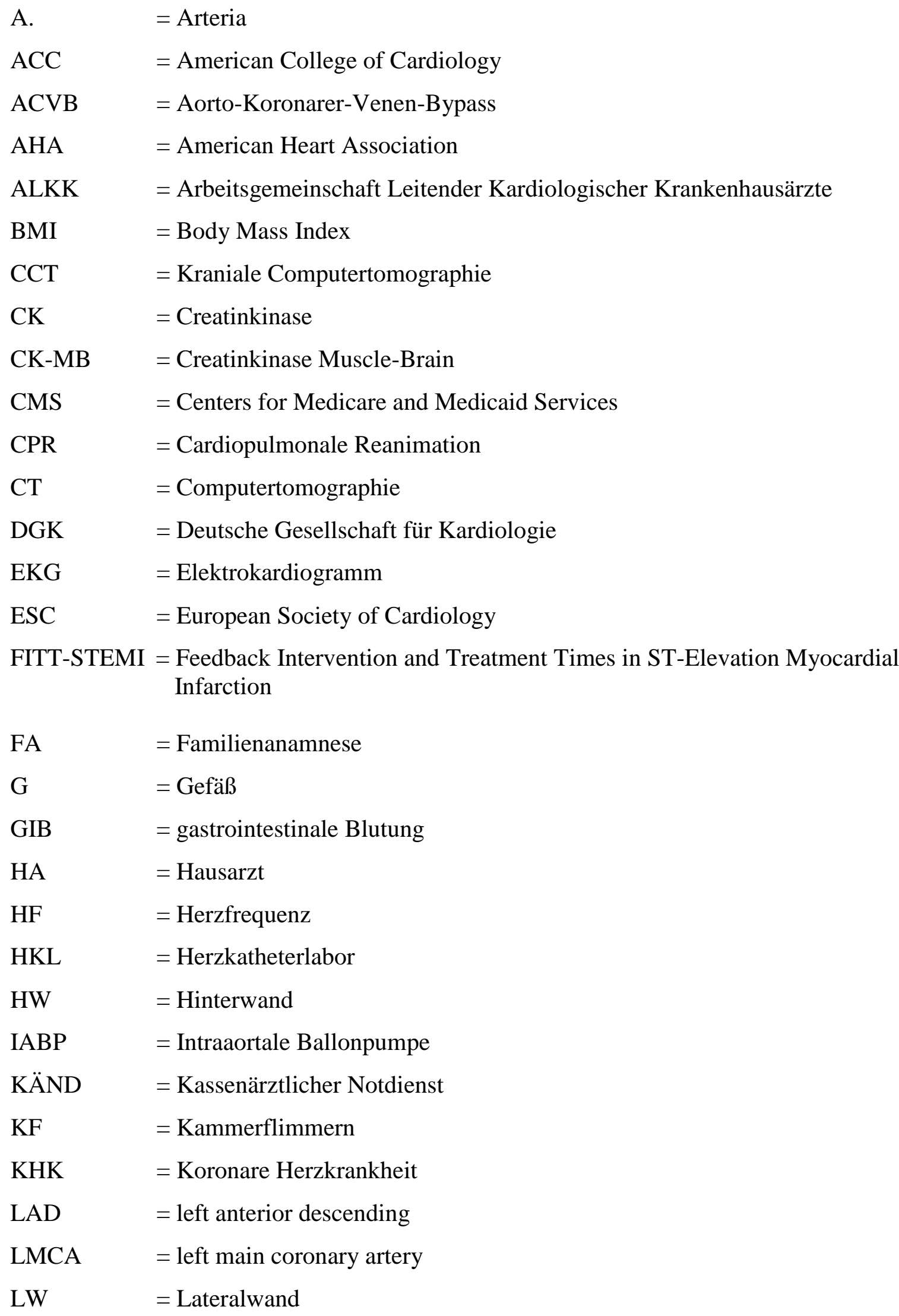




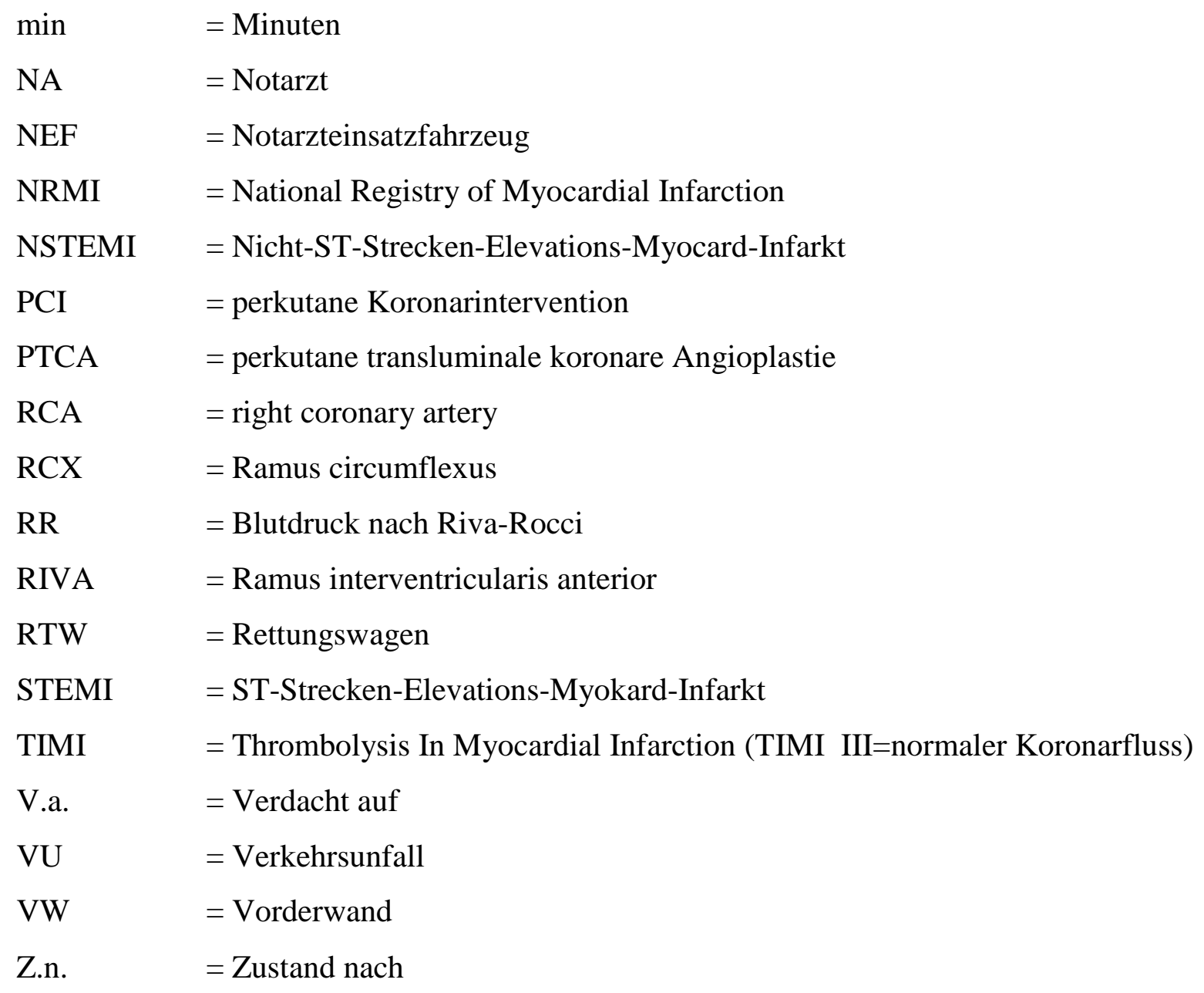




\subsection{Dokumentationsbögen}

\subsubsection{Web-basierter Dokumentationsbogen 10/07}

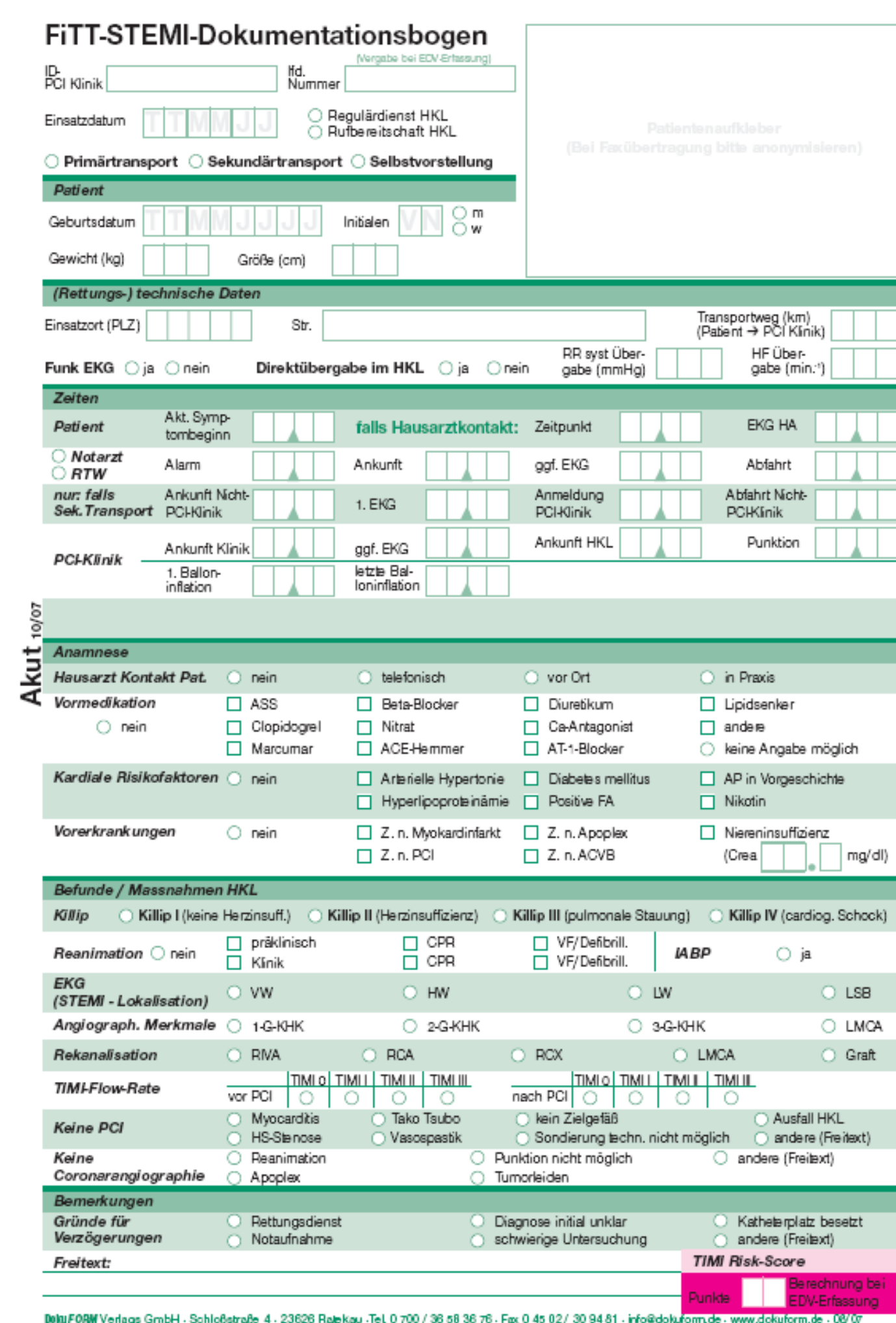




\section{FiTT-STEMI-Dokumentationsbogen}

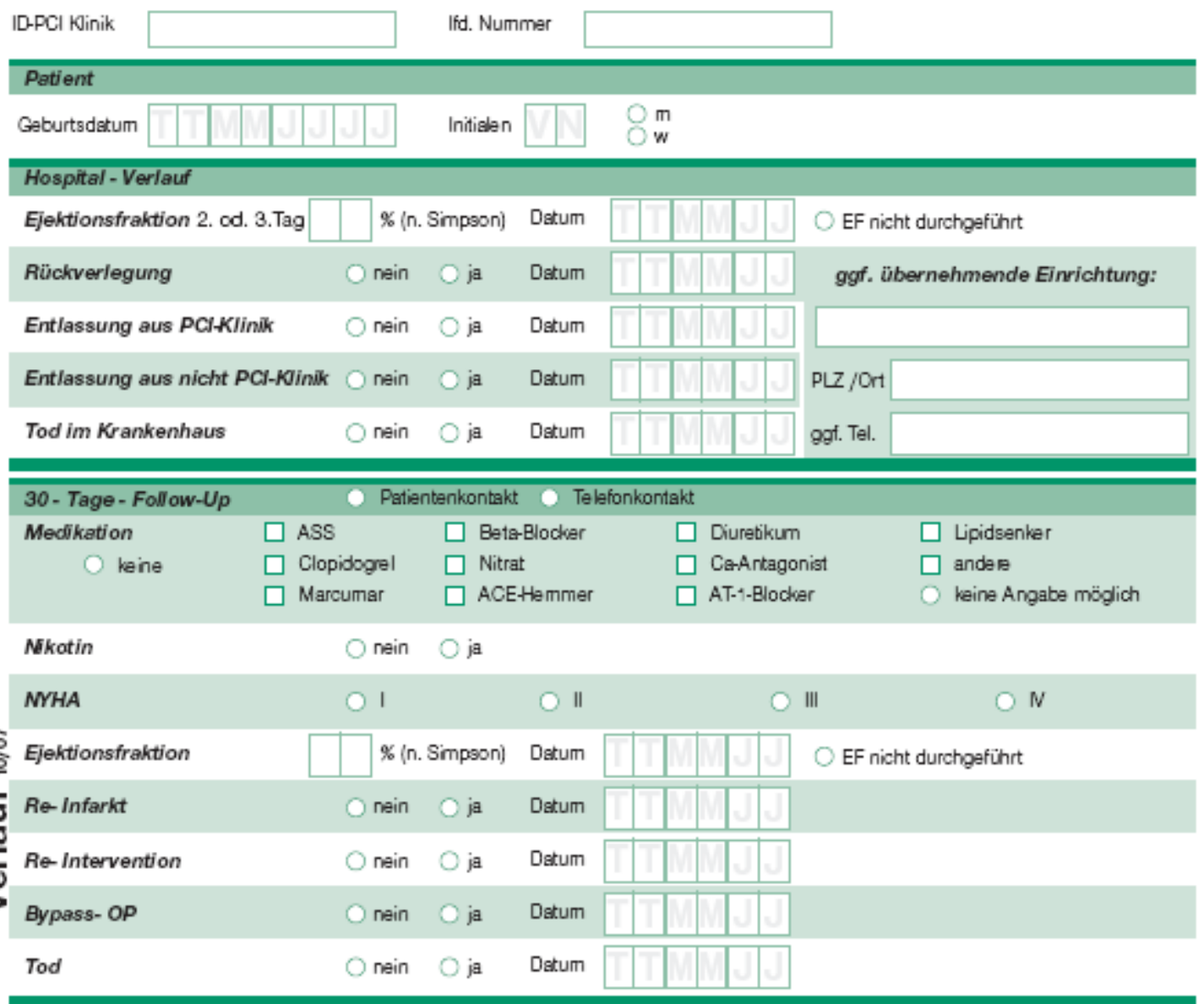

\begin{tabular}{|c|c|c|c|c|}
\hline \multicolumn{5}{|c|}{ 1- Jahr - Follow Up } \\
\hline Mecikation & $\square$ ASS & $\square$ Beta-Blocker & $\square$ Diuretikum & $\square$ Lipidsenker \\
\hline \multirow[t]{2}{*}{ O keine } & $\square$ Clopidogrel & $\square$ Nitrat & $\square$ Ca-Artagonist & $\square$ ander \\
\hline & $\square$ Marcumar & $\square$ ACE-Hemmer & $\square$ AT-1-Blocker & (1) keine Angabe möglich \\
\hline
\end{tabular}

\begin{tabular}{|c|c|c|c|c|c|c|c|}
\hline Nkotin & nain & O ja & & & & & \\
\hline MYHA & 01 & & O $॥$ & & & $\mathrm{O}$ & $O N$ \\
\hline Ejektionsfraktion & $\%<n$. & Simpson) & Datum & $T \pi$ & $\sqrt{3}$ & J & $\mathrm{EF}$ nicht durchgeführt \\
\hline Re-Infarkt & nain & O ja & Detum & $\Gamma$ & $v$ & 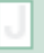 & \\
\hline Re-Intervention & $O$ nain & $O_{j a}$ & Detum & $T$ & 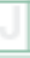 & 0 & \\
\hline Bypass- OP & nein & ○ ja & Datum & $\Gamma$ & 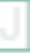 & 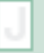 & \\
\hline Tod & nain & $\bigcirc \mathrm{ja}$ & Datum & 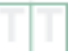 & $\sqrt{3}$ & 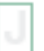 & \\
\hline
\end{tabular}

Petienteneinwilligung

Eirwerstảndniserklärung für 30 Tage - und 1 Jahr Follow-Up-Kontaktaufnahme liegt wor.

ja Onain

Eirwerstảndnis für Oberprüfung der Datenqualität durch einen der Schweigepflicht unterliegenden Mitarbeiter: $\mathrm{O}$ ja Onein 


\subsubsection{Weiterentwickelter Web-basierter Dokumentationsbogen 06/09}

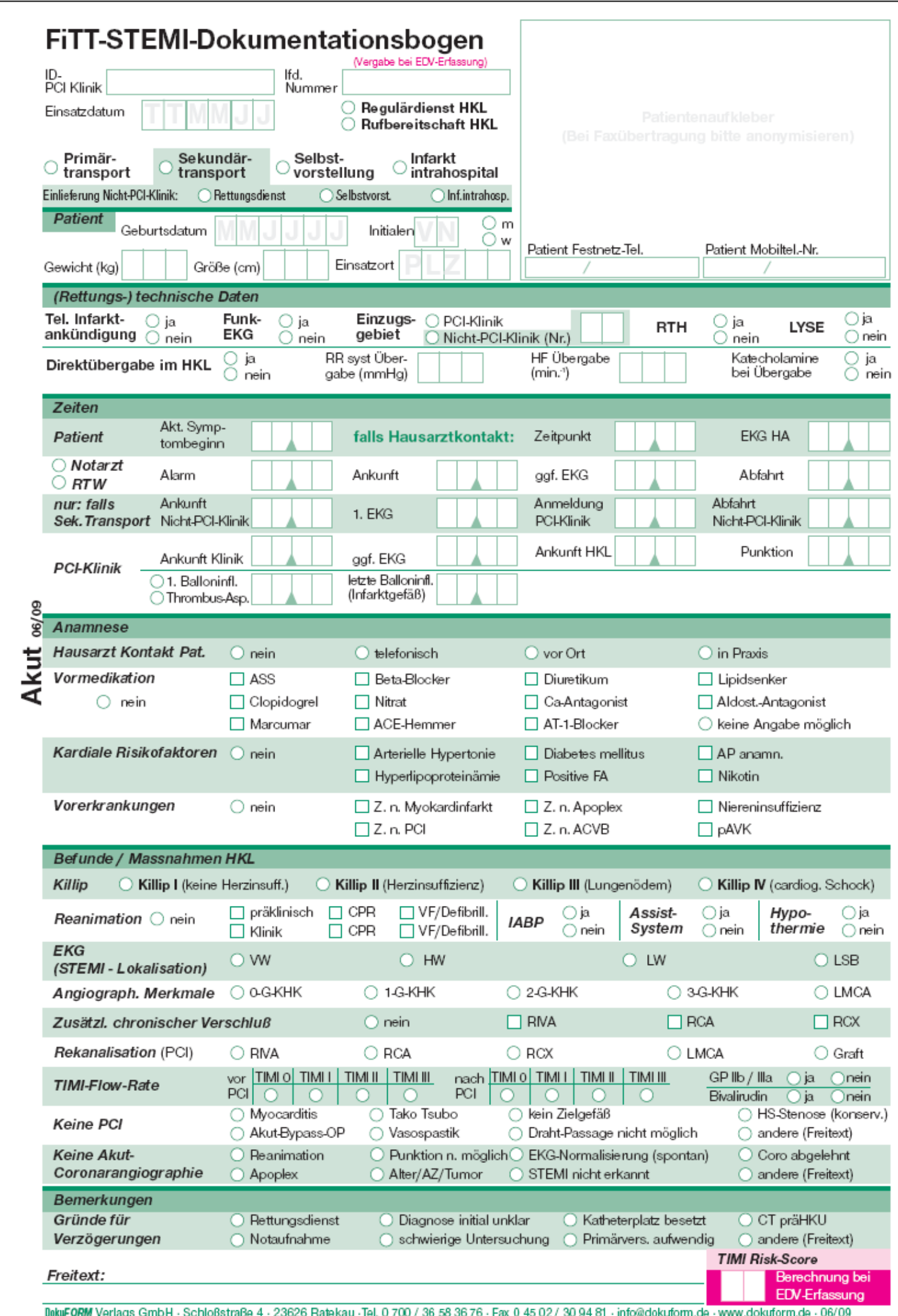




\section{FiTT-STEMI-Dokumentationsbogen (Verlauf)}

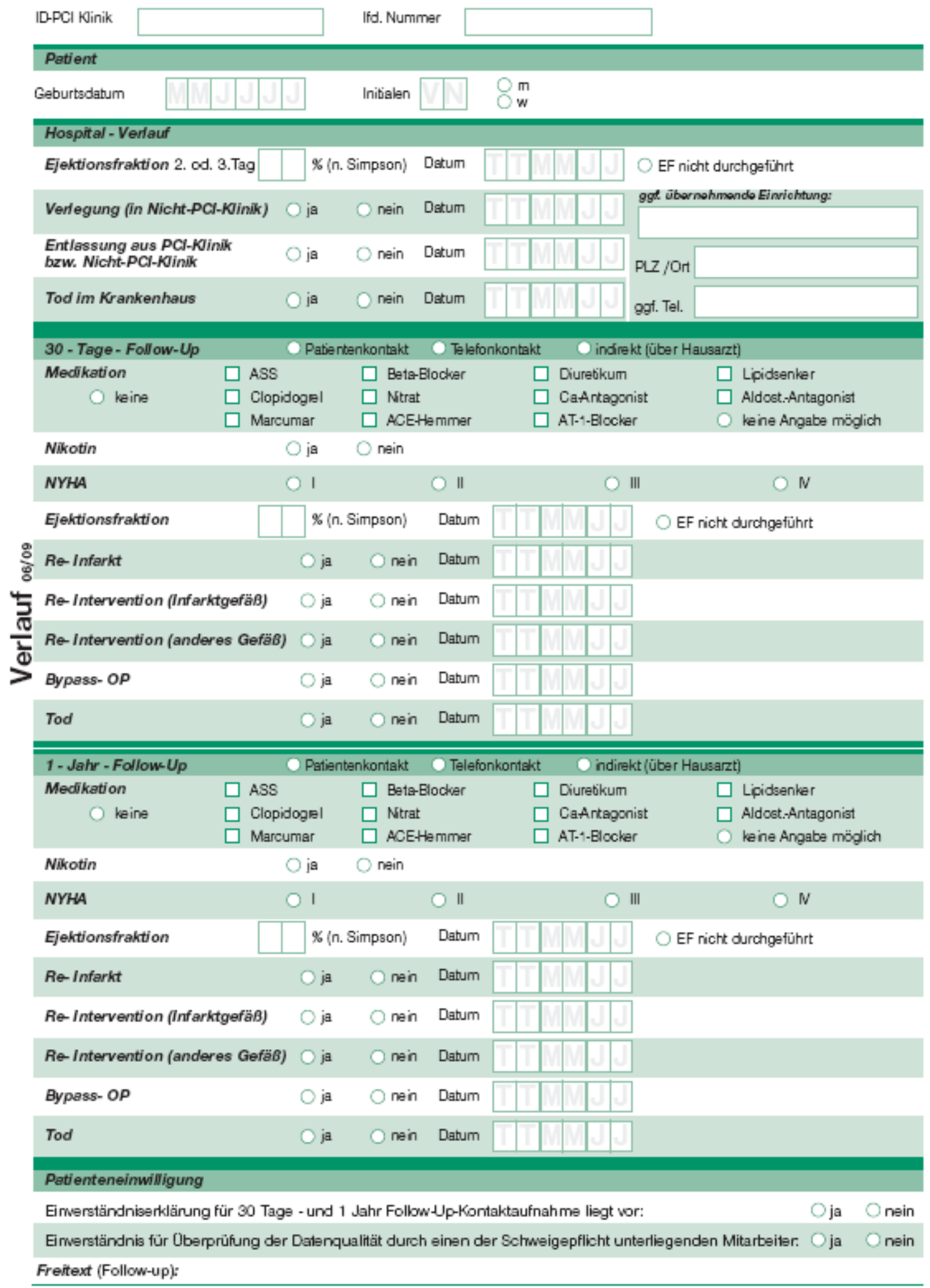




\section{Literaturverzeichnis}

Ahlersmann D, Möller K, Duwald H, Nitsche R, Hilgers R, von Knobelsdorff G, Keating FK, Scholz KH(2009a): Deutliche Verkürzung der Reperfusionszeiten durch systematische Umgehung der Notaufnahme bei Patienten mit akutem ST-Streckenhebungsinfarkt.

Clin Res Cardiol 98, V1601

Ahlersmann D, Fleischmann C, Hilgers R, Jung J, Maier LS, Maier SKG, Olbrich HG, Scholz KH, Werner GS (2009b) Systematische Ergebnisrückkopplung in der Herzinfarktversorgung - Konzeption und Umsetzung der multizentrischen FITT-STEMI-Studie. Clin Res Cardiol $\underline{98}$, P163

Andersen HR, Nielsen TT, Rasmussen K, Thuesen L, Kelbaek H, Thayssen P, Abildgaard U, Pedersen F, Madsen JK, Grande P; DANAMI-2 Investigators (2003): A comparison of coronary angioplasty with fibrinolytic in acute myocardial infarction. New Engl J Med $\underline{349}$, 733-42

Antman EM, Anbe DT, Armstrong PW, Bates ER, Green LA, Hand M, Hochman JS, Krumholz HM, Kushner FG, Lamas GA; American College of Cardiology; American Heart Association Task Force on Practice Guidelines; Canadian Cardiovascular Society (2004): ACC/AHA guidelines for the management of patients with ST-elevation myocardial infarction: a report of the American College of Cardiology/American Heart Association Task Force on Practice Guidelines (Committee to Revise the 1999 Guidelines for the Management of Patients with Acute Myocardial Infarction). Circulation 110, e82-292

Antman EM, Hand M, Armstrong PW, Bates ER, Green LA, Halasyamani LK, Hochman JS, Krumholz HM, Lamas GA, Mullany CJ (2008): 2007 focused update of the ACC/AHA 2004 guidelines for the management of patients with ST-elevation myocardial infarction: a report of the American College of Cardiology/American Heart Association Task Force on Practice Guidelines. J Am Coll Cardiol 51, 210-47 
Berger PB, Ellis SG, Homes DR Jr., Granger CB, Criger DA, Betriu A, Topol EF, Califf RM (1999): Relationship between delay in performing direct coronary angioplasty and early clinical outcome in patients with acute myocardial infarction: results from the Global Use of Strategies to Open Occluded Arteries in Acute Coronary Syndromes (GUSTO-IIb) trial. Circulation $\underline{100}, 14-20$

Berwick DM (1989): Continuous improvement as an ideal in health care. N Engl J Med $\underline{320}$, $53-6$

Bonnefoy E, Lapostolle F, Leizorovicz A, Steg G, Mc Fadden EP, Dubien PY, Cattan S, Boullenger E, Machecourt J (2002): Primary angioplasty versus prehospital fibrinolysis in acute myocardial infarction: a randomised study. Comparison of Angioplasty and Prehospital Thrombolysis in Acute Myocardial Infarction (CAPTIM) study group. Lancet $\underline{360}, 825-829$

Bradley EH, Herrin J, Wang Y, Barton BA, Webster TR, Mattera JA, Roumanis SA, Curtis JP, Nallamothu BK, Magid DJ (2006a): Strategies for reducing the door-to-balloon time in acute myocardial infarction. N Engl J Med 355:2308-20

Bradley EH, Curry LA, Webster TR, Mattera JA, Roumanis SA, Radford MJ, McNamara RL, Barton BA, Berg DN, Krumholz HM (2006b): Achieving rapid door to balloon times: how top hospitals improve complexe clinical systems. Circulation $\underline{113}, 1079-85$

Bruder O, Naber CK, Grosch B, Koslowski B, Benesch L, Budde T, Hailer B, Jacksch R, Erbel R, Sabin G; Herzinfarktverbund Essen (2007): Neue Versorgungsmodelle in der Kardiologie-Herzinfarktverbund Essen. Herz $\underline{32}$, 630-4

Brush JE, Balakrishnan SA, Brough J, Hartman C, Hines G, Liverman DP, Parker JP, Rich J, Tindall N (2006): Implementation of a continuous quality improvement program for percutaneous coronary intervention and cardiac surgery at a large community hospital. Am Heart J $\underline{152}, 379-85$ 
Cannon PB, Gibson CM, Lambrew CT, Shoultz DA, Levy D, French WJ, Gore JM, Weaver WD, Rogers WJ, Tiefenbrunn AJ (2000): Relationship of symptom-onset-to-balloon time and door-to-balloon time with mortality in patients undergoing angioplasty for acute myocardial infarction. JAMA $\underline{283}, 2941-7$

Canto JG, Every NR, Magid DJ, Rogers WJ, Malmgren JA, Frederick PD, French WJ, Tiefenbrunn AJ, Misra VK, Kiefe CI (2000): The volume of primary angioplasty procedures and survival after acute myocardial infarction. National Registry of Myocardial Infarction 2 Investigators. N Engl J Med $\underline{342}$, 1573-80

Centers for Medicare \& and Medicaid Services and the Joint Commission on Accreditation of Healthcare Organization. Specifications Manual for National Hospital Quality Measures, version 2.5 b (2008). Erhältlich unter:

http://jointcommission.org/performancemeasurement/performancemeasurement/current+nhq m+manual.htm

Curtis JP, Portnay EL, Wang Y, McNamara RL, Herrin J, Bradley EH, Magid DJ, Blaney ME, Canto JG, Krumholz HM; National Registry of Myocardial Infarction-4 (2006): The prehospital electrocardiogram and time to reperfusion in patients with acute myocardial infarction, 2000-2002: findings from the National Registry of Myocardial Infarction-4. J Am Coll Cardiol $\underline{47}, 1544-52$

Dalby M, Bouzamondo A, Lechat P, Montalescot G (2003): Transfer for primary angioplasty versus immediate thrombolysis in acute myocardial infarction: a meta-analysis. Circulation $\underline{108}, 1809-14$

De Vreede JJ, Gorgels AP, Verstraaten GM, Vermeer F, Dassen WR, Wellens HJ (1991): Did prognosis after acute myocardial infarction change during the past 30 years? A meta-analysis. J Am Coll Cardiol 18, 698-706

Fox KAA, Cokkinos DV, Deckers JW, Keil U, Maggioni AP, Steg PG (2000): The ENACT study: a pan-European survey of acute coronary syndromes. European network for acute coronary treatment. Eur Heart J 21, 1440-9 
Garvey JL, MacLeod BA, Sopko G, Hand MM; National Heart Attack Alert Program (NHAAP) Coordinating Committee; National Heart, Lung, and Blood Institute (NHLBI); National Institutes of Health (2006): Pre-hospital 12-lead electrocardiography programs: a call for implementation by emergency medical services systems providing advanced life support--National Heart Attack Alert Program (NHAAP) Coordinating Committee; National Heart, Lung, and Blood Institute (NHLBI); National Institutes of Health. J Am Coll Cardiol $\underline{47}, 485-91$

Gibbons J, Holmes DR, Reeder CS, Bailey KR, Hopfenspirger MR, Gersh BJ (1993): Immediate angioplasty compares with the administration of a thrombolytic agent followed by conservative treatment for myocardial infarction. The Mayo Coronary Care Unit and Catheterization Laboratory Group. N Engl J Med $\underline{328}$, 685-91

Grines CL, Browne KF, Marco J, Rothbaum D, Stone GW, O'Keefe J, Overlie P, Donohue B, Chelliah N, Timmis GC, et al for the Primary Angioplasty in Myocardial Infarction Study Group (1993): A comparison of immediate angioplasty with thrombolytic therapy for myocardial infarction. N Engl J Med 328, 673-9

Hamm CW, Arntz HR, Bode C, Giannitsis E, Katus H, Levenson B, Nordt Th, Neumann FJ, Tebbe U, Zahn R (2004): Leitlinien: Akutes Koronarsyndrom (ACS), Teil 2: Akutes Koronarsyndrom mit ST-Hebung. Z Kardiol 93, 324-41

Henry TD, Sharkey SW, Burke MN, Chavez IJ, Graham KJ, Henry CR, Lips DL, Madison JD, Menssen KM, Mooney MR (2007): A regional system to provide timely access to percutaneous coronary intervention for ST-elevation myocardial infarction. Circulation $\underline{116}$, $721-8$

Hochman JS, Sleeper LA, Webb JG, Sanborn TA, White HD, Talley JD, Buller CE, Jacobs AK, Slater JN, Col J (1999): Early revascularization in acute myocardial infarction complicated by cardiogenic shock. SHOCK Investigators. Should we emergently revascularize occluded coronaries for cardiogenic shock. N Engl J Med 341, 625-635 
Keeley EC, Boura JA, Grines CL (2003): Primary angioplasty versus intravenous thrombolytic therapy for acute myocardial infarction: a quantative review of 23 randomised trials. Lancet $\underline{361}, 13-20$

Khot UN, Johnson ML, Ramsey C, Khot MB, Todd R, Shaikh SR, Berg WJ (2007): Emergency department physician activation of the catheterization laboratory and immediate transfer to an immediately available catheterization laboratory reduce door-to-balloon time in ST-elevation myocardial infarction. Circulation $\underline{116}, 67-76$

Le May MR, So DY, Dionne R, Glover CA, Froeschl MP, Wells GA, Davies RF, Sherrard HL, Maloney J, Marquis JF (2008): A citywide protocol for primary PCI in ST-segment elevation myocardial infarction. N Engl J Med $\underline{358}, 231-40$

Marciniak TA, Ellerbeck EF, Radford MJ, Kresowik TF, Gold JA, Krumholz HM, Kiefe CI, Allman RM, Vogel RA, Jencks SF (1998). Improving the quality of care for Medicare patients with acute myocardial infarction: results from the Cooperative Cardiovasular Project. JAMA $\underline{279}, 1351-7$

Maier SK, Ahlersmann D, Jung J, Fleischmann C, Werner GS, Maier LS, Olbrich HG, Hilgers R, Keating FK, Scholz KH (2009): Reduced Contact-to-Balloon Time in ST Elevation Myocardial Infarction as a Consequence of Formalized Data Analysis and Feedback: OneYear Results of the German Multicenter FITT-STEMI-Trial. Circulation 120, Nov 2009, 869

McNamara RL, Wang Y, Herrin J, Curtis JP, Bradley EH, Magid DJ, Peterson ED, Blaney M, Frederick PD, Krumholz HM; NRMI Investigators (2006 a): Effect of door-to-balloon time on mortality in patients with ST-segment elevation myocardial infarction. J Am Coll Cardiol $\underline{47}, 2180-6$

McNamara RL, Herrin J, Bradley EH, Portnay EL, Curtis JP, Wang Y, Magid DJ, Blaney M, Krumholz HM; NRMI Investigators (2006 b): Hospital improvement in time to reperfusion in patients with acute myocardial infarction, 1999 to 2002. J Am Coll Cardiol 47, 45-51 
Nadler DA: Feedback and organization development: using data-based methods. New York (NY): Addison-Wesley Publishing Company; 1977

Nallamothu BK, Bates ER, Herrin J, Wang Y, Bradley EH, Krumholz HM;NRMI Investigators (2005): Times to Treatment in transfer patients undergoing primary percutaneous coronary intervention in the United States, PhD; National Registry of Myocardial Infarction (NRMI)-3/4 analysis. Circulation 111, 761-7

Nallamothu BK, Bates ER, Wang Y, Bradley EH, Krumholz HM (2006): Driving times and distances to hospitals with percutaneous coronary intervention in the United States: implications for prehospital triage of patients with ST-elevation myocardial infarction. Circulation $\underline{113}, 1189-95$

Nunn CM, O'Neill WW, Rothbaum D, Stone GW, O'Keefe J, Overlie P, Donohue B, Grines L, Browne KF, Vlietstra RE (1999): Long-term outcome after primary angioplasty: a report from the primary angioplasty in myocardial infarction (PAMI-I) trial. J Am Coll Cardiol 33, 640-6

Rathore SS, Curtis JP, Chen J, Wang Y, Nallamothu BK, Epstein AJ, Krumholz HM; National Cardiovascular Data Registry (2009): Association of door-to-balloon time and mortality in patients admitted to hospital with ST elevation myocardial infarction: national cohort study. BMJ $\underline{338}:$ b1807

Reimer KA, Lowe JE, Rasmussen MM, Henning RG (1977): The wavefront phenomenon of ischemic cell death. 1. Myocardial infarct size vs. duration of coronary occlusion in dogs. Circulation $\underline{56}, 786-94$

Rustige J, Schiele R, Burczyk U, Koch A, Gottwik M, Neuhaus KL, Tebbe U, Uebis R, Senges J (1997): The 60 minutes myocardial infarction project. Treatment and clinical outcome of patients with acute myocardial infarction in Germany. Eur Heart J $\underline{18}$, 1438-46 
Schneider H, Ince H, Rehders T, Körber T, Weber F, Kische S, Chatterjee T, Nienaber CA; Drip\&Ship-Netzwerk (District of Rostock Infarct Project \& Shipping Patients) (2007):

Behandlung des akuten ST-Hebungsinfarkts in Netzwerkstrukturen ("Drip \& Ship Network Rostock"). Herz $\underline{32}, 635-40$

Scholz KH, Hilgers R, Ahlersmann D, Duwald H, Nitsche R, von Knobelsdorff G, Volger B, Möller K, Keating FK (2008): Contact-to-balloon time and door-to-balloon time after initiation of a formalized data feedback in patients with acute ST-elevation myocardial infarction. Am J Cardiol 101, 46-52

Soumerai SB, McLaughlin TJ, Gurwitz JH, Guadagnoli E, Hauptman PJ, Borbas C, Morris N, McLaughlin B, Gao X, Willison DJ (1998): Effect of local medical opinion leaders on quality of care for acute myocardial infarction: a randomized controlled trial. JAMA $\underline{279}, 1358-63$

Statistisches Bundesamt, Pressemitteilung vom 15. September 2006

Steg PG, Bonnefoy E, Chaubaud S, Lapostolle F, Dubien PY, Cristofini P, Leizorovicz A, Touboul P (2003): Impact of time to treatment on mortality after prehospital fibrinolysis or primary angioplasty. Circulation $\underline{108}, 2851-6$

The GRACE Investigators (2001): Rationale and design of the GRACE (Global Registry of Acute Coronary Events) Project: a multinational registry of patients hospitalized with acute coronary syndromes. Am Heart J 141(2), 190-9

The Joint European Society of Cardiology/American College of Cardiology Committee (2000): Myocardial infarction redefined - a consensus document of the joint European society of cardiology/american college of cardiology for the redefinition of myocardial infarction. Eur Heart J 21, 1502-13

Ting HH, Raveendran G, Lennon RJ, Long KH, Singh M, Wood DL, Gersh BJ, Rihal CS, Holmes DR Jr (2006): A total of 1,007 percutaneous coronary interventions without onsite cardiac surgery: acute and long-term outcomes. J Am Coll Cardiol 47, 1713-21 
Ting HH, Rihal CS, Gersh BJ, Haro LH, Bjerke CM, Lennon RJ, Lim CC, Bresnahan JF, Jaffe AS, Holmes DR (2007): Regional systems of care to optimize timeliness of reperfusion therapy for ST-elevation myocardial infarction: the Mayo Clinic STEMI Protocol. Circulation $\underline{116}, 729-36$

Ting HH, Krumholz HM, Bradley EH, Cone DC, Curtis JP, Drew BJ, Field JM, French WJ, Gibler WB, Goff DC (2008): Implementation and integration of prehospital ECGs into systems of care for acute coronary syndrome: a scientific statement from the American Heart Association Interdisciplinary Council on Quality of Care and Outcomes Research, Emergency Cardiovascular Care Committee, Council on Cardiovascular Nursing, and Council on Clinical Cardiology. Circulation 118, 1066-79

Tunstall-Pedoe H, Kuulasmaa K, Mahonen M, Tolonen H, Ruokokoski E, Amouyel P (1999): Contribution of trends in survival and coronary-event rates to changes in coronary heart disease mortality: 10-year results from 37 WHO MONICA project populations. Monitoring trends and determinant in cardiovascular disease. Lancet $\underline{353}$, 1547-57

Urban P, Stauffer JC, Bleed D, Khatchatrian N, Amann W, Bertel O, van den Brand M, Danchin N, Kaufmann U, Meier B (1999): A randomized evaluation of early revascularization to treat shock complicating acute myocardial infarction. The (Swiss) Multicenter Trial of Angioplasty for Shock-(S)MASH. Eur Heart J 20, 1030-8

Van de Werf F, Ardissino D, Betriu A, Cokkinos D, Falk E, Fox K, Julian D, Lengyel M, Neumann F-J, Ruzyllo W (2003): Management of acute myocardial infarction in patients presenting with ST-segment elevation. Task Force of the ESC. Eur Heart J 24, 28-66

Vakili BA, Kaplan R, Brown DL (2001): Volume-outcome relation for physicians and hospitals performing angioplasty for acute myocardial infarction in New York state. Circulation 104, 2171-6 
Wagner S, Schneider S, Schiele R, Fischer F, Dehn H, Grube R, Becker G, Baumgärtel B, Altmann E, Senges J (1999): Akuter Myokardinfarkt in Deutschland im Zeitraum zwischen 1996 und 1998: Therapie und hospitaler Verlauf. Ergebnisse des Myokardinfarktregisters (MIR) in Deutschland. Z Kardiol $\underline{88}, 857-67$

Wharton TP Jr, Grines LL, Turco MA, Johnston JD, Souther J, Lew DC, Shaikh AZ, Bilnoski W, Singhi SK, Atay AE (2004): Primary angioplasty in acute myocardial infarction at hospitals with no surgery on-site (the PAMI-No SOS study) versus transfer to surgical centers for primary angioplasty. J Am Coll Cardiol 43, 1951-3

Widimsky P, Budesinsky T, Vorac D, Groch L, Zelizko M, Aschermann M, Branny M, St'asek J, Formanek P; 'PRAGUE’ Study Group Investigators (2003): Long distance transport for primary angioplasty vs. immediate Thrombolysis in acute myocardial infarction. Final results of the randomized national multicentre trial-PRAGUE-2. Eur Heart $\mathbf{J} \underline{24}, 94-104$

Wienbergen H, Gitt AK, Schiele R, Juenger C, Heer T, Meisenzahl C, Limbourg P, Bossaller C, Senges J; MITRA PLUS Study Group (2003): Comparison of clinical benefits of clopidogrel therapy in patients with acute coronary syndromes taking atorvastatin versus other statin therapies. Am J Cardiol $\underline{92}, 285-8$

Wienbergen H, Gitt AK, Senges J (2005): Aktuelle Umsetzung der leitlinienkonformen Therapie des akuten ST-Hebungsinfarkts in Deutschland. Herz $\underline{30}$, 700-3

Zahn R, Schuster S, Schiele R, Seidl K, Voigtländer T, Meyer J, Hauptmann KE, Gottwik M, Berg G, Kunz T (1999): Comparison of primary angioplasty with conservative therapy in patients with acute myocardial infarction and contraindications for thrombolytic therapy. Cathet Cardiovasc Intervent $\underline{46}, 127-33$

Zahn R, Schiele R, Schneider S, Gitt AK, Wienbergen H, Seidl K, Bossaller C, Büttner HJ, Gottwik M, Altmann E (2000): Decreasing hospital mortality between 1994 and 1998 in patients with acute myocardial infarction treated with primary angioplasty but not in patients treated with intravenous thrombolysis. 
Results from the pooled data of the Maximal Individual Therapy in Acute Myocardial Infarction (MITRA) Registry and the Myocardial Infarction Registry (MIR). J Am Coll Cardiol $\underline{36}$, 2064-71

Zahn R, Schiele R, Gitt AK, Schneider S, Seidl K, Voigtländer T, Gottwik M, Altmann E, Gieseler U, Rosahl W; Maximal Individual Therapy in Acute Myocardial Infarction (MITRA) Study Group; Myocardial Infarction Registry Study Group (2001): Impact of prehospital delay on mortality in patients with acute myocardial infarction treated with primary angioplasty and intravenous thrombolysis. Am Heart J 142, 105-11

Zahn R, Vogt A, Zeymer U, Gitt AK, Seidl K, Gottwik M, Weber MA, Niederer W, Mödl B, Engel HJ; Arbeitsgemeinschaft Leitender Kardiologischer Krankenhausärzte (2006): Inhospital time to treatment of patients with acute ST elevation myocardial infarction treated with primary angioplasty: determinants and outcome. Results from the registry of percutaneous coronary interventions in acute myocardial infarction of the Arbeitsgemeinschaft Leitender Kardiologischer Krankenhausarzte. Heart 91, 1041-6

Zeymer U: PREMIER (Prähospitales Myokardinfarktregister). Jahrestagung der Deutschen Gesellschaft für Kardiologie 2005, H1 171

Zeymer U, Gitt A, Senges J (2005): Akuter ST-Strecken-Hebungs-Myokardinfakt. Aktuelle Versorgungssituation der Patienten in Deutschland. Herz 30, 241-3

Zijlstra F, de Boer MJ, Hoorntje JC, Reiffers S, Reiber JH, Suryapranata H (1993): A comparison of immediate coronary angioplasty with intravenous streptokinase in acute myocardial infarction. N Engl J Med $\underline{328}, 680-4$

Zijlstra F, Hoorntje JC, de Boer MJ, Reiffers S, Miedema K, Ottervanger JP, van 't Hof AW, Suryapranata H (1999): Long-term benefit of primary angioplasty as compares with thrombolytic therapy for acute myocardial infarction. N Engl J Med 341, 1413-19

Zijlstra F (2003): Angioplasty vs. thrombolysis for acute myocardial infarction: a quantative overview of the effects of interhospital transportation. Eur Heart J 24, 21-3 


\section{Veröffentlichungen}

S.K. Maier, D. Ahlersmann, J. Jung, C. Fleischmann, G.S. Werner, L.S. Maier, H.-G. Olbrich, R. Hilgers, F.K. Keating, K.H. Scholz:

Reduced Contact-to-Balloon Time in ST Elevation Myocardial Infarction as a Consequence of Formalized Data Analysis and Feedback: One-Year Results of the German Multicenter FITT-STEMI-Trial.

Circulation, Vol 120, Suppl.2, November 3, 2009, Seite 869

D. Ahlersmann, K. Möller, H. Duwald, R. Nitsche, R. Hilgers, G. von Knobelsdorff, F.

K. Keating, K. H. Scholz:

Deutliche Verkürzung der Reperfusionszeiten durch systematische Umgehung der

Notaufnahme bei Patienten mit akutem ST-Streckenhebungsinfarkt.

Clin Res Cardiol 98-Suppl 1, April 2009+V1601

D. Ahlersmann, C. Fleischmann, R. Hilgers, J. Jung, L. S. Maier, S. K. G. Maier, H.-G. Olbrich, K. H. Scholz, G. S. Werner:

Systematische Ergebnisrückkopplung in der Herzinfarktversorgung - Konzeption und Umsetzung der multizentrischen FITT-STEMI-Studie.

Clin Res Cardiol 98-Suppl 1, April 2009+P163

\section{K.H. Scholz, D. Ahlersmann, G. von Knobelsdorff:}

Überleben durch Dokumentation und Kommunikation-Versorgung des Akuten

Koronarsyndroms.

Notfall und Intensivmedizin 1/2009;8-11

\section{K.H. Scholz, D. Ahlersmann:}

Mehr Leben retten.

Niedersächsisches Ärzteblatt 1/2009;19-23 
D. Ahlersmann, K.H. Scholz, H. Duwald, R. Nitsche, G. von Knobelsdorff, B. Volger, K. Möller, R. Hilgers, F.K. Keating:

Bypassing the emergency room after pre-hospital ECG transmission as a major contributor to reduced door-to-balloon times in patients with acute ST-elevation myocardial infarctions.

J Am Coll Cardiol 2008; 51;Suppl A;1017-55

D. Ahlersmann, K. Möller, R. Hilgers, H. Duwald, R. Nitsche, G. von Knobelsdorff, K. H. Scholz:

Bypassing der Notaufnahme führt zu erheblicher Verkürzung der Reperfusionszeiten bei akutem ST-Streckenhebungsinfarkt.

Clin Res. Cardiol 97-Suppl 1 (2008)+V1005

D. Ahlersmann, R. Hilgers, H. Duwald, R. Nitsche, G. von Knobelsdorff, K. Möller, K. H. Scholz:

Hausarztkontakt führt zu markanter Verlängerung der Prähospitalzeit bei akutem STStreckenhebungsinfarkt.

Clin Res Cardiol 97-Suppl 1(2008)+V1004

K.H. Scholz, G. von Knobelsdorff, D. Ahlersmann, F.K. Keating, J. Jung, G.S. Werner, R. Nitsche, H. Duwald, R. Hilgers:

Prozessentwicklung in der Herzinfarktversorgung: Netzwerkbildung, Telemetrie und standardisiertes Qualitätsmanagement mit systematischer Ergebnis-Rückkopplung. Herz 2008;33:102-9

\section{K.H. Scholz, D. Ahlersmann, G. von Knobelsdorff:}

ACS-Diagnostik und präklinische Therapie - Systematisches Qualitätsmanagement mit enger Einbindung des Rettungssystems führt zu besserer Versorgung.

18. Internationales Symposium Intensivmedizin und Intensivpflege Journal für Anästhesie und Intensivbehandlung 1/2008 
K.H. Scholz, R. Hilgers, D. Ahlersmann, H. Duwald, R. Nitsche, G. von Knobelsdorff, B. Volger, K. Möller, F.K. Keating:

Contact-to-Balloon Time and Door-to-Balloon Time After Initiation of a Formalized Data Feedback in Patients With Acute ST-Elevation Myocardial Infarction.

Am J Cardiol 2008;101:46-52. E-pub: 19.Nov.2007

\section{F.K. Keating, D. Ahlersmann, R. Hilgers, H. Duwald, R. Nitsche,} G. von Knobelsdorff, K. Moeller, K.H. Scholz:

Reduced door-to-balloon time in ST- elevation myocardial infarction as a consequence of formalized data analysis and feedback.

Circulation 2007;116:II 431-2

D. Ahlersmann, K. Möller, R. Nitsche, H. Duwald, G. von Knobelsdorff, R. Hilgers, F. Keating, K. H. Scholz:

Marked reduction in contact-to-balloon time and door-to-balloon time as a consequence of formalized data feedback in patients with acute myocardial infarction.

Eur Heart J (2007); 28 (Suppl.): 824

D. Ahlersmann, G. von Knobelsdorff, R. Hilgers, R. Nitsche, H. Duwald, K. Möller, K. H. Scholz:

Schlüsselrolle des prähospitalen Funk- EKGs zur Reduktion der Reperfusionszeiten bei der Therapie des akuten St- Streckenhebungsinfarktes in einem Herzinfarktnetz in ländlicher Region.

Clin Res Cardiol 96: Suppl. (2007) P1199

D. Ahlersmann, G. von Knobelsdorff, R. Hilgers, R. Nitsche, H. Duwald, K. Möller, K. H. Scholz:

Herzinfarktnetz in ländlicher Region: Ein Projekt zur Qualitätssicherung und zur kontinuierlichen Qualitätsverbesserung.

Clin Res Cardiol 96: Suppl. (2007) V1908 


\section{Danksagung}

Mein besonderer Dank gilt Herrn Prof. Dr. med. K.H. Scholz für die Überlassung des interessanten Themas und die Möglichkeit, diese Arbeit in der Abteilung Kardiologie des St. Bernward Krankenhauses Hildesheim durchführen zu können. Sein großes Engagement und stete Motivation waren für mich von großer Bedeutung. Ohne seine geduldige Durchsicht und fachkundigen Anregungen wäre die Niederschrift der Arbeit in dieser Form nicht möglich gewesen.

Prof. Dr. med. R. Hilgers danke ich für die geduldige Unterstützung bei der statistischen Auswertung und die vielen wertvollen Anregungen.

Hr. A. Radtke möchte ich für die freundliche Einweisung und Zusammenarbeit bei den verwendeten erweiterten Datenverarbeitungsprogrammen danken.

Allen Beteiligten des Herzinfarknetzes Hildesheim-Leinebergland (Interventionskardiologen, Personal des Herzkatheterlabors und der Notaufnahme, Rettungsdienst-System, Personal der Nicht-Interventionskliniken) möchte ich für die freundliche und kooperative Zusammenarbeit bei der Datenerfassung sowie für das eingebrachte Engagement bei den FeedbackVeranstaltungen danken. 


\section{Lebenslauf}

Ich wurde als Tochter von Monika und Hermann Ahlersmann am 09.04.1975 in Georgmarienhütte geboren. Meine Kindheit und Jugend verbrachte ich in Melle bei Osnabrück. Dort absolvierte ich auch meine Schulzeit, welche ich 1994 mit dem Abitur am Gymnasium Melle abschloss. Bereits frühzeitig wurde im Rahmen von Pflegepraktika im örtlichen Krankenhaus in Melle mein Interesse für die Medizin geweckt.

Zum Wintersemester 1994/1995 erhielt ich einen Studienplatz an der Medizinischen Hochschule Hannover und absolvierte dort mein gesamtes Studium bis zum Abschluss mit dem Bestehen der Ärztlichen Prüfung am 16.05.2001. Während meines Medizinstudiums sammelte ich im Rahmen von mehreren Famulaturen im In- und Ausland (u.a. National Heart \& Lung Institute, Imperial College School of Medicine in London) Erfahrungen in ganz unterschiedlichen medizinischen Bereichen, wobei das Hauptinteresse stets der Inneren Medizin galt. Das Praktische Jahr begann ich in der Abteilung für Innere Medizin im Krankenhaus Großburgwedel (Lehrkrankenhaus der Medizinischen Hochschule Hannover). Im Anschluss absolvierte ich ein Anästhesie-Tertial im Spital Wil (Lehrkrankenhaus der Universität Zürich) und schloss das Praktische Jahr mit dem chirurgischen Tertial im RobertKoch Krankenhaus in Gehrden (Lehrkrankenhaus der Medizinischen Hochschule Hannover) $\mathrm{ab}$.

Nach Erlangung der vorläufigen Approbation trat ich am 01.06.2001 eine Stelle als Ärztin im Praktikum im Krankenhaus Großburgwedel an. Im Verlauf entwickelte sich ein zunehmendes Interesse für die Kardiologie, so dass ich nach Beendigung der ÄIP-Zeit und damit Erlangung der Approbation (11.12.2002) am 01.01.2003 die Arbeit als Assistenzärztin in der Abteilung für Kardiologie im Krankenhaus Peine aufnahm. Um ein breiteres kardiologisches Spektrum unter Einschluss der invasiven Kardiologie kennenzulernen, wechselte ich am 01.07.2003 in die Abteilung für Kardiologie und Intensivmedizin des St. Bernward Krankenhauses in Hildesheim, in der ich bis heute als Assistenzärztin tätig bin. 